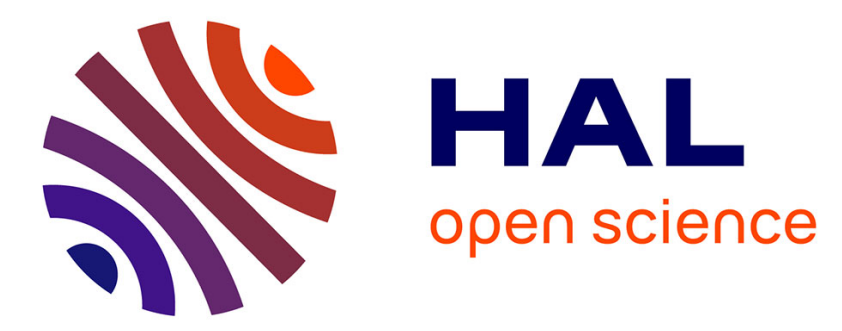

\title{
Experimenting with Mixtures of Water Ice and Dust as Analogues for Icy Planetary Material
}

Antoine Pommerol, B. Jost, O. Poch, Z. Yoldi, Y. Brouet, A. Gracia-Berná, R. Cerubini, A. Galli, P. Wurz, B. Gundlach, et al.

\section{- To cite this version:}

Antoine Pommerol, B. Jost, O. Poch, Z. Yoldi, Y. Brouet, et al.. Experimenting with Mixtures of Water Ice and Dust as Analogues for Icy Planetary Material. Space Science Reviews, 2019, 215 (5), pp.art. 37. 10.1007/s11214-019-0603-0 . insu-02181403

\section{HAL Id: insu-02181403 https://hal-insu.archives-ouvertes.fr/insu-02181403}

Submitted on 15 Jan 2022

HAL is a multi-disciplinary open access archive for the deposit and dissemination of scientific research documents, whether they are published or not. The documents may come from teaching and research institutions in France or abroad, or from public or private research centers.
L'archive ouverte pluridisciplinaire HAL, est destinée au dépôt et à la diffusion de documents scientifiques de niveau recherche, publiés ou non, émanant des établissements d'enseignement et de recherche français ou étrangers, des laboratoires publics ou privés. 


\title{
Experimenting with mixtures of water ice and dust as analogues for icy planetary material. \\ Recipes from the Ice Laboratory at the University of Bern.
}

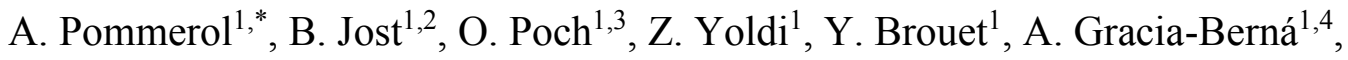

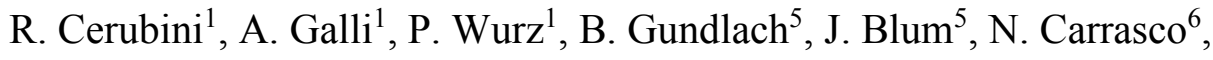 C. Szopa ${ }^{6}$, N. Thomas ${ }^{1}$

\begin{abstract}
${ }^{1}$ Physikalisches Institut and NCCR PlanetS, Universität Bern, Switzerland.
${ }^{2}$ Now at Jet Propulsion Laboratory, Pasadena CA, USA.

${ }^{3}$ Now at Univ. Grenoble Alpes, CNRS, IPAG, Grenoble, France.

${ }^{4}$ Now at Boeing Research \& Technology Europe, Madrid, Spain.

${ }^{5}$ Institut für Geophysik und extraterrestrische Physik, TU Braunschweig, Germany.

${ }^{6}$ Université Versailles St-Quentin, Sorbonne Universités, UPMC Univ. Paris 06, CNRS/INSU, LATMOS-IPSL, Guyancourt, France.
\end{abstract}

\author{
*To whom correspondence should be addressed: \\ Antoine Pommerol \\ ORCID ID: 0000-0002-9165-9243 \\ Physikalishes Institut, Universität Bern \\ Sidlerstrasse 5, CH-3012 Bern, Switzerland \\ antoine.pommerol@space.unibe.ch \\ +41316313998
}

First revision, submitted 21.12.2018

Keywords: Ice, Dust, Analogues, Photometry, Spectrometry 


\section{Abstract}

Due to its abundance and unique properties, water is a major actor in the formation and evolution of many planetary surfaces as well as a sensitive and reliable tracer of past geologic and climatic processes. Water ice is found in variable abundance at the surfaces of many Solar System objects, from the floor of permanently shadowed craters at the poles of Mercury to large fractions of the surfaces of several trans-Neptunian objects. With few exceptions, water is not found in pure form but associated to contaminants of various nature and concentration. These associations and the nature of the mixing and segregation processes that affect and control them are key for our understanding of some of the most important aspects of planetary evolution processes. The observation and characterization of water ice at the surface of Solar System objects is therefore among the primary scientific objectives of many space missions. The quantitative interpretation of remote sensing data in terms of surface composition and physical properties requires the use of complex physical models that rely on experimental data in two different ways. First, the models require as inputs the fundamental properties of the pure materials, such as the optical or dielectric constant. Second, the models can only be fully tested if their results are confronted to actual measurements performed on samples whose complexity comes close to the one encountered on natural planetary surfaces but which are nevertheless wellenough characterized to serve as reference. Such measurements are challenging as macroscopic ice-rich samples prepared as analogues of icy planetary surfaces tend to be unstable, the ice component being prone to metamorphism and phase change. The questions of the reproducibility of the samples and the relevance of the measurements are therefore critical. The Ice Laboratory at the University of Bern has been set up in 2010 to overcome some of these difficulties. We have developed protocols to prepare, store, handle and characterize various associations of ice with mineral and organics contaminants as analogues of different types of icy Solar System surfaces. The aims of this article are to present the context and background for our investigations, describe these protocols and associated hardware in a comprehensive way, provide quantitative characterization of the samples obtained using these protocols and summarize the main results obtained so far by experimenting with these samples. The current state and possible future evolutions of this project are then discussed in the context of the next generation of space missions to visit icy objects in the Solar System and longer term perspectives on future observations of protoplanetary discs and exoplanetary systems. 


\section{Introduction}

In the field of planetary remote sensing, laboratory experiments essentially serve two complementary purposes:

- First, they can provide the fundamental physical quantities of the materials that compose planetary surfaces, which are essential for their identification, characterization and the modelling of processes. For optical remote sensing, the most important quantities are the optical constants (Palik, 1991). Together with various macroscopic properties of the surface such as porosity and roughness, these quantities will determine how and how much light is scattered by the surface (Hapke, 1993). Such fundamental physical quantities are generally retrieved from well controlled experiments in which the analysed material is produced or prepared pure and with a simple geometry, for instance single crystals, parallel thin films or isolated single particles (Schmitt et al., 1998).

- Second, they can mitigate the lack of ground-truth for most actual planetary surfaces. Macroscopic analogue surfaces that reproduce some of the complexity of actual planetary surfaces can be used to test the performances of the models developed to invert physical quantities from the remote sensing data (Shepard and Helfenstein, 2007). When models fail or cannot be used, direct comparisons of laboratory data acquired on such samples with the actual remote sensing data are often the best way of interpreting these data.

Although crucial, mimicking actual planetary surfaces in the laboratory is a challenging task because of the many unconstrained properties and the effects of various environmental processes that are difficult to reproduce. Icy surfaces, which are widespread in the Solar System provide additional challenges as the volatile nature of the ice imposes strict and demanding experimental conditions to ensure of the reproducibility and representativeness of the experiments and their results.

This field of research was pioneered in the Soviet Union in the late seventies. Dobrovolsky and Kajmakow (1977) exposed a mixture of water ice and dust to a lowtemperature vacuum environment and witnessed the formation of a sublimated layer at the surface of the sample which was regularly affected by outbursts. Similar experiments were conducted at JPL in the eighties with various compositions of the dust component (Saunders et al., 1986; Storrs et al., 1988) from which the structure of the sublimated layer could be analysed in detail. The most comprehensive set of experiments in this field was then performed in Germany in the late 1980s to early 1990s, a program known as "KOSI" (Kometen Simulationen, Grün et al., 1991). A total of eleven experiments were conducted in a large simulation chamber with samples containing $\mathrm{H}_{2} \mathrm{O}$ and $\mathrm{CO}_{2}$ ices mixed intimately with a dust composed of dunite powder and black carbon. This program provided a wealth 
of information on the behaviour of ice/dust mixtures in a simulated space environment. It revealed for instance the importance of the vertical transport of water through the insulated layer and, under favourable circumstances, the formation of a crust enriched in recondensed water ice under the desiccated uppermost layer. Three attempts were made at characterizing quantitatively the change in the spectro-photometric properties of the samples (KOSI-3, 4 and 6) upon desiccation (Oehler and Neukum, 1991). The spectra recorded after the exposure of the samples to vacuum only show some residual spectral features of water ice but contamination of the samples by frost deposited from the laboratory atmosphere after the extraction of the samples from the chamber cannot be excluded. The absence of spectral features from the mineral dust is attributed to the strong absorption by the carbon particles. After the end of the KOSI program, additional experiments were conducted at the IWF in Graz using a smaller facility to investigate some particular points of high interest. The effect of the presence of organics on the properties of the sublimation residue was studied in details using paraffins, aliphatic hydrocarbons, as analogues for cometary organics (Kömle et al., 1996). Paraffins were found to act as a glue, cementing the mineral particles in the desiccated layer and strongly affecting its mechanical and thermal properties.

In parallel to the ambitious simulation experiments described previously, the spectrophotometric properties of some associations between water ice and dust have been investigated and reported in a number of publications. Clark and Lucey (1984) have published visible and near-infrared reflectance spectra of water ice with three different types of inclusions: kaolinite (clay mineral), a Hawaiian soil rich in iron oxide and charcoal. The samples were produced by preparing a suspension of particles into liquid water and then freezing this suspension in a sample holder cooled by liquid nitrogen. Roush et al. (1990) reused the same technique with additional minerals but ultimately crushed the resulting solid samples and sieved the powder to isolate particles approximately $100 \mu \mathrm{m}$ in diameter. Stephens and Gustafson (1991) measured the visible bidirectional reflectance of mixtures of water ice, colloidal silica and carbon for a few different scattering geometries and observed the effect of the sublimation of the ice on the bidirectional reflectance of the sample. The sample preparation protocol was similar to the one used by Clark and Lucey (1984), starting from a suspension of the silica and carbon particles into liquid water.

Many more results can be found in the literature published by the terrestrial remote sensing community including detailed characterizations of the spectral Bidirectional Reflectance Distribution Function (BRDF) of snows with variable degrees of metamorphism (see for instance Hudson et al. (2006); Kaasalainen et al. (2006); Dumont et al., 2010). With a few exceptions, mostly in the context of Mars, the reflectance properties of terrestrial snow, sea ice or glaciers are however of very little relevance for 
other Solar System surfaces. Surface processing of most airless icy bodies differs vastly from surface processes on Earth, where liquid water is involved. This precludes a direct application of measurements on natural terrestrial samples and surfaces to most of other Solar System surfaces. For instance, phase curves of various icy moons have been compared to phase curves of terrestrial snow by Verbiscer and Veverka (1990). The strong backscattering behaviour observed on the icy moons is not found in any of the terrestrial snow and frost samples measured. It has however been reproduced when measuring the reflectance of $\mu \mathrm{m}$-sized spherical particles of pure water ice (Jost et al., 2013) initially developed as an analogue for cometary ice (Gundlach et al., 2011b).

With the development of the Ice Laboratory initiated in 2010 at the University of Bern (Pommerol et al., 2011), we aim at filling a gap in the literature on the measured properties of samples made of water ice mixed with dust. The main focus of the laboratory is the measurement of spectro-photometric properties of the samples in the visible and nearinfrared spectral range. These data should serve to interpret datasets collected by various optical remote sensing instruments on-board space missions, either by direct comparison of measured data or through the testing of physical models. In addition, we want to distribute the samples so that they can be characterized and measured with different techniques, progressively assembling a comprehensive library of data on a suite of wellcharacterized reference samples.

This manuscript serves two purposes. It provides recipes by detailing the procedures and techniques we have developed over the years to produce well calibrated icy samples. It also explains the motivations behind these developments by describing the general scientific context for the experiments we perform and by summarizing the most important results obtained so far. Section 2 provides background and context for the investigations pursued through this project. The importance of water ice in the formation and evolution of planetary surfaces is explained and the remote sensing techniques used to detect and characterize water ice on these surfaces are briefly described. The remote sensing observations most relevant for our experimental work are then summarized and a description of the objectives and history of our project concludes this chapter. Section 3 details the procedures used to produce and characterize the icy samples. In Section 4, we summarize the results obtained with the ice/dust mixtures and how they apply to various cases of Solar System surfaces. These results have been published in various articles over seven years and this chapter acts as a sort of table of contents for these results. Finally, Section 5 discusses the current status of our project including the main achievements and current limitations, and lists ideas for future improvements and new developments. 


\section{Background and context}

\subsection{The importance of water for Solar System formation and evolution}

Constituted of the first and third most abundant elements in the Universe, the water molecule is widespread in a variety of astrophysical environments (Hanslmeier, 2011). Because of their shape and polarity, $\mathrm{H}_{2} \mathrm{O}$ molecules can establish numerous hydrogen bonds between them, which result in unique physical and chemical properties (see Linstrom and Mallard, 2005 for reference values). The phase diagram of water (Wagner and Pruß, 2002) for instance, shows a number of particularities such as the very high temperature of its boiling point, $373.16 \mathrm{~K}$ at atmospheric pressure, compared to other molecules of similar weight. These strong hydrogen bonds are also responsible for the very high specific heat capacity of water in both solid and liquid states. Liquid water also shows a very high surface tension and capillarity. These properties strongly influence the behaviours of water, in particular its diffusion through porous media and its adhesion to various substrates, which in turn has a strong effect on the ability of water to interact chemically with other substances or act as a medium for chemical reactions and transport of substances (Snoeyink and Jenkins, 1980). Water vapour can be adsorbed in large quantities on a variety of solid surfaces, with a very high affinity for solids presenting hydroxyl groups at their surface. Thin films of adsorbed water have properties that resemble the ones of liquid water and in particular can dissolve and transport ions, enabling chemical reactions (Möhlmann, 2005). The phase transitions are affected by the presence of impurities. Salty solutions at the eutectic point can be liquid at temperatures much below the triple point of water. The rate of sublimation of the ice, i.e. the direct transition from solid to gas phase in vacuum or at low temperature, is observed to be affected by the presence of dust (Gundlach et al., 2011a; Kossacki and Markiewicz, 2013). A comprehensive review of available data on the sublimation of water ice on a broad range of temperatures is provided by Fray and Schmitt (2009).

\subsubsection{The role of water in Solar System formation}

Segregation and mixing of refractory and volatile elements over various timescales during the formation and evolution of planetary systems are among the most important processes that influence their currently observed characteristics. The long-term evolution of planetary systems can only be studied by models and simulations, supported and constrained by observations of the Solar System as well as, for the last twenty years, observations of extrasolar planets and planetary systems. The very early phases in the formation of planetary system can also be apprehended by astronomical observations of 
protoplanetary disks. Most of these observations in the case of the Solar System and all of them in the case of extrasolar planets and disks, can only be made from a distance, using remote sensing techniques from the surface of the Earth or from space probes and observatories. The inventory and characterization of water in the current Solar System have essentially been performed by remote sensing instruments on-board a multitude of spacecraft orbiting or flying by all planets of the Solar System as well as many minor planets and small bodies (see Section 2.2.2). The analyses of various classes of meteorites complements remote sensing observations by providing key elements on the timing of the Solar System formation and the origin of volatiles found in the inner Solar System.

Water represents about half of the total mass of all condensable species in the Solar System (Lodders, 2003). Yet, the inner Solar System is essentially dry, with water accounting for a maximum of $0.1 \%$ only of the total mass of the Earth (Marty, 2012; Okuchi, 1997) or Mars (Kurokawa et al., 2014) whereas it is very abundant in the outer Solar System in the form of ice, accounting for up to $75 \%$ of the mass of icy moons of Saturn (Anderson and Schubert, 2007). The boundary between the water-poor inner Solar System and the water-rich outer Solar System is located within the asteroid belt as attested by the observation of hydrated asteroids in the outer belt (Fornasier et al., 2014). This limit corresponds to the condensation line of water in the protoplanetary disk, which gave birth to the Solar System. It is referred to as the "ice-line" or "snowline" in the literature. Most models of disk evolution, however, predict that the snowline rapidly moves inwards as the disk cools down, long before the gas is dissipated. Therefore, the low amount of water in the Earth, Mars and most of the asteroid belt is difficult to explain.

Morbidelli et al. (2016) propose a solution to this apparent paradox. They first calculate that the radial motion of the gas towards the star is faster than the inwards motion of the snowline, effectively preventing direct condensation in the inner Solar System. Therefore, water can only be brought into the inner Solar System by the inwards radial drift of icy solid particles condensed at larger heliocentric distances. This process will however be inhibited by filtering from the proto-Jupiter, as soon as it reaches a mass of 20 Earth masses. Morbidelli et al. (2016) propose that this occurred when the snowline was at a heliocentric distance of $3 \mathrm{AU}$, thus efficiently preventing the hydration of all bodies closer to the Sun. Sato et al. (2015) propose an alternative scenario involving a rapid depletion of icy pebbles before the snowline starts migrating across the inner Solar System. This scenario is consistent with some of their simulations in the case of a rather small disk. They conclude that both scenarios are equally probable and that future studies and observations will be required to favour one or another. 
The rapid growth of solid agglomerates in a size range of a few centimetres up to one meter, referred to as "pebbles" (Lambrechts and Johansen, 2012) and its consequences for the early evolution of the outer Solar System have become very popular topics in the literature, being invoked in explanations to some of the outstanding issues and paradoxes in models of disk evolution and planetary systems formation (Lambrechts et al., 2014). In particular, this "pebble accretion scenario" provides an explanation for the formation of gas giants by enabling the rapid accretion of large cores before the gas has dissipated from the disk (Lambrechts and Johansen, 2014; Levison et al., 2015a). As mentioned in the previous paragraphs, it is also involved in plausible scenarios that can explain the depletion of water in the inner Solar System. Water, in the form of ice, plays a key role in the pebble accretion scenario, in two ways:

- First, the growth of the pebbles by accretion of smaller agglomerates of dust and ice can be strongly influenced by the "stickiness" of water, which is a direct consequence of the peculiar properties of the water molecule (Section 2.1). This has been studied experimentally by Gundlach and Blum (2015) who found a threshold velocity for micrometre-size water ice particle ten times higher than for particles of the same size and shape made of silica. Therefore, the rate of growth of the pebbles, a key parameter in the models, will be influenced by the amount and properties of the ice.

- Second, the water ice within the pebbles will strongly affect their evolution as they drift inwards and cross the ice-line. Some scenarios invoke the disruption of the larger pebbles in smaller particles as a consequence of ice sublimation to explain the small size of the terrestrial planets (Levison et al., 2015b). The fate of a pebble upon sublimation of the water ice remains however a completely open question and is probably highly dependent on unconstrained parameters such as the bulk porosity of the particles, the size of the dust and ice agglomerates, and the way they are mixed. Experiments are certainly required to better assess the evolution of pebbles that cross the ice-line. Beyond experiments and models, the study of comets is probably the best opportunity to learn about the role of water ice during the early phases of the disk evolution as cometary nuclei are assumed to consist of relatively pristine icy material, at least in their interior, possibly preserving the structure of the icy pebbles.

\subsubsection{Evolutionary surface processes involving water ice}

Water in its solid state dominates the surfaces of most of solid bodies in the outer Solar System. Both liquid water and ice have played key roles in the evolution of the icy moons, often similar to the role played by the silicates dominant in the inner Solar System. 
Solid-state convection, ice diapirism, tectonism and cryo-volcanism have shaped the surfaces of the icy moons over large scales.

Sublimation and condensation of water ice at or near the surface of various bodies can strongly influence the evolution of their surface shape. Surface features and textures shaped by sublimation have been observed at the surfaces of terrestrial planets, comets, icy satellites and Pluto. On the two terrestrial planets with atmosphere and ice-covered surface areas, the Earth and Mars, the amount and nature of contaminants found in the ice deposits in polar areas are reliable tracers of past climate changes (Becerra et al., 2017). Water, both in liquid and solid phase, can also be a very powerful agent of erosion on planets with an atmosphere and an active water cycle. Glaciers for instance carve valleys of variable size and shape depending on the parameters of the flow (Hauber et al., 2011). On the surfaces of comets but also other larger icy objects, peculiar features such as penitents (Lliboutry, 1954), pinnacles (White et al., 2016), troughs, pits, suncups, circular and scalloped depressions (Lefort et al., 2010) are all interpreted to be shaped by the sublimation of ice, either in vacuum or in a dry atmosphere. At smaller scale, many bulk physical properties of the desiccated surface layer of these objects, such as density, porosity, tortuosity but also the mechanical and thermal properties that derive from those are controlled by the modalities of the sublimation of the ice.

The visible "activity" of comets is the most spectacular expression of ice/dust segregation processes at play in today's Solar System. The gas formed by the sublimation of the cometary ices, mostly $\mathrm{H}_{2} \mathrm{O}, \mathrm{CO}_{2}$ and $\mathrm{CO}$ escapes the surface with high velocity, dragging with it some of the cometary dust that was previously intimately mixed or embedded into the ice. Because of the low thermal conductivity of porous cometary material, ices of water and more volatile elements can survive in the interior of the nucleus even at very low heliocentric distances. As a result, mixing between dry material from the inner Solar System and volatile-rich material from the outer Solar System still occurs today, although at a much lower rate than when terrestrial planets form. Water ice and organics can be transported and deposited by this process through the entire inner Solar System, all the way to Mercury where they are observed to accumulate in polar shadowed craters (Section 2.2.2). Depending at which heliocentric distance and at which depth in the subsurface it occurs, the sublimation of the ice can produce geomorphological changes over very different timescales. Meters of material can be lost within a couple of months, strongly affecting the overall shape and surface features of the nucleus, sometimes leading to its complete disruption (Steckloff et al., 2015). In addition to dust, large blocks of material can be levitated and either escape the low gravity field of the comet or fall back at other places on the surface. 
At smaller scales and depths, surface chemistry involving water ice affects the composition of the surface. Chemistry in ice phase triggered by cosmic rays and far-UV radiation is a very important topic for the formation and evolution of organics in the interstellar medium (ISM) and is the subject of an abundant literature reporting on the results of laboratory experiments performed on thin films samples (Nuth et al., 2006; Öberg et al., 2009; Strazzulla et al., 2011). As soon as the accretion starts however, the importance of these processes will decrease as the energetic radiations cannot penetrate deep in the surfaces and chemistry will be limited to surficial layers. The effects of water ice bombardment by energetic particles and radiation can still affect the albedo and colour of surfaces in the outer Solar System by either physical or chemical processes.

Surface sputtering by ions of various energies is a major cause of erosion of the icy surfaces. In addition to sputtering, the incident ions are able to either cause damage in the crystalline lattice of the ice leading to amorphisation of the ice (Strazzulla et al., 1992), or on the contrary to induce crystallization and grain growth (Moore and Hudson, 1992) depending on their energy and the state of the ice. Either darkening and reddening of the ice or brightening have been observed as a result of ion irradiation (Sack et al., 1992). Ions as well as electrons and energetic photons can break bonds in the ice and create radicals, triggering chemical reactions between the ice and its contaminants and/or the implanted ion. Photolysis and radiolysis are the two processes at play, distinguished by their excitation energy, lower or higher than the ionization energy, respectively. Gaseous reaction products can be trapped in amorphous ice and later released during the crystallization of the ice. The formation of a crust of dark and red complex organics by these processes is thought to dominate the evolution of the visible surfaces of most of the small bodies in the outer Solar System (Brunetto et al., 2006).

Note that if the presence of salts in soils is often interpreted as indicative of a past presence of liquid water, there might sometimes be alternative possibilities. In particular, it has been shown in laboratory experiments that the formation of calcium carbonate is possible in the absence of condensed liquid water, by interaction between the gas and water adsorbed at the surface of the solid grains (Garenne et al., 2013). The "quasi-liquid" behaviour of adsorbed water has also been mentioned in a number of other studies for various processes, including erosional processes and habitability (Stoker et al., 2010).

Despite its tremendous importance and decades of research, the question of the orgin of water on terrestrial planets is still an open question. It is possible that inner rocky planets have formed essentially dry and the small amounts of volatiles present on some of them were probably brought later by impact from hydrated and/or icy objects formed in the outer Solar System (Albarède, 2009). This process is still active today, although at a much 
lower rate than earlier in the history of the Solar System, and explains the presence of ice in permanently shadowed areas at the surface of atmosphereless objects. Some results from the Rosetta mission place constraints on how much water could have been brought to Earth by Jupiter-family comets (Altwegg et al., 2015; Marty et al. 2017) and measurements of the $\mathrm{D} / \mathrm{H}$ ratios in comets from other families also point to a minor contribution from these objects to terrestrial water. Other reservoirs such as water from hydrated asteroids and protoplanets from the outer asteroid belt must therefore be considered. Although the amount of volatiles brought to the inner Solar System was small, its effects were major, triggering large scale transformations that changed the entire surfaces of the Earth and Mars and enabling the emergence of life on at least the first of these two planets.

\subsection{Remote sensing of water ice}

\subsubsection{Detection and characterization of water ice by remote sensing}

Mapping the current occurrence of water ice in the Solar System and characterizing its properties through remote sensing provides new insights into its role in various aspects of the formation and evolution of the Solar System described in Section 2.1. A number of remote sensing techniques (see Table 1) are regularly used on orbital missions to assess the presence of water at or below a planetary surface and characterize its state and properties. Most of them are based on the interaction of electromagnetic radiations with the regolith, spanning the whole spectrum from radio waves to gamma rays. Table 1 provides a summary of these different remote sensing techniques. Beside methods based on the interaction between an electromagnetic field and the surface materials, other techniques exist that can provide information on water in planetary regoliths. Neutron spectroscopy is a widely used passive or active technique to retrieve quantitative measures of the hydrogen content of the close subsurface. The technique is based on the emission of high-energy, or "epithermal" neutrons within the first meter of the regolith. These fast neutrons strongly interact with the nuclei of hydrogen atoms, losing their energy by thermalisation and producing "thermal neutrons". Therefore, the measured ratio between thermal and epithermal neutrons is proportional to the bulk hydrogen content in about the first meter below the surface. In addition, it is worth noting the possibility of producing compositional maps from surfaces by directly measuring actual atoms, ions or molecules sputtered from

planetary surfaces. Although this not properly speaking a remote sensing technique, as it is based on an actual measurement using a mass spectrometer, it can be used to map the composition of surfaces in the same way as electromagnetic methods and 
provide highly complementary information, especially in identifying minor species (Vorburger et al., 2015).

Understanding the physics of the interaction between the electromagnetic field and the particulate surface is thus a requirement to analyse these remote sensing datasets quantitatively. Some of the physics involved is common to most or all of these techniques but the scales of the relevant parameters are highly dependent on the wavelength. This is the case of the scattering that is an important physical process in both optical and radio domains. The size of the particles or structures that cause the scattering is proportional to the wavelength and will thus be of the order of micrometres in the case of optical methods and of centimetres to hundreds of metres in the case of radar techniques. Similar scaling factors also apply to the penetration depths, leading to a strong complementarity between different remote sensing techniques able to probe different depths below the surface. Our experimental activities (Section 4) have mostly concentrated on the visible and nearinfrared spectral range so far, but are now diversifying into other domains. VIS-NIR remote sensing is thus treated in more detail in this section whereas other techniques are addressed more succinctly.

\section{Water-electromagnetic field interactions}

One of the basic inputs for all scattering models are the optical constants, or complex spectral refractive indices of the components. In the optical domain, the optical constants of water ice (Warren and Brandt, 2008) are dominated by the fundamental, harmonic and combination modes of the vibrational transitions of the water molecule (Banwell, 1983). The water molecule itself interacts differently with the electromagnetic field depending on the spectral domain but shows interactions over the totality of the spectrum. In fact, because the interaction of water with electromagnetic radiation is so strong, the most useful spectral domains to study water by remote sensing are the ones where its absorption if the lowest. In the visible spectral range $(0.4-1 \mu \mathrm{m})$, the absorption of water is the lowest over the entire electromagnetic spectrum, but still not completely negligible, increasing from the blue (minimum at $418 \mathrm{~nm}$ for liquid water, Pope and Fry, 1997) to the red.

Except for the Earth, to which it gives its characteristic blue colour, this effect is not observed in the Solar System because of the long optical path length and the high purity required. The extremely low absorption in the visible range is however responsible for the high albedo of fine-grained water ice that often provides the best possibilities for its detection and mapping at high spatial resolution. Note however that the presence of water, either liquid or as ice in the interstices of a soil leads to a drastic reduction of the reflectance. 
Two different explanations are generally invoked to explain this observation: internal total reflection within the film of water that coats the grains (Angstrom, 1925) or increase of the transmission of light to the grains because of the intermediate refraction index of water between the one of the vacuum or air and the one of the grains (Twomey et al., 1986). Therefore, the presence of ice on a planetary surface can have totally opposite effects depending on how the ice is mixed with the other components of the surface.

Water displays much more specific signatures in the near-infrared range $(1-5 \mu \mathrm{m})$ with various absorption due to overtones and combinations of the fundamental vibration modes of the $\mathrm{H}_{2} \mathrm{O}$ molecule. The resulting absorption bands observed in the light reflected from planetary surfaces permit the distinction between water ice and other ices such as carbon dioxide or nitrogen, which is not possible in the visible range. The positions, strengths and shapes of the bands are also sensitive to the crystallinity of the ice, its temperature and the size of its grains. Near-infrared reflectance spectroscopy and hyperspectral imaging are therefore among the most useful techniques for the identification and characterization of water ice on planetary surfaces. This is true as well in the case of liquid water that displays even stronger absorption bands, at slightly different positions than the ice.

Very strong absorptions occur in the ultraviolet $(10-400 \mathrm{~nm})$ and mid-far infrared $(5 \mu \mathrm{m}-1 \mathrm{~mm})$, due to electronic transitions and fundamental vibrations modes of the water molecule, respectively. As the resulting absorption bands get saturated for even low amounts of water and as the light fluxes from either reflected solar light or thermal emission are low, these spectral domains are of limited interest for studying the surfaces or waterrich objects and near-infrared is systematically preferred. The situation is different if water is a minor constituent as the strong absorptions can permit its identification and quantification. Of particular interest for that purpose are the strong absorptions at $165 \mathrm{~nm}$ in the far ultraviolet and at $6 \mu \mathrm{m}$ in the mid-infrared.

The absorption of water decreases steadily as the wavelength increases from the far-infrared (hundreds of $\mu \mathrm{m}$ ) to the microwave (millimetre and centimetre waves) and the radio domain (meters to hundreds of metres waves). However, a strong contrast of dielectric constant develops between the liquid water and the solid ice. At radar wavelength, the real dielectric constant of pure liquid water is about 80 whereas the one of hexagonal ice is about 3 (Heggy et al., 2001; Heggy et al., 2012). The dielectric constant is also directly proportional to the porosity of the material and radar data can thus be used to assess the porosity of icy surfaces and subsurfaces. 


\section{Detection of ice in the visible and near-infrared}

Visible imaging is based on the collection of Solar light reflected from the surface of an object by an optical instrument. Except for very few particular cases, the reflection of the solar light by a particulate planetary surface is purely diffuse and the dominant physical process is scattering. The scattering process can be represented as a sum of surface and volume interactions between the light rays and the solid particles. At each interface between a grain and the ambient medium, vacuum or atmosphere, part of the light is reflected by the surface and part of it is refracted within the grain through which it can be partly or totally absorbed or transmitted. Surface reflection and refraction is controlled by the refraction index $\mathrm{n}$, whereas the absorption is quantified by the extinction index $\mathrm{k}$. Refraction and extinction index are the real and imaginary indices, respectively, of the optical constants, more generally referred to as dielectric constants in other regions of the electromagnetic spectrum.

This simple description of the scattering process is already sufficient to identify the main parameters that control the reflection of light and intuitively understand their role. Changing the size of the grains will mostly affect their surface to volume ratio, and thus the relative influence of the reflection and absorption of photons by the grains through changes in the mean optical path length of the photons between their entrance and their exit from the regolith. As a consequence, the intrinsic optical signatures of the material will be more strongly expressed if the grains are large than if the grains are small. The absorption index of water is very slightly increasing from the blue to the red region of the visible spectrum. This gives a blue colour to the light reflected by ice, which can only be perceived if the optical path length of the photons exceeds one meter, as can be the case in the pure and compact ice of terrestrial glaciers. Water ice with small grains such as snow or frost results in very short optical path lengths, which won't permit the expression of the absorption properties of water and will thus appear completely white.

Changing the incidence and the emission direction will also generally strongly affect the reflectance of the surface. One of the most important processes that control the dependence of reflectance on the bidirectional geometry is the "shadow hiding", which is also easy to apprehend intuitively. In a porous regolith with opaque or semi-transparent grains, the grains of the uppermost layer cast shadows on the deeper grains. The length of the shadows depends directly on the phase angle, the angle between the incidence and emission directions. In the case of an observation in the opposition geometry, for which the incidence and emission directions are the same, no shadows are visible and the measured reflectance is maximal. As the phase angle increases, more shadows are visible and the reflectance decreases. This effect is known as the Shadow Hiding Opposition Effect 
(SHOE) and is observed on nearly all planetary surfaces. Large-scale topography can have similar effects, by generating shadows at scales unresolved by the observation.

\section{The Hapke model}

The most widely used reflectance model in planetary sciences is the one developed over three decades by Hapke and described in a series of books and papers published between 1981 and 2012 (Hapke, 1981; Hapke and Wells, 1981; Hapke, 1984; Hapke, 1993; Hapke, 2002; Hapke, 2005; Hapke, 2008; Hapke, 2012). It relates the radiance scattered from a planetary surface to a series of parameters, generally referred to as the "Hapke parameters" (Table Hapke, Figure 1), that describe the scattering of the light by individual particles and, depending on the version, the macroscopic roughness of the surface and its bulk porosity. All of these parameters are included in a model based on the theory of radiative transfer, building upon extensive work by Chandrasekhar, (1950) to describe the scattering of light in a porous medium composed of independent solid particles in contact.

The solution used by Hapke is not exact but uses approximations to simplify the problem. Some approximations made in earlier versions of the model have later been replaced by either exact or more accurate solutions. This is in particular the case of the Ambartsumian-Chandrasekhar $\mathrm{H}$-functions, which are approximated in a more accurate way since the 2002 version of the model (Hapke, 2002). In the same paper, Hapke also introduced a more accurate representation of the multiple scattering terms. Previous versions of the model only considered isotropic scatterers for the multiple scattering terms whereas the single-scattering term was calculated exactly. This earlier version of the model is known as the Isotropic Multiple Scattering Approximation (IMSA). Although the new version is more exact, most users of the model are still using the IMSA version today, as it is much faster to calculate and as the differences between the IMSA and the new version are negligible for all surfaces except the very bright ones.

Indeed, each individual particle of matter scatters light in an anisotropic way. This has been modelled by Mie for the ideal case of spherical isolated particles with sizes comparable to or larger than the wavelength of the light (Mie, 1908). The Mie solution is a two-lobes function, with a forward and a backward lobe. The ratio between the intensities of the forward and the backward lobe increases with the size of the particles. The Mie solutions are exact but not always analytical, which implies the use of numerical methods to use this model. In addition, the exact solution is restricted to isolated spherical particles, which makes it relevant for atmospheres but not for surfaces. Other representations of the single scattering by particles are thus generally used when analysing data from surfaces, the Henyey-Greenstein function (Henyey and Greenstein, 1941) being the most popular. 
This is a purely empirical and analytical function and its parameters are generally set as free parameters in a model to fit measured data. Different versions exist with one, two, or three parameters. The most widely used is the two-parameters version that allows the definition of a forward and a backward lobe with variable relative intensities, as in the Mie theory, and a single and variable width parameter that applies to both lobes. The description of the scattering of light by the individual particles is completed by the definition of the single scattering albedo (SSA) that quantifies the ratio between the total scattered radiation and the total absorbed radiation by one particle.

The "macroscopic roughness" of the surface, defined as the roughness at all scales larger than a few times the size of the grains, has a strong effect on the reflectance at moderate to high phase angle. The influence of large-scale roughness is derived in an approximated way by Hapke (1984). The rough surface considered is made of facets with a Gaussian slope distribution function. The mean slope angle, theta-bar, is the single parameter used to describe this slopes distribution. It is the mean slope angle over all distances, from a few times the particle sizes up to the resolution of the detector. This can represent a very large range of scales, from less than a millimetre to kilometres in some cases, spanning more than six orders of magnitude. The derivation of the effect of roughness involves other assumptions. The mean slope angle must be small: no vertical scarps or overhangs are considered. The reflections of light from one facet to another are neglected, which is only valid for low surface albedo and/or low roughness. The slope distribution is independent of the azimuth angle. This is generally true but there are some exceptions such as fields of aligned dunes and in general topography generated by dominant winds from a particular direction.

Although it has long been noticed experimentally that the porosity has an effect on the bidirectional reflectance, it is only in 2008 that this parameter was introduced in Hapke's model (Hapke, 2008). Predictions from the updated model show good consistency with experimental data.

One of the most well-known photometric effects is the opposition effect, the increase of the reflectance as the phase angle decreases. It is observed on the surfaces of most solid objects of the Solar System and nearly every particulate sample in the laboratory. A relatively simple explanation to this effect by the Shadow Hiding Opposition Effect (SHOE) was mentioned earlier. Another mechanism susceptible to contribute to the opposition effect is the Coherent Backscattering Opposition Effect (CBOE) that results from constructive interferences between two rays propagating along the same optical paths but in opposite directions. Despite the apparent simplicity of these two explanations to the opposition effect, they do not appear in the approximate solutions to the radiative transfer 
equations used in the Hapke model. Ad-hoc additions to the model designed to account for these effects are thus implemented, which is one of the strongest criticisms found in the literature against the model (Shkuratov et al., 2012). It is acknowledged by Hapke (2013) that "The expression for the coherent backscatter opposition effect (CBOE) is undoubtedly the weakest part of the H-model" and that "the CBOE is poorly understood".

Even if they do not appear in Hapke's formalism, physical explanations and numerical solutions for the coherent backscattering actually exist, such as the one proposed by Shkuratov and Helfenstein (2001), Shkuratov et al. (2002), Muinonen (2004) and Muinonen et al. (2012). Note that the results of Muinonen et al. (2012) contradict two hypotheses often made about the coherent backscatter opposition effect: this effect, as multiple scattering in general, is not restricted to bright surfaces and it can be sensitive on a range of phase angles of $10^{\circ}$ to $20^{\circ}$, much wider than what is generally assumed.

\subsubsection{Summary of relevant Solar System observations}

In this section, we list and comment the observations of water ice associated to contaminants of various nature at the surfaces of different Solar System objects that are the most relevant for the experiments we conduct in the laboratory. The list is not exhaustive and is rather a subjective selection of the observations that have stimulated or will stimulate our experimental work and/or observations that our work could help to better interpret.

\section{Mercury and the Moon}

The floors of some craters close to the poles of Mercury remain permanently shadowed and only receive minute amounts of light scattered from the surrounding crater rims. In these conditions, the soil temperature remains well below $100 \mathrm{~K}$ at the surface and water ice is stable for billions of years (Paige et al., 2013). Water brought to the surface by impacting asteroids and comets can migrate toward the poles and be cold-trapped in permanent shadowed craters. The situation is similar on the Moon (Schorghofer, 2014; Prem et al., 2015). Evidence for polar ice on Mercury was first obtained from radar Earthbased observations in the form of strong and highly unpolarised reflections (Harmon and Slade, 1992; Slade et al., 1992) long before data from the first orbital mission to Mercury, Messenger, confirmed its presence (Neumann et al., 2013; Lawrence et al., 2013). At the centimetric wavelengths used, the reflection of the radio wave is influenced by the properties of the first tens of centimetres to meters below the surface. The Mercury Laser Altimeter (MLA) of NASA's Messenger mission provided precise topographic data of the North polar area that were used to calculate the evolution of the Solar illumination and 
determine the locations where water ice is thermodynamically stable (Paige et al., 2013). The calculated map of near-surface ice stability shows a remarkable consistency with the Earth-based radar observations. In addition, MLA was able to measure the surface reflectance of the radar-bright surfaces at the $1064 \mathrm{~nm}$ laser wavelength. High values of reflectance were observed in the southern portion of crater Prokoviev, one of the largest permanently shadowed areas. It is thus hypothesized that water ice is exposed at the surface in this region, in agreement with the thermal model. Other areas showing strong radar reflectivity but located at lower latitudes appear dark to MLA with a surface reflectivity lower by a factor of 2 to 3 than the average reflectance of Mercury. In these regions, water ice is thermally unstable at the surface but stable below a $10 \mathrm{~cm}$-thick layer of desiccated material providing thermal insulation. It is hypothesized by Neumann et al. (2013) that the surface dark deposits are rich in organic material. Both the water ice and the organics are brought to the surface of Mercury by comets or volatile-rich asteroids. Another instrument of Messenger, its Gamma-Ray and Neutrons Spectrometer (GRNS) also provides evidence for water ice by detecting hydrogen in the first meter below the surface over the north polar region (Lawrence et al., 2013). Due to its very elliptical orbit, only the North pole of Mercury could be studied in detail by Messenger. Analyses of the south polar regions will be performed in 2024 by ESA's BepiColombo Mercury Planetary Orbiter (MPO) spacecraft equipped in particular with the BEpi-Colombo Laser Altimeter (BELA, Thomas et al., 2007).

The case of the Moon seems relatively similar to the one of Mercury at first glance. The presence of water ice at some depth in the soil is attested by both radar (Spudis et al., 2010) and neutron (Miller et al., 2012) instruments, although the radar data might be affected by the roughness of the surface, which makes the identification of ice from this technique only tentative (Thompson et al., 2011; Eke et al., 2014). No strong and systematic brightening of the floor of polar craters is detected however. A noticeable increase of the reflectance of the floor and interior walls of Shackleton crater is observed but is unlikely to be caused by the presence of ice at the surface (Zuber et al., 2012). A maximum amount of 22 wt. \% of $\mathrm{H}_{2} \mathrm{O}$ ice mixed within the regolith is derived from this observation. Recently, the re-analysis of infrared spectroscopic data collected by the M3 instrument of ISRO's Chandrayan spacecraft showed the presence of water ice within permanently shadowed craters at latitudes larger than $75^{\circ}$ in both hemispheres. The estimated amount of water ice mixed intimately with the regolith is of the order of $30 \mathrm{wt}$ \% (Li et al., 2018). The attested presence of water ice excites prospects for future in-situ resource utilization.

\section{Mars}


Water in its liquid and/or solid form was once abundant at the surface of Mars (Carr and Head 2015) but today's surface is mostly dry with the largest visible accumulations of water ice at the poles. AlthoughCO $\mathrm{C}_{2}$ dominates the atmosphere of Mars and can condense to form the large seasonal caps that cover a large fraction of the surface, water has played a major role in the geological history of the red planet and some of today's surface reservoirs of water ice play a significant role in the Martian climate.

In summer, as all the seasonal $\mathrm{CO}_{2}$ ice has sublimated, a large amount of relatively pure water ice can still be observed over the North pole. The visible bright ice constitutes the most surficial of a $>3 \mathrm{~km}$-thick and $>1000 \mathrm{~km}$-wide stack of icy layers. The ice-to-dust ratio of the layers, which controls their brightness and colour as well as their resistance to erosion, is seen to vary cyclically. These variations appear correlated to past changes in the orbital parameters of Mars, suggesting an astronomical forcing of the climate (Beccera et al., 2017). In the southern hemisphere, another permanent cap of similar dimensions is found over the pole but the ice is almost entirely hidden by a thin layer of dust. Water ice only appears at the surface on the steep cliffs of the spiralling troughs (Bibring et al., 2004).

Although $\mathrm{CO}_{2}$ ice condensed from the major atmospheric gas constitutes the bulk of the seasonal caps, $\mathrm{H}_{2} \mathrm{O}$ stills plays a significant role in the evolution of the Northern seasonal cap as it recedes in Spring. Indeed, water ice is seen to progressively cover the layer of $\mathrm{CO}_{2}$ ice in early-mid spring, either because the minor amounts of $\mathrm{H}_{2} \mathrm{O}$ ice that were contained in the $\mathrm{CO}_{2}$ ice accumulate at the surface while $\mathrm{CO}_{2}$ sublimates or because water vapour condenses onto the $\mathrm{CO}_{2}$ ice to form a surficial layer of frost (Appéré et al., 2011). This layer of water ice may prevent the seasonal cap in the northern hemisphere to develop a similar activity as the seasonal cap in the southern hemisphere (Pommerol et al., 2013).

NASA's Mars odyssey spacecraft carries a suite of gamma-ray and neutrons spectrometers that measured the average chemical composition of the first meter below the surface, including its hydrogen content (Feldman et al., 2004). From these data, a global map was produced, showing the hydrogen content converted to a weight percent of equivalent water assuming that all the hydrogen is in the form of $\mathrm{H}_{2} \mathrm{O}$. High values of hydrogen content at latitudes higher than about $60^{\circ}$ in both hemispheres are indicative of the presence of a shallow permafrost, which is consistent with our understanding of the current stability zone of ice in the subsurface (Schorghofer, 2008) and the occurrence of surface features indicative of ground ice (Mangold et al., 2004). High concentrations of hydrogen at equatorial and tropical latitudes are more puzzling and seem to indicate the presence of an unidentified hydrated component at shallow depths. 
The presence of water ice at high latitude, a few centimetres below the surface as indicated by Odyssey's instruments was later confirmed in-situ at one location by NASA's Phoenix lander that found a water ice table at depths between 5 and $18 \mathrm{~cm}$ below the surface in good agreement with Odyssey's estimates. Interestingly, the ice was clearly in excess of the soil porosity, nearly pure at places, confirming as well the very high amounts indicated by Odyssey's instruments.

In addition to ground ice, frost and ground fog as well as higher water ice clouds and snow precipitations were also observed at times of the mission (Smith et al., 2009). Prior to the Phoenix observations, morning frost had been observed in-situ as well by the Viking landers (Jones et al., 1979). Morning fog on Mars has also been observed by various orbiters (for instance Möhlmann et al., 2009). Fog and frost are not only interesting as elements of the diurnal and seasonal water cycle but can also be related to the presence of ice and liquid water or water of hydration in the subsurface.

While Odyssey's instruments and Phoenix observe a shallow ice table, a few centimetres to decimetres below the surface, there are numerous direct and indirect evidences for deeper subsurface ice. Most of the indirect evidences are geomorphologic, consisting in various textures and features produced by the accumulation and sublimation of ice: mantled terrain, viscous flow features, pedestal craters, scalloped terrain, polygons... They are indicative of accumulations of several decametres of ice-rich material under different climatic conditions followed by partial or total sublimation under current climate. Water ice in some of these features could be probed by the SHARAD sounding radar on Mars Reconnaissance Orbiter. A radar reflectivity map produced by the MARSIS sounding radar of Mars Express could also indicate the presence of large amounts of water ice below the plains surroundings the North pole (Mouginot et al., 2012). Recent data from MARSIS also seem to indicate the presence of liquid water below the southern permanent ice cap (Orosei et al,, 2018). Finally, direct optical observations of ice-rich layered deposits have been reported recently been reported by Dundas et al. (2017).

\section{Asteroids}

Telescopic surveys of asteroids have revealed a large diversity in their surface spectro-photometric properties. The most abundant of all asteroids, of the C-type (Tholen, 1984) display very dark and slightly reddish surfaces with specific spectral signatures of aqueous alteration in the VIS-NIR spectral range (Fornasier et al., 2014). In addition to water-of-hydration, a number of asteroids are suspected to have water ice at or below their surfaces, in particular 24-Themis (Rivkin and Emery, 2010; Campins et al., 2010) and 65Cybele (Licandro et al., 2011) although an alternative explanation involving the presence 
of the mineral goethite has been proposed for the case of 24-Themis (Beck et al., 2011). The largest known asteroid, 1-Ceres, is known to emit plumes of water vapour into space (Küppers et al., 2014). Detailed exploration by the Dawn mission revealed a very ice-rich crust and bright spots due to high concentration of salts, likely the hydrated magnesium sulphate hexahydrite associated to ammonia-bearing clays (Nathues et al., 2015; de Sanctis et al., 2015). These bright deposits could be residues of the sublimation of brines erupted from the subsurface.

\section{Comets}

Although the activity of comets is caused by the sublimation of ices, with $\mathrm{H}_{2} \mathrm{O}$ often dominating, the surfaces of the nuclei observed so far from spacecraft or groundbased telescopes display a very low visible albedo, in the range of 2 to 5 percent (Lamy et al. 2004; Barucci et al. 2011). The volatile components are indeed strongly depleted at the surface due to sublimation. The uppermost layer of the nucleus, which interacts with solar radiation, is thus essentially composed of refractory organic and mineral matter. Two mechanisms may be involved in the production and evolution of this surface mantle: persistence of a sublimation lag after the ice has sublimated and/or accumulation of dust lifted up from the surface by gas drag and later redeposited at a distant location (Thomas et al. 2015a,b; Davidsson et al. 2015).

The surfaces of known cometary nuclei are relatively homogeneous but some of them still show significant variability in their surface albedo and spectral properties. In particular, the nucleus of comet 9P/Tempel 1 displays regions about $30 \%$ brighter than surrounding areas at visible wavelengths (Li et al. 2007, 2013). Infrared spectra obtained by the spectrometer of the Deep Impact spacecraft indicate that the bright areas display in addition subtle but clear spectral signatures of $\mathrm{H}_{2} \mathrm{O}$ ice at 1.5 and $2.0 \mu \mathrm{m}$. Inversion of these spectra indicates 3 to $6 \mathrm{wt} . \%$ of $\mathrm{H}_{2} \mathrm{O}$ ice agglomerates at the surface with diameters in the range of 10 to $50 \mu \mathrm{m}$ (Sunshine et al. 2006). Observations of the nucleus of comet 103P/Hartley 2 by the same spacecraft also showed regions of the nucleus with a reflectance about twice the average reflectance of the surface and a bluer visible slope ( $\mathrm{Li}$ et al. 2013). These observations, by comparison with what has been observed on 9P/Tempel 1 , are also indicative of the presence of $\mathrm{H}_{2} \mathrm{O}$ ice at the surface in these regions (Sunshine et al. 2012).

Since the completion of the Rosetta mission, the nucleus of comet $67 \mathrm{P} /$ Churyumov-Gerasimenko is the best-known cometary nucleus and observations of the passage at perihelion of the comet have been particularly insightful for understanding the behaviour of water ice at the surface. Early observations by the OSIRIS visible imaging 
system and the VIRTIS visible and near-infrared hyperspectral imaging system have revealed the occurrence of numerous metre-scale ice-rich patches homogeneously distributed over a typical low albedo homogenous surface (Pommerol et al., 2015, Filacchione et al., 2016). The reflectance spectra measured by VIRTIS show very strong signatures of water ice. Inverse modelling of these spectra indicates millimetre-sized pure water-ice grains. The hypotheses favoured by the authors to explain the occurrence of these ice grains is the growth of grains by vapour diffusion or sintering below the uppermost desiccated layer.

De Sanctis et al. (2015) showed the existence of a water cycle on the nucleus. Ice sublimates from the surface as well as from the close subsurface within the thermal skin depth. At night, the surface cools down, quickly stopping sublimation but the close subsurface remains warmer, inverting the temperature gradient. Ice continues sublimating from the subsurface but part of the emitted vapour condenses at the surface, forming a surficial layer of frost. This frost quickly sublimates when the Sun rises but is visible to VIRTIS along the terminator. Fornasier et al. (2016) have studied the changes in the visible spectro-photometric of the surface of the nucleus after its passage of perihelion. They observe a significant change of colour, the surface becoming bluer overall. The favoured interpretation of this observation is that high activity around perihelion has cleaned the surface of the nucleus from some of the accumulated dust, revealing fresher material below that still contains some amount of water ice. Two large and bright ice-rich patches also appeared and then vanished within ten days as the ice sublimated quickly. Finally, thin layers of water frost are observed following shadows over different regions of the nucleus, illustrating again the water cycle first described by De Sanctis et al. (2015).

\section{Icy moons}

With a few notable exceptions such as Io, Phoebe and Callisto, water ice dominates most of the surfaces of the large icy satellites of the giant planets. Their surfaces look remarkably different however, due either to differences in the properties of the ice itself or the nature and amount of contaminants.

The surface of the satellites of Saturn has been characterized in detail by the remote sensing instruments of the Cassini mission, in particular the VIMS instrument (Brown et al., 2004) that provided new insights into the composition of the surfaces (Scipioni et al., 2013; Scipioni et al., 2014). Enceladus displays the brightest surface in the entire Solar System with a bolometric Bond albedo of 0.85 , corresponding to a surface composed of essentially pure water ice (Pitman et al., 2010). As the surface reflects most of the solar radiation that it receives, its temperature remains very low, of the order of $70 \mathrm{~K}$ (Hanel et 
al., 1982), preventing thermal metamorphism of the ice. Close observations by different instruments on the Cassini spacecraft revealed a high activity at the south pole of the moon in the form of discrete jets of material emitted along a network of warm, large and deep fractures informally referred to as "tiger stripes" (Spencer et al., 2006; Spitale and Porco, 2007; Nimmo et al., 2007). This activity is responsible for the production of small ice particles that compose Saturn's E-ring. Some of these particles re-impact the surface of Enceladus and coat it. Over time, particles of ice are observed to decrease in size because of mechanical weathering by microimpacts and sputtering (Jaumann et al., 2008). The ice is mostly crystalline but amorphous ice is observed over the young regions in between the tiger stripes, suggesting a mechanism to actively produce amorphous ice either by intense radiative bombardment or fast freezing or condensation of liquid water or water vapour, respectively (Newman et al., 2008).

The four large spherical moons Rhea, Dione, Thetys and Mimas show very high visible albedo as well, but also some traces of contaminants responsible for subtle variations in the albedo and colour of the surface (Scipioni et al., 2013; Scipioni et al., 2014). Iapetus, the third largest moon of Saturn presents a spectacular dichotomy in its surfaces properties. Its trailing hemisphere and polar regions are bright, with an albedo of about 0.6 , indicating a surface dominated by ice. The leading hemisphere however is as dark as comets with an albedo lower than 0.05 and a red spectral slope. The origin of this dichotomy can be explained by sublimation of the ice from the dust-contaminated regions that warm up by absorbing most of the solar light and by recondensation of ice on the bright and cold regions of the surfaces. (Denk et al., 2010; Spencer and Denk, 2010). The other small irregular moons show variable surface spectra, indicative of variable amounts of dust mixed with water ice. The irregular moon Phoebe shows particularly interesting surface properties with a generally very dark and reddish surface punctuated by small local exposures of bright water ice, as well as carbon dioxide ice (Hansen et al., 2012). These unusual surface properties as well as the orbital characteristic of Phoebe have led to the hypothesis that it is a captured centaur, a family of minor planets orbiting the Sun in between the orbits of the giant planets (Johnson and Lunine, 2005).

The surfaces of two Galilean satellites, Europa and Ganymede are dominated by water ice, but much less clean and pristine as the one found at the surface of the large spherical moons of Saturn. Europa shows the younger and brighter surface with an albedo of 0.67 but contrasted patterns of albedo and colour across its surface. Interestingly, some of the albedo and colour patterns show a strong correlation with the geomorphology of the surface. This is the case of the linae, which are long and narrow dark and red streaks observed on a large fraction of the surface and correspond to tectonic features that might 
transport warmer ice from the interior to the surface. This leads to the possibility that the dark material observed there is endogenic, possibly produced within Europa's internal ocean. This material contains hydrated compounds, possibly hydrated salts such as $\mathrm{Mg}$ and Na-bearing sulphates or carbonates, interpreted as possible evaporate deposits (McCord et al., 1998a,b). The surface is however strongly contaminated by exogenic material, in particular sulphur emitted from Io's volcanoes. In addition, space weathering in the highly energetically environment of the vicinity of Jupiter is very efficient and sputtering of the surface by electrons and ions is likely to modify the material exposed at the surface. Carlson et al. (1999) have argued that the hydrated compounds were more likely to be hydrated sulfuric acid produced by radiolysis from sulphur polymers than the previously hypothesized sulphate and carbonates salts (McCord et al., 1998a,b). It was also recently shown experimentally that the irradiation of initially white sodium chloride by energetic electrons results in a change of colour of the salt to a brown-yellow that resembles the colour observed in the Linae at the surface of Europa (Hand and Carlson, 2015; Poston et al., 2017).

One of the most noticeable surface features on Ganymede is the presence of bright and extended (down to $40^{\circ}$ latitude) polar caps that were explained by Khurana et al. (2007) as a result of sublimation of the ice induced by ion sputtering and re-deposition as finegrained frost. Bright regions corresponding to heavily tectonized features are also observed (Belton et al., 1996) but the process that produces and maintains the difference of albedo is unclear. The surface of Callisto is highly contaminated by dark matter, sometimes totally masking the signatures of water. The dark contaminant at the surface of Ganymede and Callisto shows the signatures of $\mathrm{Fe}^{3+}$-bearing minerals as well as sulphur dioxide originating from Io's volcanoes but the exact composition remains uncertain (Showman and Malhotra, 1999).

Near-infrared hyperspectral cubes of the surfaces of Europa, Ganymede and Callisto obtained by the Near Infrared Mapping Spectrometer (NIMS) of Galileo were analysed to map the occurrence of amorphous and crystalline water ice from the shape of the 3- $\mu \mathrm{m}$ absorption band. The ice is predominantly amorphous on Europa and crystalline on Callisto whereas both types of ice are observed on the surface of Ganymede. The spatial pattern observed at the surface of Ganymede, the measured surface temperatures and the overall gradient of crystallinity with increasing distance from Jupiter indicate that the occurrence of crystalline versus amorphous ice is controlled by a balance of thermal crystallization and amorphisation induced by radiation (Hansen and McCord, 2004). Recent studies using ground-based telescopes have however challenged this view, 
interpreting the spectra of the surface of Europa as indicative of the presence of crystalline ice rather than amorphous (Ligier et al., 2016).

The moons of Neptune and Uranus are not as well studied as the ones of Jupiter and Saturn, as no dedicated mission has ever orbited the two ice giants. Most of these satellites show much lower albedos than the large moons of Jupiter and Saturn but still present the spectral signatures of water ice in the near-infrared (Brown and Clark, 1984; Brown, 1983). The only colour variability identified on the satellites of Uranus is that craters and impact ejecta appear brighter and less red than the rest of the surface (Buratti and Mosher, 1991). Water ice was detected on the satellite of Neptune Nereid (Brown et al., 1998) but not on Proteus.

\section{Trans Neptunian Objects (TNO)}

Extensive surveys of TNOs have revealed an extreme diversity of albedo, from 0.03 to 1 , and a colour varying between red and almost neutral (Lacerda et al., 2014). Some of the large TNOs with albedo close to 1 and neutral colour are likely to be covered by nearly pure ices. Because of the very cold surface temperatures, around and below $50 \mathrm{~K}$, different ices can be stable including nitrogen, methane, ammonia, carbon monoxide and dioxide in addition to water ice. Crystalline water ice has been identified by infrared spectroscopy on a few of these objects only, including Kuiper Belt Object (50000) Quaoar (Jewitt and Luu, 2004), the TNO 136108 (Merlin et al., 2007), (145453) 2005 RR43 (Pinilla-Alonso et al., 2007) and plutinos (90482) Orcus (Barucci et al., 2008) and 2004 DW (Fornasier et al., 2004). The majority of the TNOs seems to present a thick mantle of complex organics at their surface masking the ice signatures (Brunetto et al., 2006). Brown et al. (2012) provide a review of the observations of water ice in the Kuiper belt.

The flyby of Pluto and Charon by NASA's New Horizons spacecraft in July 2015 provided a wealth of new information on these objects. The geological diversity found at the surface of Pluto is certainly unexpected and the observation of apparently very young surfaces raises important questions on present sources of internal energy (Stern et al., 2015). Although the surface is dominated by nitrogen ice, near-infrared data reveal local exposures of water ice. It was already known before the New Horizons flyby that contrary to the surface of Pluto, the surface of Charon is dominated by water ice. The flyby data reveal that parts of the surface of Charon are relatively young as well, with a dichotomy of age and morphology between the northern and the southern hemisphere and a very large canyon over the boundary. 


\subsection{The IceLab/LOSSy Project - Motivations, history and objectives}

Our intentions to measure the spectro-photometric properties of well-characterized ice/dust mixtures translated into the construction on a new laboratory at the University of Bern, entirely focused on this objective. The development of the Ice Laboratory at the University of Bern started in 2010 with the design and construction of a gonio-radiometer able to work at negative temperatures based on a previous development, the Physikalisches Institut Radiometric Experiment (Gunderson et al., 2006; Gunderson et al., 2007; Gunderson and Thomas, 2008). In addition to being installed in a large laboratory freezer, the PHIRE design was improved in many ways to enhance its performances and facilitate its use (Pommerol et al., 2011). Then, the scientific priorities of the laboratory were related to the Martian mission Mars Reconnaissance Orbiter, the cometary mission Rosetta and the Mercury mission BepiColombo because of the involvement of the team in the visible imaging and laser altimetry instruments on these missions.

In its initial form, the PHIRE-2 instrument was able to measure the bidirectional reflectance of a sample under a large variety of incidence, emission and azimuth angles. A strong limitation however, was the impossibility to measure the reflectance at phase angle below $4^{\circ}$. The most important late improvement to the PHIRE-2 setup was therefore the development of a new detector head equipped with a beamsplitter, which allows us measuring the reflectance at phase angle $<1^{\circ}$ (Jost et al., 2016).

Although the development of the new laboratory started with the construction of its main measurement facility, it was recognized from the beginning that the production and characterization of the samples would be the most challenging part of the project but also, by many aspects, the most interesting scientifically. Of particular interest was the evolution of samples exposed to conditions of low pressure and temperature. We have thus built next to the PHIRE-2 instrument, the SCITEAS (Simulation Chamber for Imaging the Temporal Evolution of Analogue Samples) facility in which the samples could be exposed to vacuum at low pressure to simulate sublimation under space conditions (Pommerol et al., 2015). The PHIRE-2 gonio-radiometer and the simulation chamber were designed to permit the quick exchange of the samples between both facilities by using a standard cylindrical sample holder ( $16 \mathrm{~cm}$ diameter, $25 \mathrm{~cm}$ height), which is used for all facilities in the laboratory. The upper lid of the chamber was equipped with a large quartz window and its bottom flange with a variety of electrical, mechanical and optical feedthroughs. An original visible and near-infrared hyperspectral imaging system was built to monitor the surface of the sample through the window. The hyperspectral imaging system can be used to measure reflectance spectra of samples (see figures 7 and 8 in El-Maarry et al., 2015 for 
instance) or to simulate the signal that would be measured by colour imagers by convolving the measured hyperspectral cubes by the spectral responses of the instruments.

The first samples analysed in the IceLab were procured through collaborations with other groups in Europe. Among the first samples measured on PHIRE-2 were some meteorite powders that provide interesting data for comparison with asteroid surfaces (Beck et al., 2012). Following series of tests measurements with various forms of water ice (fresh snow, crushed ice) that are easy to procure but of low relevance for planetary science, we have acquired reflectance data on samples of micrometre-sized water ice produced using a setup developed at the University of Braunschweig (Gundlach et al., 2011b). The water ice produced using this setup is interesting for two main reasons. First, it is representative of the water ice present in different astrophysical environments, in particular the nucleus of comets or the surface of active icy satellites. Second, the production procedure is repeatable and the setup can be transported to be used in different facilities. This setup was used to produce the ice used for measurements reported by Jost et al. (2013).

Two events have strongly affected the evolution of the project in terms of definition of its scientific objectives and allocated resources since it started: the foundation of the Center for Space and Habitability at the University of Bern in 2013 and the National Center for Competence in Research (NCCR) PlanetS in 2014. In the framework of the CSH and through collaboration with a team of biologists in Bern, we have started studying the reflectance properties of biotic and abiotic organic matter and define possible biosignatures in the reflected light from planetary and exoplanetary surfaces. The first results were recently published by Poch et al. (2017). In the framework of the NCCR PlanetS, we are investigating the possibility to apply our experiments and data to the field of the origin of planetary systems. We have also developed new capabilities to measure polarization in the reflected light from icy samples in response to the new thematic opened up by the two centres.

Indeed, until recently one important aspect of photometry had not been studied in the Planetary Ice Laboratory: the polarization of the light. This was without doubt a gap in our measurement capabilities that we have decided to fill by developing the POLarimeter for ICE Samples (POLICES) setup (Poch et al., 2018). It consists of an accurate Stockes polarimeter completed by a steerable light source setup and a cryogenic sample holder. Beyond our general scientific objectives, to which the POLICES setup will contribute greatly, the setup was designed to fulfil two specific and new objectives. The first one is the measurement of polarization in the reflected light from icy samples containing biotic and abiotic organic matter. The goal is to assess the potential of visible circular polarization as a possible biosignature at the surface of icy moons and planets in the Solar System 
(Europe, Ganymede, Enceladus) or in extrasolar planetary systems in the continuity of the work initiated by Poch et al. (2017). The second objective is the measurement of the polarized phase curves of various ice/dust mixtures. These laboratory data should be useful for interpreting recent observations of planets and protoplanetary or debris disks in visible polarized light such as the ones recently acquired by SPHERE (Engler et al., 2017) and new observations of the surfaces of Solar System objects and in particular the icy moons of Jupiter by the same instrument.

In addition to our initial focus on the VIS-NIR spectral range, we have also started investigating the electrical properties of the icy samples at microwave wavelengths. This decision was motivated by the strong complementarity between optical and radar/sub-mm techniques in a variety of context. We proceeded through collaboration with specialists at the University of Aix-Marseille who brought to our laboratory in Bern their setup to perform these measurements (Brouet el al., 2016a). The experimental setup consists of a conical coaxial cell connected to a vector network analyzer (Sabouroux et al., 2011; Georget et al., 2014). As described in Section 4.5, the characterization of the electrical properties of analogue materials in the laboratory under controlled conditions is primordial to assess the technical performances of radars (as Ground Penetrating Radars or bistatic radars), as well as to determine the internal physical properties and composition of a planetary object from a radio instrument onboard a spacecraft or onboard a rover, as they control the radiative transfer of the radio waves in planetary media.

We have followed a similar approach to complement our series of optical measurements with complementary data using various techniques through collaborations with teams of experts in the various domains. Most of the time and contrary to the previous example, the measurements facilities cannot be moved and we rather transport the setups we have developed to produce the icy samples (see Section 3). That way, we have recently transported our sample production facilities to two other laboratories in Bern to perform sputtering experiments (Galli et al., 2016) and measurements of the dielectric permittivity at $\mathrm{mm} / \mathrm{sub}-\mathrm{mm}$ wavelengths $(150-210 \mathrm{GHz})$, complementing those previously performed at longer wavelength in our laboratory. Two of the ice production setups were also transported to the Centre Suisse d'Electronique et de Microtechnique (CSEM) in Neuchâtel to acquire cryo- Scanning Electron Microscope (SEM) images of the samples at sub- $\mu \mathrm{m}$ resolutions that allowed us to characterize their grain size distribution quantitatively and document the texture of the surface of the grains as well as their evolution with time (Jost et al., 2016). Recently, three ice production setups were transported to the Institut de Planétologie et d'Astrophysique de Grenoble (IPAG) to acquire reflectance spectra in the mid-infrared, at longer wavelength than what is possible in our laboratory in Bern. 


\section{Sample production and characterization}

\subsection{Sample production}

\subsubsection{The SPIPA setups}

Among our main objectives with this project was the development of a suite of reproducible and well-characterized samples made of ice and dust mixed in different ways. Analogues for the most refractory components of the dust and readily available and used. For instance, the lunar and martian regolith analogues initially developed and distributed by NASA's Johnson Space Center, JSC1 (McKay et al., 1993) and JSC Mars-1 (Allen et a1., 1997), respectively. More recently, analogues for asteroid regolith have been proposed (Britt et al., 2017) and are now distributed commercially (http://deepspaceindustries.com/simulants/).

More problematic are the provision and handling of the volatile and semi-volatile components. Fine-grained ice samples cannot be easily stored and transported at the very low temperature required for them to remain pristine, as they are prone to metamorphism. Semi-volatile organic components are also often unstable at room temperature and many of them are poisonous. The solution we have adopted to produce the water ice component of the samples is to build portable machines that can produce the water ice samples in a reproducible way. Rather than transporting the ice samples, the machines can be moved from one laboratory to another and new samples prepared in-situ as needed. We refer to this machines as the Setups for Production of Icy Planetary Analogues (SPIPA). These setups can be used in two ways, either to produce pure water ice particles that can later be mixed with dust or to produce particles consisting of a suspension of dust grains in a matrix of ice. We refer to these two sorts of mixtures as inter- and intra-mixtures, respectively. We first describe the setups here and how they are used to produce intra-mixtures before detailing the procedure to prepare inter-mixtures of ice and dust. Electronic Supplementary Material 1 also lists the hardware needed to re-build the SPIPA setups.

\section{SPIPA-A}

The main concept of this setup is to nebulize liquid water and freeze the droplets in a cold environment (Figure 2). Water is inserted in a small plastic cup and nebulized from an ultrasonic generator below. It is possible to insert contaminants in the water as long as they are soluble at room temperature (e.g. salts). According to the manufacturer, the produced droplets have a diameter in the range of $0.47-6 \mu \mathrm{m}$. The nebula leaves the device via an exhaust and is conducted through a large plastic tube ( $4 \mathrm{~cm}$ diameter). 
The tube is guided into a slightly modified chest freezer. As the freezer can only cool down to $-40^{\circ} \mathrm{C}$, we place a small dewar with a massive copper plate on top inside the freezer. It is (re-)filled with liquid nitrogen about half an hour in advance and sets the air temperature to about $-80^{\circ} \mathrm{C}$. This low temperature reduces thermal metamorphism of the ice particles. A steel bowl is placed on the copper plate, in which the sample will accumulate. To prevent condensed water inside the tube to drip directly into the accumulation bowl, the tube is guided in two U-turns through the freezer. At the lowest point, we have cut a small opening in the tube to release liquid water.

As the flow of the droplets is driven solely by gravity, it is necessary to leave in the freezer cover a slit open during the production to avoid blocking the airstream inside the tube. The slopes of the walls of the collection bowl play a similar role. The walls should not be vertical to ensure a certain flow. With an optimized layout, the SPIPA-A setup is able to produce about $50 \mathrm{~g}$ of sample material per hour. The particle sizes of fresh ice particles have a mean diameter of $4.54 \pm 2.54 \mu \mathrm{m}$ (Jost et al. 2016). The size distribution is log-normal shaped.

Liquids with a viscosity close to the one of pure water $(1 \mathrm{Cp}$; for example, water solutions with salts, soluble molecules etc. in low concentrations) could be nebulized using the SPIPA-A setup to produce particles of about $4.5 \pm 2.5 \mu \mathrm{m}$. For security reason, because the droplets smaller than $5 \mu \mathrm{m}$ produced with this setup can be readily inhaled and deposited in the lungs, the nebulization of potentially toxic liquid mixtures using SPIPA-A should be made under an extractor hood and/or using special respiratory masks. Galli et al. (2016) produced ice particles from deionized water containing $1 \mathrm{wt} . \%$ of $\mathrm{NaCl}$ using this method. To nebulize liquids with higher content of contaminants, and consequently higher viscosity, we used the SPIPA-B and SPIPA-C setups.

\section{SPIPA-B}

The SPIPA-B setup was originally designed for two purposes: first to provide spherical ice particles with larger diameters than the $\mu \mathrm{m}$-sized particles produced by SPIPA-A and second to permit the production of « intra-mixtures » from suspensions of solid particles in water. To facilitate the suspension or dissolution of the contaminants into the water, the liquid is mixed for several minutes using an ultrasonic mixer equipped with a sonotrode (Figure 3). This sonotrode, plunged into the liquid, transmits ultrasonic vibrations causing acoustic cavitation resulting in a vigorous mixing. If the contaminants are partially or totally insoluble in water, ultrasonic mixing will help to obtain a 
homogeneous suspension, via the break up and dispersion of dust aggregates in the liquid water. For SPIPA-B, we used a Hielscher $200 \mathrm{Ht}$ ultrasonic unit equipped with a 7-mm diameter sonotrode to mix tholins, which are made of water-soluble and insoluble organic molecules, with liquid water (Poch et al., 2016a). The parameters controlling the ultrasonication (intensity, time steps) can be automatically adjusted and recorded, allowing the reproducibility of this mixing phase. For instance, to suspend tholins particles in water, the intensity was fixed at the maximum amplitude of the $7 \mathrm{~mm}$ sonotrode $(100 \%)$ and the ultrasounds were delivered by steps of 0.5 seconds during one minute. Ultrasonication delivers a large amount of energy, resulting in heating of the liquid and possibly chemical reactions to occur. One thus has to take care not to alter the dust component chemically and structurally. During the mixing, a temperature sensor connected to the ultrasonic unit can be plunged into the liquid to monitor the temperature and maintain it below $30^{\circ} \mathrm{C}$. A fraction of the liquid can be collected and dried to perform comparative analyses with nonultrasoniccated dust to check a-posteriori for possible alteration(s) of the contaminant by the ultrasonication. For example, we checked via Scanning Electron Microscopy (SEM) that the structure of the tholins material was not affected after ultrasonication in water described above.

Once prepared, the liquid-dust slurry must be continuously mixed to avoid sedimentation and keep the mixture homogeneous, until it is nebulized. For this purpose, a magnetic stirrer can be used with the vial containing the mixture hermetically sealed using Parafilm to avoid evaporation or contamination.

Liquids with slightly higher viscosity than pure water and containing up to $10 \mathrm{wt} . \%$ of insoluble dust can be nebulized using the SPIPA-B setup. A plastic pipe is plunged into the liquid mixture, maintained under stirring. This pipe is connected to a peristaltic pump that slowly moves the liquid to a special hollowed sonotrode for ultrasonic spraying and nebulization. We used the sonotrode S26d18S connected to the Hielscher 200Ht ultrasonic unit with the amplitude of the ultrasounds at $100 \%$ and the time step of 0.5 second. When the liquid injected in this sonotrode reaches the nozzle, it is dispersed in the form of droplets by the ultrasonic vibrations. The ultrasonic generator and its sonotrode are placed in a freezer, about $10 \mathrm{~cm}$ above an aluminium bowl $(15 \mathrm{~cm}$ diameter) filled with liquid nitrogen so that the water droplets produced by the sonotrode fall into it and are instantly frozen. Note that the nozzle should be positioned slightly off the container to avoid the fall and freezing of large droplets sometimes released by the sonotrode when the liquid flow increases. During the production, care should be taken to maintain a high level of liquid nitrogen in the container to avoid the formation of a crust made by the accumulation of ice particles on the rims. The pumping and nebulization process can last for several minutes 
depending on the volume of the liquid mixture. When nebulizing liquid mixtures containing 1 wt.\% of insoluble dust or more, clogging of the pipe and/or sonotrode may happen. To avoid clogging, it is recommended to heat up the liquid mixture (up to $30^{\circ} \mathrm{C}$, using the heater of the magnetic stirrer for example) and to remove the ultrasonic generator and sonotrode from the freezer when not used. At the end of the production, the ice-dust particles can be used after total evaporation of the liquid nitrogen in the container. This technique allows the production of ice-dust "intra-mixture" spherical particles of $67 \pm 31$ $\mu \mathrm{m}$ in diameter.

\section{SPIPA-C}

Both the SPIPA-A and SPIPA-B ice production setups are built around ultrasonic devices to produce water droplets that are then frozen. We have recently started experimenting with a different technique to diversify the characteristics of the samples produced and enable the productions of "intra-mixtures" (grains of dust included within the grains of ice) with higher dust to ice ratios than what was possible with SPIPA-A and SPIPA-B. To nebulize liquid slurries containing more than $10 \mathrm{wt} \%$ of contaminants or dust, or to produce smaller ice-dust particles from liquids having up to $10 \mathrm{wt} . \%$ dust or more, an air compressing nebulizer should be used. SPIPA-C uses compressed air and a special spraying nozzle to produced samples with variable size at a very high production rate (Figure 4). The spraying setup is connected to a peristaltic pump that provides the liquid. Changing the air pressure and the liquid outflow rate from the pump allows us to produce samples with variable characteristics. We have successfully tested the production of icy samples with up to one third of dust by mass.

Similar to the SPIPA-B setup, a plastic pipe (Tygon R-3603) is plunged into the liquid mixture, maintained under stirring, and connected to a peristaltic pump (PLP 2200, 0.07 to $2200 \mathrm{~mL} / \mathrm{min}$, equipped with the pump head PPH 103) moving the liquid to the nebulizer. SPIPA-C uses a Flow Blurring Metal (FBM-2mm) nebulizer from Ingeniatrics (Ingeniatrics, SL, Seville, Spain) made of stainless steel, which can produce droplets of about 2 to $100 \mu \mathrm{m}$ from liquids of viscosity up to $145000 \mathrm{cP}$. The nebulizer is connected to a dedicated air compressor system consisting of an oil-free compressor (DK50 10Z, from EKOM with a $10 \mathrm{~L}$ tank and a maximum pressure of 8 bar) equipped with a water filter, a $3 \mu \mathrm{m}$ dust pre-filter, a sub-micrometric dust filter and a precision manometer to produce a continuous flow of clean air at a controlled pressure. The nebulizer, clamped on a vertical stand, is positioned on top of an aluminium bowl filled with liquid nitrogen, inside an insulated container (about $23 \mathrm{~cm}$ diameter). The distance from the nebulizer to the surface 
of the liquid nitrogen is about $20 \mathrm{~cm}$. To get a better focusing of the jet and limit the projections of liquid nitrogen and water droplets, a piece of cropped plastic bottle can be connected to the output of the nebulizer and positioned so that its end lies $1 \mathrm{~cm}$ below the surface of liquid nitrogen. During the production, liquid nitrogen should be refilled inside the bowl to keep its level constant. The gas flow is turned ON, then the liquid flow, for maximum 1 minute. After 1 minute, the piece of plastic has to be cleaned from the deposition of ice particles and chunks that prevent a permanent production. After cleaning, the production can start again. Once the desired quantity of ice has been produced, the liquid nitrogen containing the particles is poured into a $800 \mu \mathrm{m}$ metallic sieve, pre-cooled with liquid nitrogen in a second aluminium bowl, to remove the big chunks of ice. Once sieved, the ice particles can be transferred in a smaller bowl, possibly on a warm surface, to speed up the evaporation of the liquid nitrogen.

\section{General remarks concerning the preparation of SPIPA-A, B or C intra-mixtures}

Note that the size of the ice particles produced by these methods depends on the controlled parameters of each setup (liquid flow, ultrasound amplitude, time steps, air pressure) but also on the viscosity of the nebulized liquid. Using the same set of parameters on a sample more viscous than another may not produce identical particle size distributions. Here, the particles sizes are given for liquid mixture having a viscosity close to $1 \mathrm{Cp}$ (viscosity of pure water).

Finally, when producing intra-mixtures of ice-dust particles using insoluble contaminants, the size of these contaminants must obviously be smaller than the average size of the ice particles produced by the SPIPA-A, B or C setup respectively, to make sure the dust particles are correctly included inside the ice particles.

\subsubsection{Production of INTER-MIXTURES}

Inter-mixtures -also intimate mixtures- are homogeneous mixtures of ice and dust at the scale of the individual grains. The production of inter-mixtures is often challenging, since the addition of dust into ice is likely to increase its temperature, and hence accelerate its evolution. This is particularly the case when working with small particles of ice, like the SPIPA A ones, and/or when using dusts with low albedo, since they absorb more incident radiation. A fast working methodology is hence essential for a successful production of icy inter-mixtures. Another key requirement is the homogeneity of the mixtures; the entire 
surface of the sample has to present the same ice-to-dust ratio. Homogeneity of intermixtures will mainly depend upon the grain size and the concentration ratio of the endmembers. End-members with similar size distributions will be easily mixed, and vice versa. At the same time, a 1:1 inter-mixture will be easier to homogenise than a 3:1 one. Last but not least, the reproducibility of the mixtures is imperative to carry out comparisons between experiments. The protocols that we present guarantee the homogeneity and reproducibility of inter-mixtures, as well as the preservation of the low temperature of the samples. Examples of different studies involving intimate mixtures are Yoldi et al., 2015, Poch et al., 2016a,b and Jost et al., 2017a,b (See Section 4).

Prior to the production of the ice, we weight and put, for each sample, the needed amount of dust into aluminium bottles. We then put the bottles into a freezer $(230 \mathrm{~K})$, for at least one hour per decigram of dust. Aluminium is a good thermal conductor even at low temperatures, which guarantees the cooling of the samples, and it is resistant enough to be plunged directly into liquid nitrogen. The sample holders must be pre-cooled as well and we thus generally keep them at $230 \mathrm{~K}$ for several hours in a freezer. Nevertheless, this process can be accelerated by submerging the sample holders into liquid nitrogen. The production of ice is fully automated and lasts about 30 minutes for an average ice production of 20-50 $\mathrm{g}$ of ice (depending on its density). We use that time to prepare the working surface, which is typically composed of a weighing scale, a Vortex-Genie2 mixer and two cryogenic boxes with liquid nitrogen. The aluminium bottles are introduced into one of the cryo-boxes, along with a spoon and a spatula. Once the ice is ready, we take its container out of the freezer and introduce it in the remaining cryogenic box. Liquid nitrogen keeps the ice at low temperature, while the outgassing of $\mathrm{N}_{2}$ keeps the atmosphere dry. One by one, the bottles are put on the weighing scale, filled with the corresponding mass of ice and put back into the box with liquid nitrogen. Next, we proceed with the mixing by agitating the bottle on the mixer platform at full speed (Figure 5). This process is optimized by tilting the bottle $45^{\circ}$ from the platform plane. We introduce the cold spatula in the bottle, so that the particles collide against it and form less agglomerates. We use the vortex during $10 \mathrm{~s}$ and plunge the bottle into $\mathrm{LN}_{2}$ for another $10 \mathrm{~s}$ to avoid the warming up of the sample. This sequence is repeated three times. As mentioned before, if the particles of ice and dust present different size distributions, the mixing of high and/or low ice-to-dust ratios becomes challenging. In those cases, the protocol is slightly modified so that the components are mixed in steps close to 1:1 mass ratio. For instance, when preparing a mixture of $9 \mathrm{~g}$ of SPIPA $\mathrm{C}$ with $1 \mathrm{~g}$ of a fine-grained dust, we would first mix on the vortex $1 \mathrm{~g}$ of ice and $1 \mathrm{~g}$ of dust, then we would add and mix $2 \mathrm{~g}$ of ice to our $2 \mathrm{~g}$ mixture, and finally we would add the 6 remaining grams of ice to the $4 \mathrm{~g}$ of mixture. Once the mixing process is over, we move the cryo-box with the aluminium samples back to the freezer and 
start the filling of the sample holders. Studies such as the one carried by Pommerol et al., $2013 \mathrm{~b}$ have shown the influence of sample preparation in reflectance measurements, and therefore the process of filling the sample holder will fully depend on the aim of the study. A standard procedure would be pouring the content of the aluminium bottle into the sample holder and carefully removing the excess of sample with a spatula. It is up to the operator to decide whether further manipulations must be performed, such as compaction or sprinkling of the sample.

\subsubsection{Frost on dusty surfaces}

The appearance of water frost can be either the result of direct condensation of atmospheric water onto a surface or the deposition onto that surface of icy grains that have condensed in the adjacent atmosphere. We have designed and constructed a sample holder that allows for cold-trapping of atmospheric water onto a dusty surface. Even though the sample holder can be used in both PHIRE-2 and SCITEAS, the possibility of purging the SCITEAS chamber and hence to stop frost deposition during measurements makes of SCITEAS the facility of choice for our studies of frost. Figure 6-1, 6-2 and 6-3 show schematics of our sample holder, which is composed of two pieces. The first piece (Figure 6-1) is a cylindrical recipient in which we pour $\mathrm{LN}_{2}$. The second piece (Figure 6-2) is the cover of the cylinder, pierced with a copper piece. The two pieces are assembled as shown in Figure 6-3. Below the cover, the copper piece consists of disks connected through their centre, which maximises the copper-surface in contact with the $\mathrm{LN}_{2}$. The exposed part of copper is a cube of $80 \times 60 \times 20$ millimetres that, because of large heat conductivity of copper, stays below $220 \mathrm{~K}$ for one hour with no need of other cooling systems. As can be seen in Figure 6-4, the rest of the sample holder is covered with an isolating black foam, so that water condensates preferentially on the sample in contact with the cold surface.

Several hours before the beginning of the measurement, we cool down both parts of the sample holder by plunging them into liquid nitrogen. Once the sample holder has reached $\sim 100 \mathrm{~K}$ (close to the boiling point of liquid nitrogen at 1 bar pressure, $77 \mathrm{~K}$ ), we spread the dust over the copper surface in thin layers, and we fill the cylinder with $\mathrm{LN}_{2}$. Usually, we prepare our samples in two-millimetre-thick layers to avoid large thermal gradients within the dust. We then carefully put the two pieces together and place the sample holder in SCITEAS. The chamber is left open for frost deposition, and closed to start the hyperspectral measurements. The outgassing of the $\mathrm{LN}_{2}$ inside the sample holder keeps the atmosphere relatively dry. We can also further purge the atmosphere of the 
chamber by creating a flow of gaseous nitrogen. This way, the deposition of frost is interrupted during the measurement.

Figure 7 shows, on the left, a microscope image of the surface shown in Figure 64. In this case, water frost has grown enough to create needles. Figure 7, right shows an optical coherence tomography (OCT) picture (see Section 3.2.3) of the same sample, where the structure of the frost needles can be appreciated. The quantification of the mass of coldtrapped-water into the samples is challenging, and we do not have, for the moment, the means to do it. Nevertheless, the frost growth on a surface occurs in various steps (Wu et al., 2007), i.e. formation of condensate droplets, droplet growth, freezing of the droplets, formation of the crystals on the frozen droplets and growth of frost crystals, as seen in Figure 6. The identification of the deposition step allows us to assess the thickness of the frost layer on the sample.

\subsubsection{Frozen soils}

To produce frozen soils, the desired amount of dust is weighted and put into sample holders. Gently, we add deionized water by spraying an aerosol of very fine droplets over the surface of the sample. Before and after each addition of water, we measure the mass of the sample to determine its bulk water content. We generally repeat the process until the sample is saturated by water, which is indicated by the appearance of menisci of water around the surficial grains of the samples. Saturating the sample with water is the only way to ensure that it is homogeneously wet. Interrupting the process of wetting before saturation would likely result in vertical gradients of water inside the sample. The wet sample is then left at a bottom of a laboratory freezer at a constant temperature of $243 \mathrm{~K}$ overnight or for longer durations. The result of this process is illustrated on Figure 8a. Invariably, samples produced that way show a matrix of water ice between all the mineral grains in the bulk of the sample but the uppermost grains remain dry and exposed at the surface. An additional step is thus required when a sample fully covered by ice at the surface is desired (such as the samples shown in Figure $8 \mathrm{~b}$ or $8 \mathrm{c}$ ). In this case, we spray fine droplets of liquid water over the surface of the cold sample at the bottom of the freezer. The droplets freeze instantly at the surface of the sample, producing a thin conformal layer of ice at the surface of all grains (Figure 8b). Spraying more water results in thicker films of water until the topography of the underlying grains is lost (Figure 8c). 


\subsection{Quantitative characterization of the produced samples}

\subsubsection{Standard characterization tools}

The bulk density of the samples can be measured by weighting the known volumes of samples contained in sample holder of known dimensions. Additionally, we have purchased a gas pycnometer to measure the porosity of the samples. If the particles are large enough (larger than a few $\mu \mathrm{m}$ ), the samples can be imaged with an optical microscope (Figure 9).

\subsubsection{Cryo-SEM}

The technique of choice to characterize the samples at the scale of the individual grains is Scanning Electron Microscopy (SEM). Some of these instruments can be equipped with a cooled sample holding stage and a special sample introduction system that permit the measurements of ice-bearing samples. This technique was used by Gundlach et al. (2011b) to derive the particle size distribution of their samples and document the aspect of their surface, in particular the presence of water frost at the surface of the grains. CryoSEM images have been acquired for many of the ice and ice/dust mixtures samples described in Section 3.1 at the Centre Suisse d'Electronique et de Microtechnique (CSEM) using a Philips XL30 ESEM-FEG SEM equiped with a Gatan Alto 2500 cryo-transfer stage. The sample was sputter-coated with some nanometers of platinum in the transfer stage to improve its conductivity.

Using these data, Jost et al. (2016) could confirm a hypothesis made when first measuring the photometric properties of the samples prepared by Gundlach et al. (2011b) that attributed to sintering the observed evolution of the phase curves of the samples. Figure 10 shows examples of two intimate mixtures of SPIPA-A water ice particles with particles of the JSC1-AF lunar dust simulant. Both images show how the irregular particles of basaltic dust and the spherical and partially sintered particles of ice come into contact and are well mixed at the particles scale.

\subsubsection{OCTOPUS (Optical Coherence TOmography of Planetary Ultracold Samples)}

To complement the information at the grain scale provided by the SEM, we wanted an instrument able to characterize quantitatively the entire surface of $\mathrm{cm}$-sized samples with a resolution sufficient to identify the individual grains or agglomerates of grains. After having considered different techniques, we have finally acquired an Optical Coherence Tomography (OCT) microscope (Thorlabs Ganymede). OCT is a relatively recent 
technique that provides information on both the surface of the sample and its subsurface by interfering a light beam backscattered from the sample with a light beam traveling through a reference arm. The instrument provides a three-dimensional view of the sample with a lateral resolution of $8 \mu \mathrm{m}$ and a vertical resolution in air of $6 \mu \mathrm{m}$. The lateral dimensions of the image are up to $1 \mathrm{~cm}$. Because this technique uses the baskscattered light to image the subsurface of the sample, the maximum thickness that can be probed depends strongly on the optical properties of the materials. For very transparent materials such as pure water ice, the thickness of the layer probed can reach about 100 particles diameters (Hapke, 1993).

We have installed the head of the OCT instrument on a mobile arm to measure samples in-situ in SCITEAS through the window. Unfortunately, the dark dusty ice sublimates as the beam hits the surface. The measurement is thus destructive for the sample in that case. In addition to the example of the frost shown in Figure 7, Figure 11 shows an example of dataset collected with the OCTOPUS setup on a sublimation residue (see Section 4.1 and Figure 12) made of SWy-2 smectite. The three-dimensional rendering shown in Figure 11 illustrates how the OCT data provide information about the structure of the surface by showing the presence of circular agglomerates with diameters between 50 and $100 \mu \mathrm{m}$.

\section{Results}

Table 5 presents a summary of the measurements already performed and published with various types of ice/dust associations. Most of these results are further briefly described in the following five subsections.

\subsection{Spectrophotometry of ice/dust mixtures and implications for comets.}

Motivated by the context of the Rosetta mission, we performed a series of cometary simulations using the SCITEAS setup, to improve our knowledge of the spectrophotometric properties of sublimating surfaces in the (Vis-NIR) wavelength range. Pommerol et al. (2015) described the first cometary experiment performed: a heterogeneous inter-mixture made of fines and aggregates of SPIPA-A water ice mixed in liquid nitrogen with carbon black (1 wt.\%) and JSC1-AF lunar regolith simulant (volcanic ash) (5 wt.\%) was left to sublimate for 37 hours in vacuum and at low temperature. The sample rapidly lost its surface topography, became smoother at large scale, but rougher at the scale of the dust and ice aggregates. Simultaneously, the visible reflectance decreased while the spectral slope increased because of the accumulation of dark dust over the ice. 
Later, the growth of circular depressions was observed, with bright ice exposed at their periphery. It was also observed that the desiccated crust slowed down the sublimation of the ice located below. As this crust formed, the depths of the water absorption bands decreased, in strong correlation with the increase of the spectral red slope.

This first experiment was followed by a systematic study of the influence of the nature of the dust constituents (mineral or organic) and the way they are initially mixed with the ice (as inter- or intra-mixtures) on the properties of the dust residue produced after sublimation (Poch et al., 2016a,b). This study was in particular motivated by the earlier works of Dobrovolsky and Kajmakov (1977), Saunders et al. (1986) and Storrs et al. (1988) who observed the formation of so-called "filamentary sublimate residues" of very high porosity after the sublimation of ices containing phyllosilicates and/or organic matter. We prepared and sublimated intra- and inter-particles mixtures (see Section 3.1) of SPIPA-B water ice with grains of pure olivine (forsterite, $\leq 80 \mu \mathrm{m}$ ), phyllosilicate (montmorillonite, $\leq 80 \mu \mathrm{m}$ ) and complex organic matter (tholins, $\sim 0.3 \mu \mathrm{m}$ ). The tholins used for this study and the following ones were produced in a plasma of $95 \% \mathrm{~N}_{2}$ and $5 \% \mathrm{CH}_{4}$ within the PAMPRE setup (Szopa et al., 2006; Carrasco et al., 2009). They are analogues of a complex extraterrestrial organic matter, exhibiting a strong spectral slope from 0.2 to $1.0 \mu \mathrm{m}$. All the mixtures we studied produced sublimation residues made of filaments, formed by the aggregation of the non-volatiles dragged by the gas (Figure $12 \mathrm{a}, \mathrm{b}, \mathrm{d}$ ) or concentrated between water crystals (Figure $12 \mathrm{c}, \mathrm{d}, \mathrm{e}, \mathrm{f}$ ). Sudden ejections of fragments of sublimation residues, exposing ice to the surface, were also often observed (Figure 13).

This study demonstrated that the texture (micro-structure, cohesiveness), the activity (in terms of frequency and size of ejected fragments), and the spectro-photometric properties of the sublimation residues (albedo, band depths, spectral slopes) are all very dependent upon a multitude of parameters such as the chemistry of the non-volatiles, their grain sizes, the way they are mixed with the ice and the dust/ice mass ratio of the initial mixture. For example, the structure of residues made of pure olivine grains was very fragile whereas phyllosilicate and organic matter produced residues having higher internal cohesiveness because of their stronger inter-particulate forces. The tholins were found to play a major role in the properties of the sublimation residues, acting as a glue holding silicate grains together and dominating the reflectance by masking the silicates even at lower concentration. In the case of the intra-mixtures, large chunks of a cohesive skin of dust are more often ejected than in the case of inter-mixtures, for which much smaller ejected fragments are observed (see Figure 13). The mantle of refractory dust formed by this process reaches extreme values of porosity, in excess of $90 \%$ for intra-mixtures, which have strong effects on the spectro-photometric properties. These "foam-like residues" exhibit reduced band depths and sometimes nearly featureless negative (blue) spectral 
slopes from 1 to $2 \mu \mathrm{m}$, as observed for a sublimation residue made of phyllosilicates (Figure 14).

On the nucleus of comet 67P/Churyumov-Gerasimenko, metre-sized bright spots observed at the surface by the OSIRIS camera were found to have similar contrasts of brightness and colour (spectral ratios) versus the surrounding surface than ice-exposed versus ice-covered areas on cometary analogues sublimated in the laboratory, confirming the ice-rich content of bright patches (Pommerol et al., 2015b). Further comparison between laboratory spectra and OSIRIS spectra, performed by Oklay et al. (2016), showed that some spectral variability observed at the surface of 67P can be explained by the progressive sublimation of ice-dust mixtures forming a darker layer on the cometary surface.

Jost et al. $(2017 \mathrm{a}, \mathrm{b})$ have tried to produce spectro-photometric analogues of 67P nucleus using ternary mixtures of activated charcoal, tholins and water ice. Mixtures reproducing the individual spectrophotometric parameters (albedo, spectrum, phase reddening, phase curve, respectively) of 67P were obtained but the preparation of a unique mixture reproducing all these parameters together proved to be more challenging and has not been achieved yet. Nevertheless, these experiments allowed to put some constraints on the physical properties of 67P's surface dust, facilitating the interpretation of the data collected by OSIRIS (Feller et al., 2016; Hasselmann et al., 2017). Jost et al. (2017b) monitored the evolution of the phase curves of ice-dust mixtures during their sublimation, showing a reduction of scattering in the forward direction after the formation of the sublimation residues (Figure 15). The phase curve of 67P's surface is best matched by laboratory samples of highest porosities (sublimation residues of intra-mixtures, Jost et al., 2017b; Feller et al., 2016) and the strength of the phase slope may be indicative of the ice content (Hasselmann et al., 2017). The albedo of mixtures of carbon-rich dust with water ice particles measured by Jost et al. (2017a) indicate that the bright spots observed at the surface of 67P should be relatively dust free at small scale (lower than 5wt.\%). Moreover, the Vis-NIR spectra of different grain sizes of carbon-rich dust indicate that the darkening agent on 67P should not be dominated by particles in the 10-100 nm range (Jost et al., 2017a).

\subsection{Photometry of ice / basalt binary mixtures and implications for the poles of the Moon and Mercury.}

On planetary surfaces and cometary nuclei, ice is often found intimately mixed with the regolith at the grain level as indicated by spectral data (see for instance Neuman et al., 2013; Fillachione et al., 2016; Li et al., 2018 and other relevant references in Section 2.2.2). In such cases, one way to identify ice from remote is to look for its spectro- 
photometric signatures (e.g. high albedo or deep absorption bands at characteristic wavelengths). Unfortunately, it is not straightforward to interpret these signatures, since the reflectance of an ice-dust binary intimate mixture is a highly non-linear combination of the properties of its end-members (Clark and Lucey, 1984). Laboratory reflectance studies of intimate mixtures of ice and dust allow us to characterise their reflectance as a function of controlled parameters such as grain-size or ice-to-dust ratio. The results of such characterisations can serve as inputs and/or calibration for reflectance models, which are used to invert properties of planetary surfaces from their measured reflectance spectra.

Following the procedure detailed in Section 3.1.2, we produced intimate mixtures of SPIPA-A/-B and JSC1-AF lunar soil (ref JSC1-AF) in different concentrations: 0 wt.\%, 35 wt.\%-, 50 wt.\%- and 75 wt.\%- of water ice. Figure 16 shows the bidirectional reflectance and the VIS-NIR spectra of these samples. Bidirectional reflectance curves (Figure 16, a, b, c and d) show no significant photometric signature of ice for samples containing less than $50 \mathrm{wt} . \%$ of water ice - at phase angles smaller than $120^{\circ}$. Even samples containing up to $70 \mathrm{wt} . \%$ of water ice do not show specific signatures when the ice is present as relatively large ice particles (Figure 16, b and d). Therefore, when measured in the visible range and at phase angles lower than $120^{\circ}$, a $10 \%$-increase in the reflectance of a surface could imply a content of water ice intimately mixed within the soil of up to 50 wt.\%, depending on the size of the particles of ice.

Spectral reflectance measurements between 0.38 and $2.4 \mu \mathrm{m}$ (Figure 16, e and f) show a repetition of this trend at every wavelength of the continuum: only samples with more than $50 \mathrm{wt} . \%$ of water ice are distinguishable from ice-free samples. Outside the continuum, the characteristic absorption bands of water ice lower the detection threshold down to a 35 wt.\% water content. These laboratory measurements confirm that small amounts of fine dust can mask ice out of proportion to its mass fraction (Hapke, 1993). This is of special interest for cases such as the Moon, where small amounts of water ice if any- are expected to be mixed within the regolith in permanent shaded regions (PSRs) (ref moon). Some PSRs show a reflectance enhancement of about $10 \%$, which has been modelled to imply the presence of 6 to $14 \mathrm{wt} \%$ water ice within the regolith (Lucey et al., 2014). Nevertheless, the experiments that we have carried out show that such rises in reflectance can be caused by a 1:1 ice-to-regolith mass ratio under some conditions (Yoldi et al., 2015).

\subsection{Analogues of Martian icy surfaces}

We have simulated in the laboratory a variety of associations between ice and regolith and measured their reflectance to identify characteristic spectro-photometric signatures. We have mostly used JSC Mars-1 (Allen et al., 1997) as an analogue for the 
Martian bright red regolith but also darker and more pristine basaltic sands from Hawaii as analogues for dark basaltic regions (Pommerol et al., 2013).

Frost. Frost is the most likely form of water that can be found on the surface at low latitudes (Martínez et al., 2016). During cold nights, the regolith is likely to cold-trap atmospheric water, which sublimates in the early morning. Following the method explained in Section 3.1.3, we have induced the cold-trapping of water on various size-fractions of JSC Mars-1 (Figure 17a). Figure 17b, shows the spectra of the smallest size-distribution for three exposures of the samples to atmospheric water. The deposition of frost impacts the visible part of the reflectance spectrum in a much more significant way than the nearinfrared part of the spectrum. Pommerol et al. (2013) have also produced frosts on regolith analogues in a similar way to measure their visible bidirectional reflectance distribution function and have shown that the frosted surfaces display a very pronounced forward scattering behaviour.

Inter-mixtures. Inter-, or intimate, mixtures can, for instance, be the result of the redeposition of ice and regolith grains that wind has transported. Following the steps described in Section 3.1.2, we have produced intimate mixtures of JSC Mars-1 with 10wt\%, $35 \mathrm{wt} \%, 50 \mathrm{wt} \%$, and $75 \mathrm{wt} \%$ of water (Figure 17c). Figure 17d shows the spectra of the different ice concentrations, indicating that, contrary to the frost, the presence of water ice intimately mixed with the regolith has a larger impact on the near-infrared part of the spectrum than on its visible part.

Frozen soil. As explained in Section 3.1.3, we consider frozen soils as volumes of regolith saturated with iced water. Because of the limited amount of water vapour at the surface of Mars, this kind of mixture is likely restricted to the circum-polar areas. Figure 17e shows frozen soils prepared with four different size-fractions of JSC Mars-1 after 25 hours of sublimation. The sample on the bottom left has lost all of its surface ice, and the pure JSC Mars-1 is exposed. Figure $17 \mathrm{f}$ shows the temporal evolution of the spectra averaged over the area signalled by an orange rectangle on the image during 25 hours of sublimation. Initially saturated, the near-infrared spectra reveal the characteristics signatures of the JSC Mars-1, notably its red colour and hydration features as the layer of surface ice sublimates.

Pommerol et al. (2013) have measured the visible bidirectional reflectance distribution function of samples of frozen soils, including the ones shown in Figure 8 and observed that in addition to a strong decrease of the albedo, the addition of ice into the soil was responsible for strong changes in the geometry of the scattering. The presence of ice as a matrix in the bulk of the sample but not at the surface (Figure 8a) results in a strongly backscattering behaviour but clearly distinct from the one observed with the dry sample. In particular, the very intense opposition effect seen with the dry sample vanishes as ice is 
present within the sample and the maximum of reflectance is observed at higher emission angle on the backscattering side. As soon as a film of ice also covers the uppermost layer of grains (Figure 8b) a pronounced forward-scattering behaviour is observed. A specular peak is seen when the ice completely hides the topography of the grains (Figure 8c).

Beside studying the VIS-NIR reflectance spectra of our samples, we also simulate their colours as would be measured with the Colour and Stereo Surface Imaging System (CaSSIS, Thomas et al., 2017) on-board the ESA's Trace Gas Orbiter (TGO) using the instrument spectral response measured during the on-ground calibration campaign (Roloff et al., 2017). CaSSIS has four colour bands (475nm (BLU), 650nm (PAN), $850 \mathrm{~nm}$ (RED) and $950 \mathrm{~nm}(\mathrm{NIR})$ ) and is expected to complement the datasets collected by previous imagers of Mars with its high signal-to-noise ratio and the non-Sun-synchronous orbit of the TGO around Mars allowing us to look at the surface at various times of the day and the season. Figure $17 \mathrm{~g}$ and $\mathrm{h}$ show simulated CaSSIS colour-composite images corresponding to the data shown in Figure 17e (RED-PAN-BLU and NIR-RED-PAN, respectively).

\subsection{Measurements in the microwave domain and implications for comets, the Moon and icy satellites.}

Parameters measured by radars, such as time delay of the transmitted electromagnetic wave and its attenuation, and by radiometers, such as brightness temperature, depend on the electromagnetic properties of the sounded medium. Planetary surfaces, icy satellites and small bodies of the Solar System are considered as non-magnetic (unless metallic minerals are present in high proportion), so that the magnetic properties are equal to those of a vacuum and only the electrical properties, represented by the relative complex permittivity (hereafter permittivity), control the radiative transfer (Ulaby and Long, 2014). The real part, $\varepsilon^{\prime}$, represents how much energy from an electromagnetic wave is stored in the medium. The imaginary part, $\varepsilon "$, is proportional to the electrical conductivity of the medium and represents the loss by absorption. Permittivity varies with the frequency of measurement, the temperature, the bulk density, the porosity and the composition of the targeted material (Campbell and Ulrichs, 1969), so that its interpretation is challenging and requires to refer to laboratory measurements on analogue samples. However, the lack of standard procedures regarding the sample preparation and their characterization is not in favour of the reproducibility of the measurements and the trust towards the literature available to constrain the interpretation of radar data (Pettinelli et al., 2015). In response to that, we elaborated a programme of measurements in the $0.05-2$ $\mathrm{GHz}$ frequency range of electrical properties of pure water ice and inter-mixtures produced with the SPIPA setups. 
The measurements were performed at ambient temperature and at $243 \mathrm{~K}$. The intermixtures, prepared as described in Section 3.1.2 consisted of pure water ice particles mixed with refractory materials such as JSC-1A lunar regolith simulant, volcanic ashes from Etna, as well as tholins produced with the PAMPRE experimental setup (Szopa et al., 2006; Carrasco et al., 2009). We established reproducible procedures (Brouet et al., 2015) to measure their permittivity with porosities in the $30-95 \%$ range and dust-mass fractions in the $0-90 \%$ range. The porosity values of the samples were determined with a precision of less than $2 \%$ knowing the material density of the refractory materials, from literature and from measurements that we performed with a helium pycnometer Upyc-1200e-V5.04, and measuring their bulk density with a precision of less than $10 \%$. Bulk densities of the samples were measured before each permittivity measurement by weighing the filled sample holder, knowing the mass of the empty sample holder and its useful volume. The physical properties and dust-to-ice volumetric ratios of the samples, as well as the results of the measurements, are summarized in Table 6. The properties of the samples and the results are described in details by Brouet et al. (2015, 2016a, 2016b). The results show the absence of spectral dependence of $\varepsilon$ ' in the frequency range of interest. The porosity, temperature and dust-mass fraction dependences of $\varepsilon$ ' were determined. These results represent reference values for porous silicate-rich media, such as the regolith of the Moon, Mars, differentiated asteroids, as well as organic-rich media, such as Titan's surface. They also represent reference values for icy and porous silicate-rich media, such as cometary nuclei. A semi-empirical formula was derived from these results (equation 2 in Brouet et al., 2016b) which expresses the real part of the permittivity of a silicate-rich medium with a solid density of about 2.9 as a function of the porosity, the dust-mass fraction and the temperature for a frequency in the range of 0.05 to $2 \mathrm{GHz}$

In the framework of the Rosetta mission, we took advantage of this semi-empirical formula to interpret the $\varepsilon$ ' values determined for the cometary nucleus $67 /$ ChuryumovGerasimenko (hereafter 67P) from the radar sounder CONSERT and the permittivity probe SESAME-PP. From radiowaves at a centre-band frequency equal to $90 \mathrm{MHz}$, which propagated over a depth of one hundred of meters of the small lobe of 67P, Kofman et al. (2015) estimated a real part of the permittivity of about 1.27, whereas Lethuillier et al. (2016) estimated an upper limit of 2.45 for the first meter of material located at the final landing site of the Philae lander. Brouet et al. (2016b) showed that this difference may be caused by an increase of the porosity of the small lobe of 67P with depth from an upper limit of 55\% for the first meter below the Philae lander to values in the $67-86 \%$ range for the part of the small lobe sounded by CONSERT. 
The detection and estimation of the water ice volume fraction in the subsurface of Mars is a first-order goal for the science teams of the SHARAD and MARSIS radars (Bramson et al., 2015; Stuurman et al., 2016). We have conducted experimental dielectric measurements on pure JSC Mars-1 Martian soil simulant, SPIPA-B water ice particles and inter-mixtures with different dust-to-ice ratio and porosities in the frequency range of 50 $\mathrm{MHz}$ to $2 \mathrm{GHz}$. These results can be used to interpret the real part of the permittivity deduced from SHARAD and MARSIS radar echoes analyses to refine the estimates of water ice volume fraction in the Martian subsurface (Brouet et al., in press).

\subsection{Spectro-photometry of water ice particles containing pigmented microorganisms}

The putative emergence and sustainability of life in the internal salty water reservoirs of some satellites of the giant planets of the Solar System, such as Europa and Enceladus, foster the search for biosignatures on these objects (Hand et al., 2009; McKay et al., 2014). In particular, cryovolcanic eruptions expose samples of these internal reservoirs onto the surface of Enceladus (Scipioni et al., 2017), and possibly also of Europa (Sparks et al., 2017). Poch et al. (2017) studied the possibility to detect the spectral signature of microorganisms embedded in water ice particles, as it could be the case on active icy worlds. Liquid suspensions of Deinococcus radiodurans at various concentrations were nebulized into liquid nitrogen using the SPIPA-B setup (Section 3.1.1) to form ice particles made of an intra-mixture of water and bacteria. Then the ice particles were gradually deposited in the sample holders by sieving.

D. radiodurans are pigmented bacteria characterized by a steep spectral red slope between 0.5 and $0.6 \mu \mathrm{m}$ due to pigments and absorption bands of other biomolecules (proteins, lipids etc.) in the infrared. The reflectance spectra of the mixtures of bacteria with ice exhibit the pigments spectral red slope in the visible, but the absorption bands in the infrared are invisible because of the strong absorption of water ice at these wavelengths. The spectral red slope depends strongly on the concentration of bacteria and a detection threshold was determined. We also investigated the photometric phase curves of these samples, finding a dependence of the curvature of the phase curves with the concentration of bacteria in the ice and showing a strong phase reddening effect due to the presence of the biopigments. Finally, we studied how the sublimation of the top $5 \mathrm{~mm}$ of ice, and the consecutive concentration of the biotic material at the surface, may change the spectral reflectance of the samples. After sublimation, we found that the spectral red slope is increased by up to a factor of 2 , but the absorption bands of the biomolecules are 
surprisingly absent possibly because of the high porosity of the desiccated layer of bacteria and/or the dominating absorption of the water ice below this layer.

These results highlight the spectro-photometric properties of water ice particles containing pigments, but they do not permit to confirm their biogenic nature. The analysis of the spectral variations of the degree of circular polarization in the light scattered by biotic pigments may help to discriminate them from abiotic ones (Sparks et al., 2009).

\section{Discussion and perspectives}

\subsection{On the use of the Hapke model}

Most of the articles referenced in Section 4 mention and make use of the definitions and/or various versions of the Hapke model for the reflectance of surfaces (Section 2.2.1). In addition to the short discussions about the qualities and limitation of the model found in some of these articles, a few general comments on the nature and applicability of this model to laboratory data and remote sensing observations can be made. The Hapke model is wellsuited to fitting laboratory and planetary reflectance data, as it was intended to do. This is not particularly surprising as the empirical elements in the models have largely been introduced for that purpose. Therefore, the ability of the model to fit measured data cannot testify for its physical validity. It makes it, however, a very useful tool to interpolate, extrapolate, distribute and compare experimental reflectance data. This is the main use we have made of this model in our publications reporting on measured reflectance data. In the field of computer image simulation, mathematical functions such as spherical harmonics, splines or wavelets are widely used to interpolate measured BRDF data that are required for the simulation of 3D scenes. In the planetary science community, however, the Hapke model is widely used and implementations of the Hapke model are available in a variety of analysis and modelling softwares. The most appropriate way of exchanging and comparing reflectance data is thus by sharing the suite of Hapke coefficients that best fits the measured data.

By producing Hapke coefficients from a large variety of datasets, we have noticed some behaviours that are worth mentioning and discussing quickly. We could first confirm the strong correlation between the $b$ and c coefficients of the two-terms Henyey-Greenstein function classically used to describe the single scattering by an isolated particle (see Section 2.2.1). This correlation, referred to as the "hockey stick", was first mentioned by McGuire and Hapke (1995) and later described in detail by Hapke (2012). Unfortunately, noticing that this relation is verified for a variety of particles does not tell us anything about its cause, if it is an actual property of the grains or a feature of the model. 
The second general observation we make is that the "macroscopic roughness" parameter theta that represents in the model the mean slope angle of the facets of the samples at all scales larger than a few times the grain size, shows very little (if any) correlation with the actual roughness of the samples. This is very disappointing as distinguishing between the intrinsic photometric properties of the material that compose a planetary surface and large scale textural and structural effects is a requirement to be able to derive any meaningful quantitative information about the composition of a surface and its structure. Both types of properties are of major scientific interest. Good examples of the very limited correlation between retrieved values of the macroscopic roughness parameter and the texture of the actual samples can be seen in Figure 3 and Table 1 in Pommerol et al., 2013.

Because of the importance of this question and the doubts expressed in the literature about the validity of the physical approach followed by Hapke, we have decided to test this part of the Hapke model by confronting its results to results obtained by computer simulation using ray-tracing and fractal meshes. The ray-tracing model was implemented on Graphical Processing Unit for high computation performances, to allow us in the future to use the model with very complex surfaces (Gracia-Berná et al., 2016). Although the ray-tracing model can work with arbitrarily shapes surfaces, we have used it here with a fractal surface for which the mean slope angle can be calculated and compared to the macroscopic roughness parameter used in Hapke's model. Various surfaces were generated varying the mean slope angle between $0^{\circ}$ (smooth surface) and $50^{\circ}$ by steps of $10^{\circ}$. For each combination of incidence, emission, azimuth angle and surface roughness, parallel rays are cast along the incidence direction toward the surface. For each ray hitting a facet of the surface, the radiance contributed by this hit to the detector is calculated from a set of bidirectional reflectance data measured in the laboratory and interpolated, taking into account the local incidence, emission and phase angle at the location of the intersection. Summing the radiance from all facets of the surface seen by the detector provides the final value of radiance. Dividing this number by the number of incident rays intersecting the same surface allows us to retrieve its reflectance factor.

In parallel, we fit the same set of laboratory reflectance data using the Hapke model, while keeping the macroscopic roughness parameter constant and retrieve a set of Hapke parameters for the measured sample. We can then run the Hapke model from the set of parameters obtained from the fits of the experimental data but now varying the roughness parameter to produce phase curves for the same values of roughness as computed using the ray-tracing simulation. We have run the first test using laboratory measurements of a sample of powder produced from a small piece of the Allende carbonaceous chondrite. The 
measurements were performed with the PHIRE-2 instrument using the low phase angle setup described in Section 2.3. The comparison between the results of the two approaches shows a very good consistency in the effects of macroscopic roughness simulated using both methods (Figure 19).

So far, none of the results of our tests using the ray-tracing approach or of our laboratory measurements can indicate unambiguously a caveat in Hapke's model itself. And yet, Hapke parameters inverted from the measured data make little sense. It is possible that the inclusion of empirical parameters in the model prevents the physical interpretation of its parameters as argued by Shkuratov et al. (2012). The most likely problem with the application of the model to real samples however, is the large number of free parameters and the non-independence of the inversion of these parameters (i.e. varying different parameters in different ways can have similar effects on the reflectance curve calculated). This particular issue was well identified by Gunderson et al. (2006) in the analysis of the first reflectance measurements produced with the original PHIRE instrument.

This implies that irrelevant of the physical validity of the Hapke model, the complexity of natural particulate surfaces prevents the inversion of a physical model to derive meaningful quantitative properties from reflectance data alone. Independent constraints on some of the parameters involved, either related to the nature of the materials composing the surface or to its physical structure can certainly help in better constraining the inversion. Thus, the combination of multiple datasets acquired with different remote sensing methods and, if possible, a ground truth provided either by in-situ measurements or a returned sample is highly desirable. In the future, we would also like to test other models and compare their results with the Hapke model, starting with the series of models and codes developed at the University of Helsinki for both optical and radio wavelengths (Wilkman et al., 2015; Virkki and Muinonen, 2016; Markkanen et al., 2017). An integrated pipeline with an open web-based interface has recently been developed to facilitate the use of these codes by the remote sensing community (Pentillä et al., 2017).

\subsection{Distributing samples and data}

As briefly mentioned in Section 2.3, we have started distributing our icy samples by transporting the setups to produce them in the laboratories where the measurements will be conducted. Each new setup for icy samples production that we build is designed with the constraint that it must be transportable and easy to install. To date, we are able to pack all of our samples production setups and required equipment in a small truck, drive to various places in Europe and then re-install all the setups within half a day. Part of the 
equipment is also small and light enough to be shipped by airplane to more distant destinations. Alternatively, we publish with this article the detailed description of our setups (Figures 2, 3, and 4) and a list of all the components needed to build them (Supplementary Online Material 1). This should allow interested research groups to rebuild these setups and produce the same samples as we study here.

In the near future, it is one of our main objectives to complement the existing series of measurements by performing additional measurements using new techniques or by extending the spectral ranges or ranges of other parameters of our previous investigations. A comprehensive set of measurements on reference icy samples combining as many different techniques as possible will indeed be of prime importance for the analysis of existing and future multi-instrumental datasets collected by space missions.

In addition to the samples and their recipes, sharing the numerical spectrophotometric data measured in our laboratory has been one of our main objectives recently. We have chosen to start by distributing two types of data, bidirectional reflectance distribution functions (BRDF) and VIS-NIR reflectance spectra through two recently developed databases. We publish the bidirectional reflectance data measured with the PHIRE-2 instrument into the DACE (Data and Analysis Center for Exoplanets, https://dace.unige.ch/lossy/samplesearch/index) database developed by the University of Geneva in the framework of the NCCR PlanetS (Buchschacher et al., 2015). We publish the spectral data measured with the SCITEAS hyperspectral imager into the Bern icy Planetary Analogue Samples Spectroscopy (ByPASS) spectral database (https://www.sshade.eu/db/bypass), which is part of the Solid Spectroscopy Hosting Architecture of Databases and Expertise (SSHADE) database infrastructure (https://www.sshade.eu).

In both cases, numerical data are made available as standard ASCII tables accompanied by headers describing the data files in the XML format. As important as the measured data themselves, precise and quantitative descriptions of the samples are crucial for identifying and using the data. For both databases, we use the Solid Spectroscopy Data Model (SSDM) developed for SSHADE, which describes quantitatively the composition and structure of the samples as well as the physical conditions during the measurement.

As its acronym indicates, the DACE database is not only a repository for data but also provides powerful visualization tools and online analysis tools, such as model-fitting tools. We have already entered in this database all published data measured with the PHIRE-2 instrument. Visualisation tools are already online and a preliminary version of a tool to fit the Hapke model to the measured data is also implemented. This tool will be 
completed and improved in the near future. Mixing formulas in particular should be added to combine numerically the various materials available in the database. It is also our intention to provide other models (see Section 5.1) on the longer term. We also plan to add additional online calculations such as the hemispherical albedo integrated from BRDF data.

The ByPASS spectral database is the Bernese section of the European SSHADE database infrastructure (Schmitt et al., 2015) and thus benefits from all the recent developments made for this project especially in terms or sample description and search tools. We are currently ingesting our published data into the database, which has just opened.

\subsection{Planned improvements to the setups and samples; future work}

Most of the experimental work reported here is applicable to the surfaces of inner Solar System objects and comets such as 67P/CG that reach short heliocentric distances. Our photometric studies of pure micrometre-sized water ice (Jost et al., 2013, 2016) show interesting similarities between the behaviour of very fresh samples and the surface of Enceladus. However, the samples evolve very fast and rapidly lose any resemblance with the cold surface of Saturn's icy satellite. Allowing experimental work relevant for questions related to cold outer Solar System objects seems to be the logical next step in the development of the Planetary Ice Laboratory and is of high priority for the Planetary Imaging Group because of its involvement in two different types of projects:

- The future exploration of the icy Moons of Jupiter by ESA's JUICE and NASA's Europa Clipper missions. Our scientific interests in this topic are similar to the ones of our past and current work: produce reflectance data on plausible analogues as well as their electrical properties in the millimetre and submillimetre domains of wavelength, that help in interpreting the data and/or in planning the observations.

- The National Centre for Competence in Research (NCCR) PlanetS established in Switzerland. Its main scientific objectives are to study the origin and evolution of planetary systems, in particular by building bridges between the robotic exploration of the Solar System, astronomical observations of exoplanets and planetary disks, and theoretical and experimental studies in the laboratory. The scientific interactions within PlanetS and the will to build bridges between Solar System exploration and Exoplanets/disks observations resulted in the emergence of new projects around common scientific interests: 
1. The polarization of light reflected by mixtures of ice and dust with applications to the identification and characterization of water ice in proto-planetary disks observed in polarized light (Section 2.3).

2. The behaviour of $\mathrm{cm}$-sized agglomerates of ice and dust as they cross the ice line. Our sublimation experiments (Section 4.1) have shown that depending on the composition of the dust and the mixing mode of dust and ice, the outcome of the sublimation can be totally different, from a disruption of the icy pebble and dispersion of fine-grained dust to the preservation of the structural integrity of the pebble in the form of a porous and cohesive skeleton of dust. These outcomes have very different implications for the evolution of the proto-planetary disks and the formation of planetary embryos (See Section 2.1.3).

These two new scientific interests will drive the evolution of the Planetary Ice Laboratory in the next few years. Indeed, the studies intended require an extension of the technical capabilities of the laboratory to produce new icy samples in lower temperature and pressure environments, keep the samples at lower temperatures for longer durations and simulate space weathering by irradiating the samples with particles and energetic radiations.

\subsubsection{Samples}

\section{Narrower ice particles size distributions}

The SPIPA setups (Section 3.1) all produce samples with relatively broad size distributions. To study in more detail the effects of particle sizes, it will be necessary to produce narrower ice particle size distributions in a controlled and reproducible way. The technique used to sort and collect the particles may be different depending on the desired particle size range. For particles of several micrometers of diameter, they could be sorted mechanically in a sieving tower under a constant flux of liquid nitrogen. For particles of only few micrometers or sub-micrometric ice particles, sorting may be possibly achieved via gentle sedimentation or via vortexing in liquid nitrogen.

\section{Amorphous ice}

Working with thick samples of amorphous ice in the laboratory is challenging as the ice needs to be kept at a temperature lower than $120 \mathrm{~K}$ at all times to avoid crystallization. BarNun and Laufer (2003) have built a unique setup to produce cm-thick samples of porous gas-laden amorphous ice by repeatedly condensing water vapour onto cold-plate 
maintained at $80 \mathrm{~K}$ to produce $200 \mu \mathrm{m}$-thick layers of amorphous ice that are then scratched by a cold knife. The powder of $200 \mu \mathrm{m}$ particles produced in this process is stored in a sample container that is cooled down to $80 \mathrm{~K}$ before it is used for experiments (Laufer et al., 2013). We plan to develop a setup in which large amounts of amorphous ice can be produced and also mixed with mineral and organic refractory dust.

\section{Frost deposition in a controlled atmosphere or in near-vacuum}

We have recently started experimenting with frost deposition in the SCITEAS chamber (See Section 3.1.3, Figure 6 and Figure 7). Until now, all depositions of frost took place at ambient pressure. In the future, it will be necessary to complement this first set of measurements by varying the temperature of both the substrate and the atmosphere as well as the pressure and composition of the atmosphere. Indeed, the morphology of the frost is probably dependent on all these parameters.

\section{Higher dust-to-ice ratio}

A limitation to the applicability of the experiments conducted so far has been the low initial dust-to-ice ratio in all intra-mixture samples used. This is due to the limited availability of well-characterized tholins material and the difficulty to increase the amount of refractory matter in samples prepared as intra-mixtures when using the ultrasonic devices of the SPIPA-A and -B setups. The development of the new SPIPA-C setup now allows us to bypass this limitation and produce the samples with up to $50 \mathrm{wt}$. \% of dust required for application to comets and generally for outer proto-planetary disk material. Unfortunately, tholins are not currently produced at rates sufficient to be used for experiment with large amounts of dust. In addition, serious concerns exist about their toxicity. The use of terrestrial kerogens as the organic component in the dust offers significant advantages including availability and limited risks. A CI asteroid simulant based on the composition of the Orgueil meteorite and containing sub-bituminous coal is now commercialised by Deep Space Industries (http://deepspaceindustries.com/simulants/, Britt et al., 2017) and can be purchased in large amounts. We plan to test this material in our next set of experiments and compare the behaviour of ice mixed with the sub-bituminous coal used in this simulant with the behaviour previously observed with tholins.

\section{Salts}

Salts are observed in association with water ice at the surfaces of icy satellites (see Section 2.2.2). Their identification and characterization is of tremendous importance as they could provide insights about the conditions in the subsurface ocean if they indeed originate from the ocean and are not products of reactions between the ice and implanted species (see 
Section 2.1.3). We have started experimenting with the production of ice particles using the SPIPA setups and saline solutions. As an example, Figure 20 shows the results of operating the SPIPA-B setup with a solution of magnesium sulphate. As water cristallises, the magnesium and sulphate ions are concentrated at the periphery of the particles of ice and crystallise to form smaller and angular particles of $\mathrm{MgSO}_{4}$. The crystallized magnesium sulphate is probably hydrated but the exact hydration state could not be determined yet. The obtained texture visually shows similarities with inter-mixtures of SPIPA-B ice and contaminants (Section 3.1.2) and comparing the spectro-photometric properties of such samples will be instructive. The effect of such textures on the polarimetric phase curves of the samples measured with the POLICES setup (Section 2.3) should be particularly interesting.

\subsubsection{Setups and experiments}

\section{Improved simulation chamber: SCITEAS-2}

We are currently building a SCITEAS-2 setup to replace the one currently in use. Although SCITEAS has permitted a large number of investigations, we felt the need to improve the concept in several ways, listed in Table SCITEAS-2.

The new setup should allow us to better control and characterize the thermal environment of the samples. With this new configuration, a lot of interesting information could certainly be obtained by using a physical model to analyse the observed evolution of the samples (thickness, shape, temperature gradient) and chamber parameters (pressure, temperatures) during the course of the sublimation experiments. We plan to use the thermodynamical model develop for comets by Marboeuf et al., (2012) for that purpose. By fitting the model to measured data, we hope to derive values of the relevant physical parameters for the modelling of comets and other icy objects.

Simulate space weathering by irradiating the samples with particles and energetic radiations.

Most of the cold icy surfaces of the outer Solar System show much slower evolutions than the inner Solar System objects studied so far. As thermal metamorphism and sublimation are limited and in the absence of atmosphere, space weathering can dominate the evolution of the icy surfaces. We plan to study weathering by ultraviolet irradiation and electron bombardment directly in-situ in the new version of the SCITEAS setup and study weathering caused by ion bombardment by exchanging samples between the facilities of 
the Planetary Ice Laboratory and the MEFISTO setup. The MEFISTO (MEsskammer für FlugzeitInStrumente und Time-Of-Flight) is a large thermal vacuum chamber equipped with an ion source and operated by the mass spectrometry group of the space and planetary science department (Marti et al., 2001). It was mainly used in the past for the calibration of mass spectrometry flight instruments but a recent project aims at characterizing the process of sputtering of thick samples of ice by various ions (Galli et al., 2016, 2018) and by electrons (Galli et al., 2017). Such studies will have strong implications for the analysis of future data collected at Ganymede by the JUICE ESA mission and in particular by the Particle Environment Package (PEP) experiment (Barabash et al., 2013). The planetary imaging group contributes to this project by making the SPIPA setups available to produce the thick samples of porous ice used in the sputtering experiments. In addition, we have now built adapters to install the hyperspectral imaging system of SCITEAS on MEFISTO to follow in real-time the effects of the irradiation on the texture and spectral signatures of the surface of the sample.

\section{Multi-spectral setups for dielectric characterization of icy analogues}

In a context where the different space agencies express the will to develop permanent stations on the Moon and water ice reservoirs may be present in polar regions, the results presented in Section 4.5 regarding the microwave investigation represent an important dataset for the characterisation of the lunar subsurface in terms of ice content. Indeed, as shown in Section 4.5, the semi-empirical formula obtained from the experimental dataset allows us to also estimate the volume fraction of water ice in the lunar subsurface, which could be considered as an available resource supply for astronauts.

Two radiowave experiments are currently been built to explore the Jupiter's system in the next decades. ESA's JUICE spacecraft will be equipped with the RIME radar (Radar for Icy Moon Exploration, Bruzzone et al., 2013) and NASA's Europa Clipper will be equipped with the REASON radar (Grima et al., 2013), both instruments operating at 9 MHz. Their main objective is to study the structure and the composition of the subsurface of the icy Galilean satellites. The procedures developed for the preparation of the icy mixtures and the permittivity measurements summarized here should be taken into account as references for the preparation of methods dedicated to the data interpretation of these radar experiments, as well as for studies related to their performances, such as the estimation of their maximum penetration depth. In addition to experiments operating in the frequency range of planetary radars, the Planetary Ice laboratory is currently developping a dielectric measurement setup operating in the millimetre and submillmetre domains of wavelength, in collaboration with the THz-optics group of the University of Bern, with implications for the data interpretation of passive radiometers, in particular the 
Submillimetre Wave Instrument (SWI) on JUICE. Results have already been obtained on pure hydrated magnesium sulfates, as well as on inter-mixtures with SPIPA-B water ice particles (Brouet et al., 2017).

\section{Conclusion}

Water is the most abundant condensable compound in the Solar System and, as such, is present at or near the surface of many Solar System objects. It is also probably the most influent chemical species for the long-term evolution of proto-planetary disks and the resulting planetary systems. Its identification and characterization is thus a major objective of many planetary missions, equipped with a variety of remote sensing instruments, all the way from Mercury to Pluto. The Laboratory for Outflow Studies of Sublimating icy materials (LOSSy), also simply referred to as the Ice Laboratory has been developed since 2010 at the University of Bern to characterize the spectro-photometric properties and related physical properties of icy analogues of various Solar System surfaces. It complements the other activities of the Planetary Imaging Group by helping with the interpretation of optical remote sensing datasets and with the development of new remote sensing instruments. The three most significant achievements in terms of original technical developments are the low phase-angle setup for the PHIRE-2 gonio-radiometer, which allows us measuring the reflectivity of ice-bearing samples at near-zero phase angle, the VIS-NIR hyperspectral imaging system of the SCITEAS setup that allows us measuring spectral images of icy samples in-situ at very low temperature and pressure and the POLICES polarization-sensitive radio-goniometer which allows us to measure the polarization phase curve with very high accuracy.

But even more important than the development of these instruments, the biggest achievement of the project is certainly the definition and production of well-characterized and reproducible icy samples that will allow us to build progressively a consistent reference database of measurements over different spectral domains and using various techniques. In Bern, various spectro-photometric measurements have already been obtained on these samples. A number of results have been published in the scientific literature and all numerical data are currently ingested into public databases, together with some of our analysis tools. While additional spectro-photometric measurements will be conducted in Bern and while we continue expanding our collection of icy samples, we also distribute the samples to other groups specializing in other techniques and spectral domains. This can be done by moving the sample preparation setups, which we have already started doing, or by replicating the setups and protocols described in this publication. 
While our general methodology and philosophy have remained relatively unchanged throughout the project, the exact fields of application have been continuously evolving, reflecting the change in operating missions and new projects within the group. Indeed, the selection of planetary exploration missions and of their scientific instrumentation and the general scientific environment of the project are the main drivers for this evolution. Recently, work in the laboratory has been closely related to the development of the CaSSIS instrument for the Exomars/TGO mission, which was launched in March 2016. The strong involvement of different teams at the University of Bern in the development of three different instruments for ESA's JUICE mission to the Jovian system has also generated a new synergy in studying icy samples and a strong interest for the samples produced and characterized in the Planetary Ice Laboratory. New collaborations have already been initiated and start to provide results. The creation of the Centre for Space and Habitability at the University of Bern and the swiss National Centre for Competence in Research PlanetS have also strongly influenced the scientific thematic of the laboratory by imposing topics such as the definition of bio-signatures or the characterization of protoplanetary disks, broadening the range of applicability of the measurements performed in the laboratory and considerably enriching its scientific scope. New samples and instrumentation are currently being developed to reflect these evolutions. 


\section{References}

C.C. Allen, R.V. Morris, D.J. Lindstrom, M.M. Lindstrom, J.P. Lockwood, JSC Mars-1 Martian regolith simulant, in Lunar and Planetary Science Conference. Lunar and Planetary Inst. Technical Report, vol. 28, 1997

K. Altwegg, H. Balsiger, A. Bar-Nun, J. J. Berthelier, A. Bieler, P. Bochsler, C. Briois, U. Calmonte, M. Combi, J. De Keyser, P. Eberhardt, B. Fiethe, S. Fuselier, S. Gasc, T. I. Gombosi, K. C. Hansen, M. Hässig, A. Jäckel, E. Kopp, A. Korth, L. LeRoy, U. Mall, B. Marty, O. Mousis, E. Neefs, T. Owen, H. Rème, M. Rubin, T. Sémon, C.-Y. Tzou, H. Waite, P. Wurz, 67P/Churyumov-Gerasimenko, a Jupiter family comet with a high D/H ratio. Science 347, (2015). doi: 10.1126/science.1261952

J.D. Anderson, G. Schubert, Saturn's satellite Rhea is a homogeneous mix of rock and ice. Geophys. Res. Lett. 34, 02202 (2007). doi:10.1029/2006GL028100

A. Angstrom, The albedo of various surfaces of ground. Geografiska Annaler 7, 323-342 (1925)

T. Appéré, B. Schmitt, Y. Langevin, S. Douté, A. Pommerol, F. Forget, A. Spiga, B. Gondet, J.-P. Bibring, Winter and spring evolution of northern seasonal deposits on Mars from OMEGA on Mars Express. Journal of Geophysical Research (Planets) 116, 05001 (2011). doi:10.1029/2010JE003762

C. N. Banwell, Fundamentals of Molecular Spectroscopy, third edition, McGraw-Hill company (1983).

A. Bar-Nun, D. Laufer, First experimental studies of large samples of gas-laden amorphous "cometary" ices. Icarus 161, 157-163 (2003). doi:10.1016/S00191035(02)00016-7

S. Barabash, P. Wurz, P. Brandt, M. Wieser, M. Holmström, Y. Futaana, G. Stenberg, H. Nilsson, A. Eriksson, M. Tulej, A. Vorburger, N. Thomas, C. Paranicas, D.G. Mitchell, G. Ho, B.H. Mauk, D. Haggerty, J.H. Westlake, M. Fränz, N. Krupp, E. Roussos, E. Kallio, W. Schmidt, K. Szego, S. Szalai, K. Khurana, X. Jia, C. Paty, R.F. WimmerSchweingruber, B. Heber, A. Kazushi, M. Grande, H. Lammer, T. Zhang, S. McKennaLawlor, S.M. Krimigis, T. Sarris, D. Grodent, Particle Environment Package (PEP). European Planetary Science Congress 8, 2013-709 (2013)

M.A. Barucci, E. Dotto, A.C. Levasseur-Regourd, Space missions to small bodies: asteroids and cometary nuclei. A\&A Rev. 19, 48 (2011). doi:10.1007/s00159-011-0048-2

M.A. Barucci, F. Merlin, A. Guilbert, C. de Bergh, A. Alvarez-Candal, O. Hainaut, A. Doressoundiram, C. Dumas, T. Owen, A. Coradini, Surface composition and temperature of the TNO Orcus. A\&A 479, 13-16 (2008). doi:10.1051/0004-6361:20079079

P. Becerra, M.M. Sori, S. Byrne, Signals of astronomical climate forcing in the exposure topography of the North Polar Layered Deposits of Mars. Geophys. Res. Lett. 44, 62-70 (2017). doi:10.1002/2016GL071197 
P. Beck, E. Quirico, D. Sevestre, G. Montes-Hernandez, A. Pommerol, B. Schmitt, Goethite as an alternative origin of the $3.1 \mu \mathrm{m}$ band on dark asteroids. A\&A 526, 85 (2011). doi:10.1051/0004-6361/201015851

P. Beck, A. Pommerol, N. Thomas, B. Schmitt, F. Moynier, J.-A. Barrat, Photometry of meteorites. Icarus 218, 364-377 (2012). doi:10.1016/j.icarus.2011.12.005

M.J.S. Belton, J.W. Head III, A.P. Ingersoll, R. Greeley, A.S. McEwen, K.P. Klaasen, D. Senske, R. Pappalardo, G. Collins, A.R. Vasavada, R. Sullivan, D. Simonelli, P. Geissler, M.H. Carr, M.E. Davies, J. Veverka, P.J. Gierasch, D. Banfield, M. Bell, C.R. Chapman, C. Anger, R. Greenberg, G. Neukum, C.B. Pilcher, R.F. Beebe, J.A. Burns, F. Fanale, W. Ip, T.V. Johnson, D. Morrison, J. Moore, G.S. Orton, P. Thomas, R.A. West, Galileo's First Images of Jupiter and the Galilean Satellites. Science 274, 377-385 (1996). doi:10.1126/science.274.5286.377

J.-P. Bibring, Y. Langevin, F. Poulet, A. Gendrin, B. Gondet, M. Berthé, A. Sou $\triangleq$ ot, P. Drossart, M. Combes, G. Bellucci, V. Moroz, N. Mangold, B. Schmitt, OMEGA Team, S. Erard, O. Forni, N. Manaud, G. Poulleau, T. Encrenaz, T. Fouchet, R. Melchiorri, F. Altieri, V. Formisano, G. Bonello, S. Fonti, F. Capaccioni, P. Cerroni, A. Coradini, V. Kottsov, N. Ignatiev, D. Titov, L. Zasova, P. Pinet, C. Sotin, E. Hauber, H. Hoffman, R. Jaumann, U. Keller, R. Arvidson, J. Mustard, T. Duxbury, F. Forget, Perennial water ice identified in the south polar cap of Mars. Nature 428, 627-630 (2004).

doi:10.1038/nature02461

J. Brad Dalton, D.P. Cruikshank, R.N. Clark, Compositional analysis of Hyperion with the Cassini Visual and Infrared Mapping Spectrometer. Icarus 220, 752-776 (2012). doi:10.1016/j.icarus.2012.05.003

A.M. Bramson, S. Byrne, N.E. Putzig, S. Sutton, J.J. Plaut, T.C. Brothers, J. W. Holt, Widespread excess ice in Arcadia Planitia, Mars. Geophys. Res. Lett., 42, 6566-6574 (2015). doi:10.1002/2015GL064844.

D. Britt, S.D. Covey, C. Schultz, University of Central Florida / Deep Space Industries Asteroid Regolith Simulants, in AAS/Division for Planetary Sciences Meeting Abstracts \#49. AAS/Division for Planetary Sciences Meeting Abstracts, vol. 49, 2017, pp. 110-21

Y. Brouet, A.C. Levasseur-Regourd, P. Sabouroux, P. Encrenaz, N. Thomas, E. Heggy, W. Kofman, Permittivity measurements of porous matter in support of investigations of the surface and interior of 67P/Churyumov-Gerasimenko. A\&A 583, 39 (2015).

doi:10.1051/0004-6361/201526099

Y. Brouet, A.C. Levasseur-Regourd, P. Sabouroux, L. Neves, P. Encrenaz, O. Poch, A. Pommerol, N. Thomas, W. Kofman, A. Le Gall, V. Ciarletti, A. Hérique, A. Lethuillier, N. Carrasco, C. Szopa, A porosity gradient in 67P/C-G nucleus suggested from CONSERT and SESAME-PP results: an interpretation based on new laboratory permittivity measurements of porous icy analogues. MNRAS 462, 89-98 (2016a).

doi:10.1093/mnras/stw2151

Y. Brouet, L. Neves, P. Sabouroux, A.C. Levasseur-Regourd, O. Poch, P. Encrenaz, A. Pommerol, N. Thomas, W. Kofman, Characterization of the permittivity of controlled porous water ice-dust mixtures to support the radar exploration of icy bodies. Journal of Geophysical Research (Planets) 121, 2426-2443 (2016b). doi:10.1002/2016JE005045 
Y. Brouet, K. Jacob, A. Murk, R. Cerubini, A. Pommerol, N. Thomas, Dielectric properties of analogs of icy planetary surfaces in the $\mathrm{mm}$-submm domain, in relation with the JUICE mission. American Geophysical Union Fall Meeting, 11-15 Dec. 2017, New Orleans, United States.

Y. Brouet, P. Becerra, P. Sabouroux, A. Pommerol, N. Thomas, A laboratory-based dielectric model for the radar sounding of the Martian subsurface. Icarus, in press.

M.E. Brown, C.D. Koresko, G.A. Blake, Detection of Water Ice on Nereid. The Astrophys Journal Letters 508, 175-176 (1998). doi:10.1086/311741

M.E. Brown, E.L. Schaller, W.C. Fraser, Water Ice in the Kuiper Belt. The Astronomical Journal 143, 146 (2012). doi:10.1088/0004-6256/143/6/146

R.H. Brown, The Uranian satellites and Hyperion - New spectrophotometry and compositional implications. Icarus 56, 414-425 (1983). doi:10.1016/0019-1035(83)90163$\mathrm{X}$

R.H. Brown, R.N. Clark, Surface of Miranda - Identification of water ice. Icarus 58, $288-$ 292 (1984). doi:10.1016/0019-1035(84)90045-9

R.H. Brown, K.H. Baines, G. Bellucci, J.-P. Bibring, B.J. Buratti, F. Capaccioni, P. Cerroni, R.N. Clark, A. Coradini, D.P. Cruikshank, P. Drossart, V. Formisano, R. Jaumann, Y. Langevin, D.L. Matson, T.B. McCord, V. Mennella, E. Miller, R.M. Nelson, P.D. Nicholson, B. Sicardy, C. Sotin, The Cassini Visual And Infrared Mapping Spectrometer (Vims) Investigation. Space Sci. Rev. 115, 111-168 (2004). doi:10.1007/s11214-004-1453-x

R. Brunetto, M.A. Barucci, E. Dotto, G. Strazzulla, Ion Irradiation of Frozen Methanol, Methane, and Benzene: Linking to the Colors of Centaurs and Trans-Neptunian Objects. The Astrophys Journal 644, 646-650 (2006). doi:10.1086/503359

L. Bruzzone, J.J. Plaut, G. Alberti, D.D. Blankenship, F. Bovolo, B.A. Campbell, A. Ferro, Y. Gim, W. Kofman, G. Komatsu, W. McKinnon, G. Mitri, R. Orosei, G.W. Patterson, D. Plettemeier, R. Seu, RIME: Radar for Icy Moon Exploration. European Planetary Science Congress 8, 2013-744 (2013)

N. Buchschacher, D. Ségransan, S. Udry, R. D'iaz, Data and Analysis Center for Exoplanets, in Astronomical Data Analysis Software an Systems XXIV (ADASS XXIV), ed. by A.R. Taylor, E. Rosolowsky Astronomical Society of the Pacific Conference Series, vol. 495, 2015, p. 7

B.J. Buratti, J.A. Mosher, Comparative global albedo and color maps of the Uranian satellites. Icarus 90, 1-13 (1991). doi:10.1016/0019-1035(91)90064-Z

M.J. Campbell, J. Ulrichs, Electrical properties of rocks and their significance for lunar radar observations. J. Geophys. Res. 74, 5867-5881 (1969).

doi:10.1029/JB074i025p05867

H. Campins, K. Hargrove, N. Pinilla-Alonso, E.S. Howell, M.S. Kelley, J. Licandro, T. Mothé-Diniz, Y. Fernández, J. Ziffer, Water ice and organics on the surface of the asteroid 24 Themis. Nature 464, 1320- 1321 (2010). doi:10.1038/nature09029 
K.M. Cannon, J.F. Mustard, C.B. Agee, Evidence for a widespread basaltic breccia component in the martian low-albedo regions from the reflectance spectrum of Northwest Africa 7034. Icarus 252, 150-153 (2015). doi:10.1016/j.icarus.2015.01.016

R.W. Carlson, R.E. Johnson, M.S. Anderson, Sulfuric acid on Europa and the radiolytic sulfur cycle. Science 286, 97-99 (1999). doi:10.1126/science.286.5437.97

M. H. Carr, J. W. Head, Martian surface/near-surface water inventory: Sources, sinks, and changes with time. Geophysical Research Letters, Volume 42, Issue 3, pp. 726-732 (2015). doi: 10.1002/2014GL062464

N. Carrasco, I. Schmitz-Afonso, J.-Y. Bonnet, E. Quirico, R. Thissen, O. Dutuit, A. Bagag, O. Laprévote, A. Buch, A. Giulani, G. Adandé, F. Ouni, E. Hadamcik, C. Szopa, G. Cernogora, Chemical Characterization of Titan's Tholins: Solubility, Morphology and Molecular Structure Revisited. Journal of Physical Chemistry A 113, 11195-11203 (2009). doi:10.1021/jp904735q

S. Chandrasekhar, Radiative transfer. Oxford, Clarendon Press (1950).

R.N. Clark, P.G. Lucey, Spectral properties of ice-particulate mixtures and implications for remote sensing. I- Intimate mixtures. J. Geophys. Res. 89, 6341-6348 (1984).

doi:10.1029/JB089iB07p06341

R.N. Clark, G.A. Swayze, R. Wise, K.E. Livo, T.M. Hoefen, R.F. Kokaly, S.J. Sutley, A Digital Spectral Library for Planetary and Terrestrial Spectroscopy Analysis, in AAS/Division for Planetary Sciences Meeting Abstracts \#35. Bulletin of the American Astronomical Society, vol. 35, 2003, p. 948

G.D. Cody, H. Ade, C.M. O'D. Alexander, T. Araki, A. Butterworth, H. Fleckenstein, G. Flynn, M.K. Gilles, C. Jacobsen, A.L.D. Kilcoyne, K. Messenger, S.A. Sandford, T. Tyliszczak, A.J. Westphal, S. Wirick, H. Yabuta, Quantitative organic and light-element analysis of comet 81P/Wild 2 particles using $\mathrm{C}-, \mathrm{N}$-, and O-micro-XANES. Meteoritics and Planetary Science 43, 353-365 (2008). doi:10.1111/j.1945-5100.2008.tb00627.x

B.J.R. Davidsson, P.J. Gutiérrez, H. Sierks, C. Barbieri, P.L. Lamy, R. Rodrigo, D. Koschny, H. Rickman, H.U. Keller, J. Agarwal, M.F. A'Hearn, M.A. Barucci, J.-L. Bertaux, I. Bertini, D. Bodewits, G. Cremonese, V. Da Deppo, S. Debei, M. De Cecco, S.

Fornasier, M. Fulle, O. Groussin, C. Güttler, S.F. Hviid, W.-H. Ip, L. Jorda, J. Knollenberg, G. Kovacs, J.-R. Kramm, E. Kührt, M. Küppers, F. La Forgia, L.M. Lara, M. Lazzarin, J.J. Lopez Moreno, S. Lowry, S. Magrin, F. Marzari, H. Michalik, R. Moissl-Fraund, G. Naletto, N. Oklay, M. Pajola, C. Snodgrass, N. Thomas, C. Tubiana, J.-B. Vincent, Orbital elements of the material surrounding comet 67P/Churyumov-Gerasimenko. A\&A 583, 16 (2015). doi:10.1051/0004-6361/201525841

M.C. de Sanctis, E. Ammannito, A. Raponi, S. Marchi, T.B. McCord, H.Y. McSween, F. Capaccioni, M.T. Capria, F.G. Carrozzo, M. Ciarniello, A. Longobardo, F. Tosi, S. Fonte, M. Formisano, A. Frigeri, M. Giardino, G. Magni, E. Palomba, D. Turrini, F. Zambon, J.-P. Combe, W. Feldman, R. Jaumann, L.A. Mc-Fadden, C.M. Pieters, T. Prettyman, M. Toplis, C.A. Raymond, C.T. Russell, Ammoniated phyllosilicates with a likely outer Solar System origin on (1) Ceres. Nature 528, 241-244 (2015). doi:10.1038/nature16172

M.C. De Sanctis, F. Capaccioni, M. Ciarniello, G. Filacchione, M. Formisano, S. Mottola, A. Raponi, F. Tosi, D. Bockelée-Morvan, S. Erard, C. Leyrat, B. Schmitt, E. Ammannito, G. Arnold, M.A. Barucci, M. Combi, M.T. Capria, P. Cerroni, W.-H. Ip, E. Kuehrt, T.B. McCord, E. Palomba, P. Beck, E. Quirico, VIRTIS Team, G. Piccioni, G. Bellucci, M. 
Fulchignoni, R. Jaumann, K. Stephan, A. Longobardo, V. Mennella, A. Migliorini, J. Benkhoff, J.P. Bibring, A. Blanco, M. Blecka, R. Carlson, U. Carsenty, L. Colangeli, M. Combes, J. Crovisier, P. Drossart, T. Encrenaz, C. Federico, U. Fink, S. Fonti, P. Irwin, Y. Langevin, G. Magni, L. Moroz, V. Orofino, U. Schade, F. Taylor, D. Tiphene, G.P. Tozzi, N. Biver, L. Bonal, J.-P. Combe, D. Despan, E. Flamini, S. Fornasier, A. Frigeri, D. Grassi, M.S. Gudipati, F. Mancarella, K. Markus, F. Merlin, R. Orosei, G. Rinaldi, M. Cartacci, A. Cicchetti, S. Giuppi, Y. Hello, F. Henry, S. Jacquinod, J.M. Rees, R. Noschese, R. Politi, G. Peter, The diurnal cycle of water ice on comet 67P/ChuryumovGerasimenko. Nature 525, 500-503 (2015). doi:10.1038/nature14869

T. Denk, G. Neukum, T. Roatsch, C.C. Porco, J.A. Burns, G.G. Galuba, N. Schmedemann, P. Helfenstein, P.C. Thomas, R.J. Wagner, R.A. West, lapetus: Unique Surface Properties and a Global Color Dichotomy from Cassini Imaging. Science 327, 435 (2010). doi:10.1126/science.1177088

O.V. Dobrovolskii, E.A. Kaimakov, Surface phenomena in simulated cometary nuclei, in IAU Colloq. 39: Comets, Asteroids, Meteorites: Interrelations, Evolution and Origins, ed. by A.H. Delsemme, 1977, pp. 37-45

M. Dumont, O. Brissaud, G. Picard, B. Schmitt, J.-C. Gallet, Y. Arnaud, High-accuracy measurements of snow Bidirectional Reflectance Distribution Function at visible and NIR wavelengths - comparison with modelling results. Atmospheric Chemistry \& Physics 10 , 2507-2520 (2010)

C.M. Dundas, A.S. McEwen, M. Chojnacki, M.P. Milazzo, S. Byrne, J.N. McElwaine, A. Urso, Granular flows at recurring slope lineae on Mars indicate a limited role for liquid water. Nature Geoscience 10, 903-907 (2017). doi:10.1038/s41561-017-0012-5

M. Ebihara, N. Shirai, S. Sekimoto, T. Nakamura, A. Tsuchiyama, J. Matsuno, T. Matsumoto, M. Tanaka, M. Abe, A. Fujimura, Y. Ishibashi, Y. Karouji, T. Mukai, T. Okada, M. Uesugi, T. Yada, Chemical and mineralogical compositions of two grains recovered from asteroid Itokawa. Meteoritics and Planetary Science 50, 243-254 (2015). doi:10.1111/maps.12418

V.R. Eke, S.A. Bartram, D.A. Lane, D. Smith, L.F.A. Teodoro, Lunar polar craters - Icy, rough or just sloping? Icarus 241, 66-78 (2014). doi:10.1016/j.icarus.2014.06.021

M.R. El-Maarry, A. Pommerol, N. Thomas, Analysis of polygonal cracking patterns in chloride-bearing terrains on Mars: Indicators of ancient playa settings. Journal of Geophysical Research (Planets) 118, 2263-2278 (2013). doi:10.1002/2013JE004463

M.R. El-Maarry, A. Pommerol, N. Thomas, Desiccation of phyllosilicate-bearing samples as analog for desiccation cracks on Mars: Experimental setup and initial results. Planet. Space Sci. 111, 134-143 (2015). doi:10.1016/j.pss.2015.03.021

M.R. El-Maarry, W. Watters, N.K. McKeown, J. Carter, E. Noe Dobrea, J.L. Bishop, A. Pommerol, N. Thomas, Potential desiccation cracks on Mars: A synthesis from modeling, analogue-field studies, and global observations. Icarus 241, 248-268 (2014). doi:10.1016/j.icarus.2014.06.033

M.R. El-Maarry, W.A. Watters, Z. Yoldi, A. Pommerol, D. Fischer, U. Eggenberger, N. Thomas, Field investigation of dried lakes in western United States as an analogue to desiccation fractures on Mars. Journal of Geophysical Research (Planets) 120, 22412257 (2015). doi:10.1002/2015JE004895 
N. Engler, H.M. Schmid, C. Thalmann, A. Boccaletti, A. Bazzon, A. Baruffolo, J.L. Beuzit, R. Claudi, A. Costille, S. Desidera, K. Dohlen, C. Dominik, M. Feldt, T. Fusco, C. Ginski, D. Gisler, J.H. Girard, R. Gratton, T. Henning, N. Hubin, M. Janson, M. Kasper, Q. Kral, M. Langlois, E. Lagadec, F. Ménard, M.R. Meyer, J. Milli, D. Mouillet, J. Olofsson, A. Pavlov, J. Pragt, P. Puget, S.P. Quanz, R. Roelfsema, B. Salasnich, R. Siebenmorgen, E. Sissa, M. Suarez, J. Szulagyi, M. Turatto, S. Udry, F. Wildi, The HIP 79977 debris disk in polarized light. A\&A 607, 90 (2017). doi:10.1051/0004-6361/201730846

W.C. Feldman, T.H. Prettyman, S. Maurice, J.J. Plaut, D.L. Bish, D.T. Vaniman, M.T. Mellon, A.E. Metzger, S.W. Squyres, S. Karunatillake, W.V. Boynton, R.C. Elphic, H.O. Funsten, D.J. Lawrence, R.L. Tokar, Global distribution of near-surface hydrogen on Mars. Journal of Geophysical Research (Planets) 109, 09006 (2004).

doi:10.1029/2003JE002160

C. Feller, S. Fornasier, P.H. Hasselmann, A. Barucci, F. Preusker, F. Scholten, L. Jorda, A. Pommerol, B. Jost, O. Poch, M.R. EIMaary, N. Thomas, I. Belskaya, M. Pajola, H. Sierks, C. Barbieri, P.L. Lamy, D. Koschny, H. Rickman, R. Rodrigo, J. Agarwal, M. A'Hearn, J.-L. Bertaux, I. Bertini, S. Boudreault, G. Cremonese, V. Da Deppo, B.J.R. Davidsson, S. Debei, M. De Cecco, J. Deller, M. Fulle, A. Giquel, O. Groussin, P.J. Gutierrez, C. Güttler, M. Hofmann, S.F. Hviid, H. Keller, W.-H. Ip, J. Knollenberg, G. Kovacs, J.-R. Kramm, E. Kührt, M. Küppers, M.L. Lara, M. Lazzarin, C. Leyrat, J.J. Lopez Moreno, F. Marzari, N. Masoumzadeh, S. Mottola, G. Naletto, D. Perna, N. Oklay, X. Shi, C. Tubiana, J.-B. Vincent, Decimetre-scaled spectrophotometric properties of the nucleus of comet 67P/Churyumov-Gerasimenko from OSIRIS observations. MNRAS 462, $287-$ 303 (2016). doi:10.1093/mnras/stw2511

G. Filacchione, M.C. de Sanctis, F. Capaccioni, A. Raponi, F. Tosi, M. Ciarniello, P. Cerroni, G. Piccioni, M.T. Capria, E. Palomba, G. Bellucci, S. Erard, D. Bockelee-Morvan, C. Leyrat, G. Arnold, M.A. Barucci, M. Fulchignoni, B. Schmitt, E. Quirico, R. Jaumann, K. Stephan, A. Longobardo, V. Mennella, A. Migliorini, E. Ammannito, J. Benkhoff, J.P. Bibring, A. Blanco, M.I. Blecka, R. Carlson, U. Carsenty, L. Colangeli, M. Combes, M. Combi, J. Crovisier, P. Drossart, T. Encrenaz, C. Federico, U. Fink, S. Fonti, W.H. Ip, P. Irwin, E. Kuehrt, Y. Langevin, G. Magni, T. McCord, L. Moroz, S. Mottola, V. Orofino, U. Schade, F. Taylor, D. Tiphene, G.P. Tozzi, P. Beck, N. Biver, L. Bonal, J.-P. Combe, D. Despan, E. Flamini, M. Formisano, S. Fornasier, A. Frigeri, D. Grassi, M.S. Gudipati, D. Kappel, F. Mancarella, K. Markus, F. Merlin, R. Orosei, G. Rinaldi, M. Cartacci, A. Cicchetti, S. Giuppi, Y. Hello, F. Henry, S. Jacquinod, J.M. Reess, R. Noschese, R. Politi, G. Peter, Exposed water ice on the nucleus of comet 67P/Churyumov-Gerasimenko. Nature 529, 368-372 (2016a). doi:10.1038/nature16190

G. Filacchione, A. Raponi, F. Capaccioni, M. Ciarniello, F. Tosi, M.T. Capria, M.C. De Sanctis, A. Migliorini, G. Piccioni, P. Cerroni, M.A. Barucci, S. Fornasier, B. Schmitt, E. Quirico, S. Erard, D. Bockelee-Morvan, C. Leyrat, G. Arnold, V. Mennella, E. Ammannito, G. Bellucci, J. Benkhoff, J.P. Bibring, A. Blanco, M.I. Blecka, R. Carlson, U. Carsenty, L. Colangeli, M. Combes, M. Combi, J. Crovisier, P. Drossart, T. Encrenaz, C. Federico, U. Fink, S. Fonti, M. Fulchignoni, W.-H. Ip, P. Irwin, R. Jaumann, E. Kuehrt, Y. Langevin, G. Magni, T. McCord, L. Moroz, S. Mottola, E. Palomba, U. Schade, K. Stephan, F. Taylor, D. Tiphene, G.P. Tozzi, P. Beck, N. Biver, L. Bonal, J.-P. Combe, D. Despan, E. Flamini, M. Formisano, A. Frigeri, D. Grassi, M.S. Gudipati, D. Kappel, A. Longobardo, F. Mancarella, K. Markus, F. Merlin, R. Orosei, G. Rinaldi, M. Cartacci, A. Cicchetti, Y. Hello, F. Henry, S. Jacquinod, J.M. Reess, R. Noschese, R. Politi, G. Peter, Seasonal exposure 
of carbon dioxide ice on the nucleus of comet $67 \mathrm{P} / \mathrm{Churyumov}$-Gerasimenko. Science 354, 1563-1566 (2016b). doi:10.1126/science.aag3161

N.H. Fletcher, The Chemical Physics of Ice, Cambridge, UK: Cambridge University Press, 2009

S. Fornasier, E. Dotto, M.A. Barucci, C. Barbieri, Water ice on the surface of the large TNO 2004 DW. A\&A 422, 43-46 (2004). doi:10.1051/0004-6361:20048004

S. Fornasier, C. Lantz, M.A. Barucci, M. Lazzarin, Aqueous alteration on main belt primitive asteroids: Results from visible spectroscopy. Icarus 233, 163-178 (2014). doi:10.1016/j.icarus.2014.01.040

S. Fornasier, P.H. Hasselmann, M.A. Barucci, C. Feller, S. Besse, C. Leyrat, L. Lara, P.J. Gutierrez, N. Oklay, C. Tubiana, F. Scholten, H. Sierks, C. Barbieri, P.L. Lamy, R. Rodrigo, D. Koschny, H. Rickman, H.U. Keller, J. Agarwal, M.F. A'Hearn, J.-L. Bertaux, I. Bertini, G. Cremonese, V. Da Deppo, B. Davidsson, S. Debei, M. De Cecco, M. Fulle, O. Groussin, C. Güttler, S.F. Hviid, W. Ip, L. Jorda, J. Knollenberg, G. Kovacs, R. Kramm, E. Kührt, M. Küppers, F. La Forgia, M. Lazzarin, J.J. Lopez Moreno, F. Marzari, K.-D. Matz, H. Michalik, F. Moreno, S. Mottola, G. Naletto, M. Pajola, A. Pommerol, F. Preusker, X. Shi, C. Snodgrass, N. Thomas, J.-B. Vincent, Spectrophotometric properties of the nucleus of comet $67 \mathrm{P} /$ Churyumov-Gerasimenko from the OSIRIS instrument onboard the ROSETTA spacecraft. A\&A 583, 30 (2015). doi:10.1051/0004-6361/201525901

S. Fornasier, S. Mottola, H.U. Keller, M.A. Barucci, B. Davidsson, C. Feller, J.D.P. Deshapriya, H. Sierks, C. Barbieri, P.L. Lamy, R. Rodrigo, D. Koschny, H. Rickman, M. A'Hearn, J. Agarwal, J.-L. Bertaux, I. Bertini, S. Besse, G. Cremonese, V. Da Deppo, S. Debei, M. De Cecco, J. Deller, M.R. El-Maarry, M. Fulle, O. Groussin, P.J. Gutierrez, C. Güttler, M. Hofmann, S.F. Hviid, W.-H. Ip, L. Jorda, J. Knollenberg, G. Kovacs, R. Kramm, E. Kührt, M. Küppers, M.L. Lara, M. Lazzarin, J.J.L. Moreno, F. Marzari, M. Massironi, G. Naletto, N. Oklay, M. Pajola, A. Pommerol, F. Preusker, F. Scholten, X. Shi, N. Thomas, I. Toth, C. Tubiana, J.-B. Vincent, Rosetta's comet 67P/ChuryumovGerasimenko sheds its dusty mantle to reveal its icy nature. Science $354,1566-1570$ (2016). doi:10.1126/science.aag2671

N. Fray, B. Schmitt, Sublimation of ices of astrophysical interest: A bibliographic review. Planet. Space Sci. 57, 2053-2080 (2009). doi:10.1016/j.pss.2009.09.011

A. Galli, A. Vorburger, A. Pommerol, P. Wurz, B. Jost, O. Poch, Y. Brouet, M. Tulej, N. Thomas, Surface charging of thick porous water ice layers relevant for ion sputtering experiments. Planet. Space Sci. 126, 63-71 (2016). doi:10.1016/j.pss.2016.03.016

A. Galli, A. Vorburger, P. Wurz, M. Tulej, Sputtering of water ice films: A re-assessment with singly and doubly charged oxygen and argon ions, molecular oxygen, and electrons. Icarus 291, 36-45 (2017). doi:10.1016/j.icarus.2017.03.018

A. Galli, A. Vorburger, P. Wurz, R. Cerubini, and M. Tulej, First experimental data of sulphur ions sputtering water ice, Icarus 312 (2018), 1-6, DOI:

10.1016/j.icarus.2018.04.029.

A. Garenne, G. Montes-Hernandez, P. Beck, B. Schmitt, O. Brissaud, A. Pommerol, Gassolid carbonation as a possible source of carbonates in cold planetary environments. Planet. Space Sci. 76, 28-41 (2013). doi:10.1016/j.pss.2012.11.005 
E. Georget, R. Abdeddaim, P. Sabouroux, A quasi-universal method to measure the electromagnetic characteristics of usual materials in the microwave range, C. R. Phys., 15, 448-457, 2014.

A. Gracia-Berná, A. Pommerol, N. Thomas, R. Marschall, Z. Yoldi, B. Jost, O. Poch, Photometry of fractal meshes for applications to large-scale rough planetary surfaces, in GPU Technology Conference. GPU Technology Conference, 2016

O. Grasset, M.K. Dougherty, A. Coustenis, E.J. Bunce, C. Erd, D. Titov, M. Blanc, A. Coates, P. Drossart, L.N. Fletcher, H. Hussmann, R. Jaumann, N. Krupp, J.-P. Lebreton, O. Prieto-Ballesteros, P. Tortora, F. Tosi, T. Van Hoolst, JUpiter ICy moons Explorer (JUICE): An ESA mission to orbit Ganymede and to characterise the Jupiter system. Planet. Space Sci. 78, 1-21 (2013). doi:10.1016/j.pss.2012.12.002

C. Grima, D.M. Schroeder, D.D. Blankenship, Identifying Surface Characteristics with an Ice Penetrating Radar Sounder at Europa: Potential for Landing Site Selection, in Lunar and Planetary Science Conference. Lunar and Planetary Inst. Technical Report, vol. 44, 2013, p. 2980

E. Grün, H. Kochan, K.J. Seidensticker, Laboratory simulation, a tool for comet research. Geophys. Res. Lett. 18, 245-248 (1991). doi:10.1029/90GL02522

A. Guilbert-Lepoutre, Survival of Amorphous Water Ice on Centaurs. The Astronomical Journal 144, 97 (2012). doi:10.1088/0004-6256/144/4/97

K. Gunderson, N. Thomas, Polarimetric NIR reflectance measurements of regolith simulants at zero phase angle. Planet. Space Sci. 56, 1925-1938 (2008).

doi:10.1016/j.pss.2008.09.004

K. Gunderson, N. Thomas, J.A. Whitby, First measurements with the Physikalisches Institut Radiometric Experiment (PHIRE). Planet. Space Sci. 54, 1046-1056 (2006). doi:10.1016/j.pss.2005.12.020

K. Gunderson, B. Lüthi, P. Russell, N. Thomas, Visible/NIR photometric signatures of liquid water in Martian regolith simulant. Planet. Space Sci. 55, 1272-1282 (2007). doi:10.1016/j.pss.2007.03.004

B. Gundlach, J. Blum, The Stickiness of Micrometer-sized Water-ice Particles. The Astrophys Journal 798, 34 (2015). doi:10.1088/0004-637X/798/1/34

B. Gundlach, Y.V. Skorov, J. Blum, Outgassing of icy bodies in the Solar System - I. The sublimation of hexagonal water ice through dust layers. Icarus 213, 710-719 (2011a). doi:10.1016/j.icarus.2011.03.022

B. Gundlach, S. Kilias, E. Beitz, J. Blum, Micrometer-sized ice particles for planetaryscience experiments - I. Preparation, critical rolling friction force, and specific surface energy. Icarus 214, 717-723 (2011b). doi:10.1016/j.icarus.2011.05.005

K.P. Hand, R.W. Carlson, Europa's surface color suggests an ocean rich with sodium chloride. Geophys. Res. Lett. 42, 3174-3178 (2015). doi:10.1002/2015GL063559

K.P. Hand, C.F. Chyba, J.C. Priscu, R.W. Carlson, K.H. Nealson, Astrobiology and the Potential for Life on Europa, ed. by R.T. Pappalardo, W.B. McKinnon, K.K. Khurana 2009, p. 589 
R. Hanel, B. Conrath, F.M. Flasar, V. Kunde, W. Maguire, J.C. Pearl, J. Pirraglia, R. Samuelson, D.P. Cruikshank, D. Gautier, P.J. Gierasch, L. Horn, C. Ponnamperuma, Infrared observations of the Saturnian system from Voyager 2. Science $215,544-548$ (1982). doi:10.1126/science.215.4532.544

G.B. Hansen, T.B. McCord, Amorphous and crystalline ice on the Galilean satellites: A balance between thermal and radiolytic processes. Journal of Geophysical Research (Planets) 109, 01012 (2004). doi:10.1029/2003JE002149

G.B. Hansen, E.C. Hollenbeck, K. Stephan, S.K. Apple, E.-J.Z. Shin-White, Water ice abundance and $\mathrm{CO} 2$ band strength on the saturnian satellite Phoebe from Cassini/VIMS observations. Icarus 220, 331-338 (2012). doi:10.1016/j.icarus.2012.05.004

A. Hanslmeier, Water in the Universe, Astrophysics and Space Science Library, Volume 368. ISBN 978-90-481-9983-9. Springer Science+Business Media B.V. (2011). doi: 10.1007/978-90-481-9984-6

B. Hapke, Bidirectional reflectance spectroscopy. 1. Theory. J. Geophys. Res. 86, 45714586 (1981)

B. Hapke, Bidirectional reflectance spectroscopy. III - Correction for macroscopic roughness. Icarus 59, 41-59 (1984). doi:10.1016/0019-1035(84)90054-X

B. Hapke, Theory of reflectance and emittance spectroscopy 1993

B. Hapke, Bidirectional Reflectance Spectroscopy. 5. The Coherent Backscatter Opposition Effect and Anisotropic Scattering. Icarus 157, 523-534 (2002).

doi:10.1006/icar.2002.6853

B. Hapke, Theory of Reflectance and Emittance Spectroscopy 2005, p. 469

B. Hapke, Bidirectional reflectance spectroscopy. 6 . Effects of porosity. Icarus 195, 918926 (2008). doi:10.1016/j.icarus.2008.01.003

B. Hapke, Bidirectional reflectance spectroscopy 7 . The single particle phase function hockey stick relation. Icarus 221, 1079-1083 (2012). doi:10.1016/j.icarus.2012.10.022

B. Hapke, Comment on "A critical assessment of the Hapke photometric model" by Y. Shkuratov et al. J. Quant. Spec. Radiat. Transf. 116, 184-190 (2013).

doi:10.1016/j.jqsrt.2012.11.002

B. Hapke, E. Wells, Bidirectional reflectance spectroscopy. II - Experiments and observations. J. Geophys. Res. 86, 3055-3060 (1981). doi:10.1029/JB086iB04p03055

J.K. Harmon, M.A. Slade, Radar mapping of Mercury - Full-disk images and polar anomalies. Science 258, 640-643 (1992). doi:10.1126/science.258.5082.640

P.H. Hasselmann, M.A. Barucci, S. Fornasier, C. Feller, J.D.P. Deshapriya, M.

Fulchignoni, B. Jost, H. Sierks, C. Barbieri, P.L. Lamy, R. Rodrigo, D. Koschny, H.

Rickman, M. A'Hearn, J.-L. Bertaux, I. Bertini, G. Cremonese, V. Da Deppo, B.

Davidsson, S. Debei, M. De Cecco, J. Deller, M. Fulle, R.W. Gaskell, O. Groussin, P.J.

Gutierrez, C. Güttler, M. Hofmann, S.F. Hviid, W.-H. Ip, L. Jorda, H.U. Keller, J.

Knollenberg, G. Kovacs, R. Kramm, E. Kührt, M. Küppers, M.L. Lara, M. Lazzarin, J.J. 
Lopez-Moreno, F. Marzari, S. Mottola, G. Naletto, N. Oklay, A. Pommerol, N. Thomas, C. Tubiana, J.-B. Vincent, The opposition effect of 67P/Churyumov-Gerasimenko on postperihelion Rosetta images. MNRAS 469, 550-567 (2017). doi:10.1093/mnras/stx1834 E. Hauber, D. Reiss, M. Ulrich, F. Preusker, F. Trauthan, M. Zanetti, H. Hiesinger, R. Jaumann, L. Johansson, A. Johnsson, S. Van Gaselt, M. Olvmo, Landscape evolution in Martian mid-latitude regions: insights from analogous periglacial landforms in Svalbard. In: Balme, M., A. Bargery, C. Gallagher, S. Guta (eds). Martian Geomorphology. Geological Society, London. Special Publications: 356. 111-131 (2011).

E. Heggy, P. Paillou, G. Ruffie, J.M. Malezieux, F. Costard, G. Grandjean, On Water Detection in the Martian Subsurface Using Sounding Radar. Icarus 154, 244-257 (2001). doi:10.1006/icar.2001.6717

E. Heggy, E.M. Palmer, W. Kofman, S.M. Clifford, K. Righter, A. Hérique, Radar properties of comets: Parametric dielectric modeling of Comet 67P/ChuryumovGerasimenko. Icarus 221, 925-939 (2012). doi:10.1016/j.icarus.2012.09.023

P. Helfenstein, J. Veverka, D. McCarthy, P. Lee, J. Hillier, Large quasi-circular features beneath frost on Triton. Science 255, 824-826 (1992). doi:10.1126/science.255.5046.824

L.G. Henyey, J.L. Greenstein, Diffuse radiation in the Galaxy. The Astrophys Journal 93, 70-83 (1941). doi:10.1086/144246

M. Hilchenbach, J. Kissel, Y. Langevin, C. Briois, H. von Hoerner, A. Koch, R. Schulz, J. Silén, K. Altwegg, L. Colangeli, H. Cottin, C. Engrand, H. Fischer, A. Glasmachers, E. Grün, G. Haerendel, H. Henkel, H. Höfner, K. Hornung, E.K. Jessberger, H. Lehto, K. Lehto, F. Raulin, L. Le Roy, J. Rynö, W. Steiger, T. Stephan, L. Thirkell, R. Thomas, K. Torkar, K. Varmuza, K.-P. Wanczek, N. Altobelli, D. Baklouti, A. Bardyn, N. Fray, H. Krüger, N. Ligier, Z. Lin, P. Martin, S. Merouane, F.R. Orthous-Daunay, J. Paquette, C. Revillet, S. Siljeström, O. Stenzel, B. Zaprudin, Comet 67P/Churyumov-Gerasimenko: Close-up on Dust Particle Fragments. The Astrophys Journal Letters 816, 32 (2016). doi:10.3847/2041-8205/816/2/L32

H.-W. Hsu, F. Postberg, Y. Sekine, T. Shibuya, S. Kempf, M. Horanyi, A. Juhasz, N. Altobelli, K. Suzuki, Y. Masaki, T. Kuwatani, S. Tachibana, S.-I. Sirono, G. MoragasKlostermeyer, R. Srama, Ongoing hydrothermal activities within Enceladus. Nature 519, 207-210 (2015). doi:10.1038/nature14262

S.R. Hudson, S.G. Warren, R.E. Brandt, T.C. Grenfell, D. Six, Spectral bidirectional reflectance of Antarctic snow: Measurements and parameterization. Journal of Geophysical Research (Atmospheres) 111, 18106 (2006). doi:10.1029/2006JD007290

L. less, D.J. Stevenson, M. Parisi, D. Hemingway, R.A. Jacobson, J.I. Lunine, F. Nimmo, J.W. Armstrong, S.W. Asmar, M. Ducci, P. Tortora, The Gravity Field and Interior Structure of Enceladus. Science 344, 78-80 (2014). doi:10.1126/science.1250551

R. Jaumann, K. Stephan, G.B. Hansen, R.N. Clark, B.J. Buratti, R.H. Brown, K.H. Baines, S.F. Newman, G. Bellucci, G. Filacchione, A. Coradini, D.P. Cruikshank, C.A. Griffith, C.A. Hibbitts, T.B. McCord, R.M. Nelson, P.D. Nicholson, C. Sotin, R. Wagner, Distribution of icy particles across Enceladus' surface as derived from Cassini-VIMS measurements. Icarus 193, 407-419 (2008). doi:10.1016/j.icarus.2007.09.013

D.C. Jewitt, J. Luu, Crystalline water ice on the Kuiper belt object (50000) Quaoar. Nature 432, 731-733 (2004). doi:10.1038/nature03111 
T.V. Johnson, J.I. Lunine, Saturn's moon Phoebe as a captured body from the outer Solar System. Nature 435, 69-71 (2005). doi:10.1038/nature03384

K.L. Jones, R.E. Arvidson, E.A. Guinness, S.L. Bragg, S.D. Wall, C.E. Carlston, D.G. Pidek, One Mars year- Viking lander imaging observations. Science 204, 799-806 (1979). doi:10.1126/science.204.4395.799

B. Jost, B. Gundlach, A. Pommerol, J. Oesert, S.N. Gorb, J. Blum, N. Thomas, Micrometer-sized ice particles for planetary-science experiments - II. Bidirectional reflectance. Icarus 225, 352-366 (2013). doi:10.1016/j.icarus.2013.04.007

B. Jost, A. Pommerol, O. Poch, B. Gundlach, M. Leboeuf, M. Dadras, J. Blum, N. Thomas, Experimental characterization of the opposition surge in fine-grained water-ice and high albedo ice analogs. Icarus 264, 109-131 (2016).

doi:10.1016/j.icarus.2015.09.020

B. Jost, A. Pommerol, O. Poch, Z. Yoldi, S. Fornasier, P.H. Hasselmann, C. Feller, N. Carrasco, C. Szopa, N. Thomas, Bidirectional reflectance and VIS-NIR spectroscopy of cometary analogues under simulated space conditions. Planet. Space Sci. 145, 14-27 (2017a). doi:10.1016/j.pss.2017.07.009

B. Jost, A. Pommerol, O. Poch, Y. Brouet, S. Fornasier, N. Carrasco, C. Szopa, N. Thomas, Bidirectional reflectance of laboratory cometary analogues to interpret the spectrophotometric properties of the nucleus of comet 67P/Churyumov-Gerasimenko. Planet. Space Sci. 148, 1-11 (2017b). doi:10.1016/j.pss.2017.09.009

S. Kaasalainen, M. Kaasalainen, T. Mielonen, J. Suomalainen, J.I. Peltoniemi, J. Näränen, Optical properties of snow in backscatter. Journal of Glaciology 52, 574-584 (2006). doi:10.3189/172756506781828421

J.S. Kargel, Cryovolcanism on the icy satellites. Earth Moon and Planets 67, 101-113 (1994). doi:10.1007/BF00613296

K.K. Khurana, R.T. Pappalardo, N. Murphy, T. Denk, The origin of Ganymede's polar caps. Icarus 191, 193-202 (2007). doi:10.1016/j.icarus.2007.04.022

H.H. Kieffer, P.R. Christensen, T.N. Titus, $\mathrm{CO} 2$ jets formed by sublimation beneath translucent slab ice in Mars' seasonal south polar ice cap. Nature 442, 793-796 (2006). doi:10.1038/nature04945

W. Kofman, A. Herique, Y. Barbin, J.-P. Barriot, V. Ciarletti, S. Clifford, P. Edenhofer, C. Elachi, C. Eyraud, J.-P. Goutail, E. Heggy, L. Jorda, J. Lasue, A.-C. Levasseur-Regourd, E. Nielsen, P. Pasquero, F. Preusker, P. Puget, D. Plettemeier, Y. Rogez, H. Sierks, C. Statz, H. Svedhem, I. Williams, S. Zine, J. Van Zyl, Properties of the 67P/ChuryumovGerasimenko interior revealed by CONSERT radar. Science 349(2) (2015). doi:10.1126/science.aab0639

R.F. Kokaly, R.N. Clark, G.A. Swayze, K.E. Livo, T.M. Hoefen, N.C. Pearson, R.A. Wise, W.M. Benzel, H.A. Lowers, R.L. Driscoll, USGS Spectral Library Version 7. U.S. Geological Survey Data Series 1035, 61 (2017). doi:https://doi.org/10.3133/ds1035 
N.I. Kömle, G. Kargl, K. Thiel, K. Seiferlin, Thermal properties of cometary ices and sublimation residuals including organics. Planet. Space Sci. 44, 675-689 (1996). doi:10.1016/0032-0633(96)00043-8

K.J. Kossacki, W.J. Markiewicz, Comet 67P/CG: Influence of the sublimation coefficient on the temperature and outgassing. Icarus 224, 172-177 (2013).

doi:10.1016/j.icarus.2013.02.012

K.J. Kossacki, T. Spohn, A. Hagermann, E. Kaufmann, E. Kührt, Comet 67P/ChuryumovGerasimenko: Hardening of the sub-surface layer. Icarus 260, 464-474 (2015). doi:10.1016/j.icarus.2015.07.024

M. Küppers, H.U. Keller, E. Kührt, M.F. A'Hearn, K. Altwegg, R. Bertrand, H. Busemann, M.T. Capria, L. Colangeli, B. Davidsson, P. Ehrenfreund, J. Knollenberg, S. Mottola, A. Rathke, P. Weiss, M. Zolensky, E. Akim, A. Basilevsky, E. Galimov, M. Gerasimov, O. Korablev, I. Lomakin, M. Marov, M. Martynov, M. Nazarov, A. Zakharov, L. Zelenyi, A. Aronica, A.J. Ball, C. Barbieri, A. Bar-Nun, J. Benkhoff, J. Biele, N. Biver, J. Blum, D. Bockelée-Morvan, O. Botta, J.-H. Bredehöft, F. Capaccioni, S. Charnley, E. Cloutis, H. Cottin, G. Cremonese, J. Crovisier, S.A. Crowther, E.M. Epifani, F. Esposito, A.C. Ferrari, F. Ferri, M. Fulle, J. Gilmour, F. Goesmann, N. Gortsas, S.F. Green, O. Groussin, E. Grün, P.J. Gutiérrez, P. Hartogh, T. Henkel, M. Hilchenbach, T.-M. Ho, G. Horneck, S.F. Hviid, W.-H. Ip, A. Jäckel, E. Jessberger, R. Kallenbach, G. Kargl, N.I. Kömle, A. Korth, K. Kossacki, C. Krause, H. Krüger, Z.-Y. Li, J. Licandro, J.J. Lopez-Moreno, S.C. Lowry, I. Lyon, G. Magni, U. Mall, I. Mann, W. Markiewicz, Z. Martins, M. Maurette, U. Meierhenrich, V. Mennella, T.C. Ng, L.R. Nittler, P. Palumbo, M. Pätzold, D. Prialnik, M. Rengel, H. Rickman, J. Rodriguez, R. Roll, D. Rost, A. Rotundi, S. Sandford, M. Schönbächler, H. Sierks, R. Srama, R.M. Stroud, S. Szutowicz, C. Tornow, S. Ulamec, M. Wallis, W. Waniak, P. Weissman, R. Wieler, P. Wurz, K.L. Yung, J.C. Zarnecki, Triple F a comet nucleus sample return mission. Experimental Astronomy 23, 809-847 (2009). doi:10.1007/s10686-008-9115-8

M. Küppers, L. O'Rourke, D. Bockelée-Morvan, V. Zakharov, S. Lee, P. von Allmen, B. Carry, D. Teyssier, A. Marston, T. Müller, J. Crovisier, M.A. Barucci, R. Moreno, Localized sources of water vapour on the dwarf planet (1)Ceres. Nature 505, 525-527 (2014). doi:10.1038/nature12918

H. Kurokawa, M. Sato, M. Ushioda, T. Matsuyama, R. Moriwaki, J.M. Dohm, T. Usui, Evolution of water reservoirs on Mars: Constraints from hydrogen isotopes in martian meteorites. Earth and Planetary Science Letters 394, 179-185 (2014).

doi:10.1016/j.epsl.2014.03.027

P. Lacerda, S. Fornasier, E. Lellouch, C. Kiss, E. Vilenius, P. Santos-Sanz, M. Rengel, T. Müller, J. Stansberry, R. Duffard, A. Delsanti, A. Guilbert-Lepoutre, The Albedo-Color Diversity of Transneptunian Objects. The Astrophys Journal Letters 793, 2 (2014). doi:10.1088/2041-8205/793/1/L2

M. Lambrechts, A. Johansen, Rapid growth of gas-giant cores by pebble accretion. A\&A 544, 32 (2012). doi:10.1051/0004-6361/201219127

M. Lambrechts, A. Johansen, Forming the cores of giant planets from the radial pebble flux in protoplanetary discs. A\&A 572, 107 (2014). doi:10.1051/0004-6361/201424343

M. Lambrechts, A. Johansen, A. Morbidelli, Separating gas-giant and ice-giant planets by halting pebble accretion. A\&A 572, 35 (2014). doi:10.1051/0004-6361/201423814 
P.L. Lamy, I. Toth, Y.R. Fernandez, H.A. Weaver, The sizes, shapes, albedos, and colors of cometary nuclei, ed. by M.C. Festou, H.U. Keller, H.A. Weaver 2004, pp. 223-264 D. Laufer, A. Bar-Nun, I. Pat-El, R. Jacovi, Experimental studies of ice grain ejection by massive gas flow from ice and implications to Comets, Triton and Mars. Icarus 222, 73 80 (2013). doi:10.1016/j.icarus.2012.10.030

D.J. Lawrence, W.C. Feldman, J.O. Goldsten, S. Maurice, P.N. Peplowski, B.J. Anderson, D. Bazell, R.L. McNutt, L.R. Nittler, T.H. Prettyman, D.J. Rodgers, S.C. Solomon, S.Z. Weider, Evidence for Water Ice Near Mercury's North Pole from MESSENGER Neutron Spectrometer Measurements. Science 339, 292 (2013). doi:10.1126/science.1229953

A. Lefort, P.S. Russell, N. Thomas, Scalloped terrains in the Peneus and Amphitrites Paterae region of Mars as observed by HiRISE. Icarus 205, 259-268 (2010). doi:10.1016/j.icarus.2009.06.005

A. Lethuillier, A. Le Gall, M. Hamelin, W. Schmidt, K.J. Seidensticker, R. Grard, V. Ciarletti, S. Caujolle-Bert, H.-H. Fischer, R. Trautner, Electrical properties and porosity of the first meter of the nucleus of 67P/Churyumov-Gerasimenko. As constrained by the Permittivity Probe SESAME-PP/Philae/Rosetta. A\&A 591, 32 (2016). doi:10.1051/0004$6361 / 201628304$

H.F. Levison, K.A. Kretke, M.J. Duncan, Growing the gas-giant planets by the gradual accumulation of pebbles. Nature 524, 322-324 (2015a). doi:10.1038/nature14675

H.F. Levison, K.A. Kretke, K.J. Walsh, W.F. Bottke, Growing the terrestrial planets from the gradual accumulation of sub-meter sized objects. Proceedings of the National Academy of Science 112, 14180-14185 (2015b). doi:10.1073/pnas.1513364112

J.-Y. Li, M.F. A'Hearn, M.J.S. Belton, C.J. Crockett, T.L. Farnham, C.M. Lisse, L.A. McFadden, K.J. Meech, J.M. Sunshine, P.C. Thomas, J. Veverka, Deep Impact photometry of Comet 9P/Tempel 1. Icarus 187, 41- 55 (2007).

doi:10.1016/j.icarus.2006.09.018

J.-Y. Li, S. Besse, M.F. A'Hearn, M.J.S. Belton, D. Bodewits, T.L. Farnham, K.P. Klaasen, C.M. Lisse, K.J. Meech, J.M. Sunshine, P.C. Thomas, Photometric properties of the nucleus of Comet 103P/Hartley 2. Icarus 222, 559-570 (2013).

doi:10.1016/j.icarus.2012.11.001

S. Li, P.G. Lucey, R.E. Milliken, P.O. Hayne, E.Fisher, J.-P. Williams, D.M. Hurley, R.C. Elphic, Direct evidence of surface exposed water ice in the lunar polar regions. PNAS 115 (36), 8907-8912 (2018). doi: https://doi.org/10.1073/pnas.1802345115

J. Licandro, H. Campins, M. Kelley, K. Hargrove, N. Pinilla-Alonso, D. Cruikshank, A.S. Rivkin, J. Emery, (65) Cybele: detection of small silicate grains, water-ice, and organics. A\&A 525, 34 (2011). doi:10.1051/0004-6361/201015339

P. J. Linstrom, W. G. Mallard, NIST Chemistry WebBook, NIST Standard Reference Database Number 69, National Institute of Standards and Technology, Gaithersburg MD, 20899 (2005) doi: 10.18434/T4D303

L. Lliboutry, The origin of penitents. Journal of Glaciology 2, 331-338 (1954) 
N. Ligier, F. Poulet, J. Carter, R. Brunetto, F. Gourgeot, VLT/SINFONI Observations of Europa: New Insights into the Surface Composition. The Astronomical Journal 151, 163 (2016). https://doi.org/10.3847/0004-6256/151/6/163

K. Lodders, Abundances and Condensation Temperatures of the Elements. Meteoritics and Planetary Science Supplement 38 (2003)

A. Lucas, O. Aharonson, C. Deledalle, A.G. Hayes, R. Kirk, E. Howington-Kraus, Insights into Titan's geology and hydrology based on enhanced image processing of Cassini RADAR data. Journal of Geophysical Research (Planets) 119, 2149-2166 (2014). doi:10.1002/2013JE004584

P.G. Lucey, G.A. Neumann, M.A. Riner, E. Mazarico, D.E. Smith, M.T. Zuber, D.A. Paige, D.B. Bussey, J.T. Cahill, A. McGovern, P. Isaacson, L.M. Corley, M.H. Torrence, H.J. Melosh, J.W. Head, E. Song, The global albedo of the Moon at $1064 \mathrm{~nm}$ from LOLA. Journal of Geophysical Research (Planets) 119, 1665-1679 (2014). doi:10.1002/2013JE004592

N. Mangold, S. Maurice, W.C. Feldman, F. Costard, F. Forget, Spatial relationships between patterned ground and ground ice detected by the Neutron Spectrometer on Mars. Journal of Geophysical Research (Planets) 109, 08001 (2004).

doi:10.1029/2004JE002235

U. Marboeuf, B. Schmitt, J.-M. Petit, O. Mousis, N. Fray, A cometary nucleus model taking into account all phase changes of water ice: amorphous, crystalline, and clathrate. A\&A 542, 82 (2012). doi:10.1051/0004-6361/201118176

J. Markkanen, T. Väisänen, A. Penttilä, K. Muinonen, Light scattering and absorption by space weathered planetary bodies: Novel numerical solution, in AAS/Division for Planetary Sciences Meeting Abstracts \#49. AAS/Division for Planetary Sciences Meeting Abstracts, vol. 49, 2017, pp. 110-42

A. Marti, R. Schletti, P. Wurz, P. Bochsler, Calibration facility for solar wind plasma instrumentation. Review of Scientific Instruments 72, 1354-1360 (2001).

doi: $10.1063 / 1.1340020$

G.M. Martinez, E. Fischer, N.O. Rennó, E. Sebastián, O. Kemppinen, N. Bridges, C.S. Borlina, P.-Y. Meslin, M. Genzer, A.-H. Harri, A. Vicente-Retortillo, M. Ramos, M. de la Torre Juárez, F. Gómez, J. Gómez-Elvira, Likely frost events at Gale crater: Analysis from MSL/REMS measurements. Icarus 280, 93-102 (2016).

doi:10.1016/j.icarus.2015.12.004

B. Marty, The origins and concentrations of water, carbon, nitrogen and noble gases on Earth. Earth and Planetary Science Letters 313, 56-66 (2012).

doi:10.1016/j.epsl.2011.10.040

B. Marty, K. Altwegg, H. Balsiger, A. Bar-Nun, D. V. Bekaert, J. J. Berthelier, A. Bieler, C. Briois, U. Calmonte, M. Combi, J. De Keyser, B. Fiethe, S. A. Fuselier, S. Gasc, T. I. Gombosi, K. C. Hansen, M. Hässig, A. Jäckel, E. Kopp, A. Korth, L. LeRoy, U. Mall, O. Mousis, T. Owen, H. Rème, M. Rubin, T. Sémon, C.-Y. Tzou, H. Waite, P. Wurz, Xenon isotopes in 67P/Churyumov-Gerasimenko show that comets contributed to Earth's atmosphere. Science 356, 1069-1072 (2017). 
C. McCarthy, R.F. Cooper, D.L. Goldsby, W.B. Durham, S.H. Kirby, Transient and steady state creep response of ice I and magnesium sulfate hydrate eutectic aggregates. Journal of Geophysical Research (Planets) 116, 04007 (2011). doi:10.1029/2010JE003689

T.B. McCord, G.B. Hansen, R.N. Clark, P.D. Martin, C.A. Hibbitts, F.P. Fanale, J.C. Granahan, M. Segura, D.L. Matson, T.V. Johnson, R.W. Carlson, W.D. Smythe, G.E. Danielson, Non-water-ice constituents in the surface material of the icy Galilean satellites from the Galileo near-infrared mapping spectrometer investigation. J. Geophys. Res. 103, 8603-8626 (1998a). doi:10.1029/98JE00788

T.B. McCord, G.B. Hansen, F.P. Fanale, R.W. Carlson, D.L. Matson, T.V. Johnson, W.D. Smythe, J.K. Crow-ley, P.D. Martin, A. Ocampo, C.A. Hibbitts, J.C. Granahan, Salts on Europa's Surface Detected by Galileo's Near Infrared Mapping Spectrometer. Science 280, 1242 (1998b). doi:10.1126/science.280.5367.1242

A.F. McGuire, B.W. Hapke, An experimental study of light scattering by large, irregular particles. Icarus 113, 134-155 (1995). doi:10.1006/icar.1995.1012

C.P. McKay, A.D. Anbar, C. Porco, P. Tsou, Follow the Plume: The Habitability of Enceladus. Astrobiology 14, 352-355 (2014). doi:10.1089/ast.2014.1158

D.S. McKay, J.L. Carter, W.W. Boles, C.C. Allen, J.H. Allton, JSC-1: A new lunar regolith simulant, in Lunar and Planetary Science Conference. Lunar and Planetary Inst.

Technical Report, vol. 24, 1993

F. Merlin, A. Guilbert, C. Dumas, M.A. Barucci, C. de Bergh, P. Vernazza, Properties of the icy surface of the TNO 136108 (2003 EL61). A\&A 466, 1185-1188 (2007).

doi:10.1051/0004-6361:20066866

T. Michikami, A.M. Nakamura, N. Hirata, The shape distribution of boulders on Asteroid 25143 Itokawa: Comparison with fragments from impact experiments. Icarus 207, 277284 (2010). doi:10.1016/j.icarus.2009.10.008

G. Mie, Beitrage zur Optik truber Medien, speziell kolloidaler Metallosungen. Annalen der Physik 330, 377-445 (1908). doi:10.1002/andp.19083300302

R.S. Miller, G. Nerurkar, D.J. Lawrence, Enhanced hydrogen at the lunar poles: New insights from the detection of epithermal and fast neutron signatures. Journal of Geophysical Research (Planets) 117, 11007 (2012). doi:10.1029/2012JE004112

R.E. Milliken, J.F. Mustard, F. Poulet, D. Jouglet, J.-P. Bibring, B. Gondet, Y. Langevin, Hydration state of the Martian surface as seen by Mars Express OMEGA: 2. H2O content of the surface. Journal of Geophysical Research (Planets) 112, 08-07 (2007).

doi:10.1029/2006JE002853

S. T. F. Möhlmann, Adsorption Water-Related Potential Chemical and Biological Processes in the Upper Martian Surface. Astrobiology Vol. 5, No. 6, 770-777 (2005). doi: 10.1089/ast.2005.5.770

D.T.F. Möhlmann, M. Niemand, V. Formisano, H. Savijarvi, P. Wolkenberg, Fog phenomena on Mars. Planet. Space Sci. 57, 1987-1992 (2009).

doi:10.1016/j.pss.2009.08.003 
M.H. Moore, R.L. Hudson, Far-infrared spectral studies of phase changes in water ice induced by proton irradiation. The Astrophys Journal 401, 353-360 (1992).

doi:10.1086/172065

A. Morbidelli, B. Bitsch, A. Crida, M. Gounelle, T. Guillot, S. Jacobson, A. Johansen, M. Lambrechts, E. Lega, Fossilized condensation lines in the Solar System protoplanetary disk. Icarus 267, 368-376 (2016). doi:10.1016/j.icarus.2015.11.027

J. Mouginot, A. Pommerol, P. Beck, W. Kofman, S.M. Clifford, Dielectric map of the Martian northern hemisphere and the nature of plain filling materials. Geophys. Res. Lett. 39, 02202 (2012). doi:10.1029/2011GL050286

K. Muinonen, Coherent backscattering of light by complex random media of spherical scatterers: numerical solution. Waves in Random Media 14, 365-388 (2004).

doi:10.1088/0959-7174/14/3/010

K. Muinonen, M.I. Mishchenko, J.M. Dlugach, E. Zubko, A. Penttilä, G. Videen, Coherent Backscattering Verified Numerically for a Finite Volume of Spherical Particles. The Astrophys Journal 760, 118 (2012). doi:10.1088/0004-637X/760/2/118

S. Murchie, R. Arvidson, P. Bedini, K. Beisser, J.-P. Bibring, J. Bishop, J. Boldt, P. Cavender, T. Choo, R.T. Clancy, E.H. Darlington, D. Des Marais, R. Espiritu, D. Fort, R. Green, E. Guinness, J. Hayes, C. Hash, K. Heffernan, J. Hemmler, G. Heyler, D. Humm, J. Hutcheson, N. Izenberg, R. Lee, J. Lees, D. Lohr, E. Malaret, T. Martin, J.A. McGovern, P. McGuire, R. Morris, J. Mustard, S. Pelkey, E. Rhodes, M. Robinson, T. Roush, E. Schaefer, G. Seagrave, F. Seelos, P. Silverglate, S. Slavney, M. Smith, W.-J. Shyong, K. Strohbehn, H. Taylor, P. Thompson, B. Tossman, M. Wirzburger, M. Wolff, Compact Reconnaissance Imaging Spectrometer for Mars (CRISM) on Mars Reconnaissance Orbiter (MRO). Journal of Geophysical Research (Planets) 112, 05-03 (2007). doi:10.1029/2006JE002682

A.M. Nakamura, T. Michikami, N. Hirata, A. Fujiwara, R. Nakamura, M. Ishiguro, H. Miyamoto, H. Demura, K. Hiraoka, T. Honda, C. Honda, J. Saito, T. Hashimoto, T. Kubota, Impact process of boulders on the surface of asteroid 25143 Itokawa - fragments from collisional disruption. Earth, Planets, and Space 60, 7-12 (2008).

doi:10.1186/BF03352756

T. Nakamura, T. Noguchi, M. Tanaka, M.E. Zolensky, M. Kimura, A. Tsuchiyama, A. Nakato, T. Ogami, H. Ishida, M. Uesugi, T. Yada, K. Shirai, A. Fujimura, R. Okazaki, S.A. Sandford, Y. Ishibashi, M. Abe, T. Okada, M. Ueno, T. Mukai, M. Yoshikawa, J. Kawaguchi, Itokawa Dust Particles: A Direct Link Between S-Type Asteroids and Ordinary Chondrites. Science 333, 1113 (2011). doi:10.1126/science.1207758

D. Nakashima, N.T. Kita, T. Ushikubo, T. Noguchi, T. Nakamura, J.W. Valley, Oxygen three-isotope ratios of silicate particles returned from asteroid Itokawa by the Hayabusa spacecraft: A strong link with equilibrated LL chondrites. Earth and Planetary Science Letters 379, 127-136 (2013). doi:10.1016/j.epsl.2013.08.009

A. Nathues, M. Hoffmann, M. Schaefer, L. Le Corre, V. Reddy, T. Platz, E.A. Cloutis, U. Christensen, T. Kneissl, J.-Y. Li, K. Mengel, N. Schmedemann, T. Schaefer, C.T. Russell, D.M. Applin, D.L. Buczkowski, M.R.M. Izawa, H.U. Keller, D.P. O'Brien, C.M. Pieters, C.A. Raymond, J. Ripken, P.M. Schenk, B.E. Schmidt, H. Sierks, M.V. Sykes, G.S. Thangjam, J.-B. Vincent, Sublimation in bright spots on (1) Ceres. Nature 528, 237-240 (2015). doi:10.1038/nature15754 
G.A. Neumann, J.F. Cavanaugh, X. Sun, E.M. Mazarico, D.E. Smith, M.T. Zuber, D. Mao, D.A. Paige, S.C. Solomon, C.M. Ernst, O.S. Barnouin, Bright and Dark Polar Deposits on Mercury: Evidence for Surface Volatiles. Science 339, 296 (2013).

doi:10.1126/science.1229764

S.F. Newman, B.J. Buratti, R.H. Brown, R. Jaumann, J. Bauer, T. Momary, Photometric and spectral analysis of the distribution of crystalline and amorphous ices on Enceladus as seen by Cassini. Icarus 193, 397-406 (2008). doi:10.1016/j.icarus.2007.04.019

A.M. Nicolson, G.F Ross, Measurement of the intrinsic properties of materials by timedomain techniques, IEEE Trans. Instr. and Meas., 19, 377-382, 1970.

F. Nimmo, J.R. Spencer, R.T. Pappalardo, M.E. Mullen, Shear heating as the origin of the plumes and heat flux on Enceladus. Nature 447, 289-291 (2007).

doi:10.1038/nature05783

J.A. Nuth III, S.B. Charnley, N.M. Johnson, Chemical Processes in the Interstellar Medium: Source of the Gas and Dust in the Primitive Solar Nebula, ed. by D.S. Lauretta, H.Y. McSween 2006, pp. 147-167

K.I. Oberg, R.T. Garrod, E.F. van Dishoeck, H. Linnartz, Formation rates of complex organics in UV irradiated $\mathrm{CH} 3 \mathrm{OH}$-rich ices. I. Experiments. A\&A 504, 891-913 (2009). doi:10.1051/0004-6361/200912559

A. Oehler, G. Neukum, Visible and near IR albedo measurements of ice/dust mixtures. Geophys. Res. Lett. 18, 253-256 (1991). doi:10.1029/91GL00174

N. Oklay, J.-B. Vincent, S. Fornasier, M. Pajola, S. Besse, B.J.R. Davidsson, L.M. Lara, S. Mottola, G. Naletto, H. Sierks, A.M. Barucci, F. Scholten, F. Preusker, A. Pommerol, N. Masoumzadeh, M. Lazzarin, C. Barbieri, P.L. Lamy, R. Rodrigo, D. Koschny, H. Rickman, M.F. A'Hearn, J.-L. Bertaux, I. Bertini, D. Bodewits, G. Cremonese, V. Da Deppo, S. Debei, M. De Cecco, M. Fulle, O. Groussin, P.J. Gutiérrez, C. Güttler, I. Hall, M. Hofmann, S.F. Hviid, W.-H. Ip, L. Jorda, H.U. Keller, J. Knollenberg, G. Kovacs, J.-R. Kramm, E. Kührt, M. Küppers, Z.-Y. Lin, J.J. Lopez Moreno, F. Marzari, F. Moreno, X. Shi, N. Thomas, I. Toth, C. Tubiana, Variegation of comet 67P/Churyumov-Gerasimenko in regions showing activity. A\&A 586, 80 (2016). doi:10.1051/0004-6361/201527369

T. Okuchi, Hydrogen Partitioning into Molten Iron at High Pressure: Implications for Earth's Core. Science 278, 1781 (1997). doi:10.1126/science.278.5344.1781

D.A. Paige, M.A. Siegler, J.K. Harmon, G.A. Neumann, E.M. Mazarico, D.E. Smith, M.T. Zuber, E. Harju, M.L. Delitsky, S.C. Solomon, Thermal Stability of Volatiles in the North Polar Region of Mercury. Science 339, 300 (2013). doi:10.1126/science.1231106

P. Paillou, M. Schuster, S. Tooth, T. Farr, A. Rosenqvist, S. Lopez, J.-M. Malezieux, Mapping of a major paleodrainage system in eastern Libya using orbital imaging radar: The Kufrah River. Earth and Planetary Science Letters 277, 327-333 (2009).

doi:10.1016/j.epsl.2008.10.029

E. D. Pallik, Handbook of optical constants of solids II. Boston: Academic Press (1991).

A. Penttilä, T. Väisänen, J. Markkanen, J. Martikainen, M. Gritsevich, K. Muinonen, Multiple scattering modeling pipeline for spectroscopy and photometry of airless Solar 
System objects, in AAS/Division for Planetary Sciences Meeting Abstracts \#49. AAS/Division for Planetary Sciences Meeting Abstracts, vol. 49, 2017, pp. 208-02

E. Pettinelli, B. Cosciotti, F. Di Paolo, S.E. Lauro, E. Mattei, R. Orosei, G. Vannaroni, Dielectric properties of Jovian satellite ice analogs for subsurface radar exploration: A review. Reviews of Geophysics 53, 593-641 (2015). doi:10.1002/2014RG000463

C. Pilorget, M. Vincendon, F. Poulet, A radiative transfer model to simulate light scattering in a compact granular medium using a Monte-Carlo approach: Validation and first applications. Journal of Geophysical Research (Planets) 118, 2488-2501 (2013). doi:10.1002/2013JE004465

N. Pinilla-Alonso, J. Licandro, R. Gil-Hutton, R. Brunetto, The water ice rich surface of (145453) 2005 RR43:a case for a carbon-depleted population of TNOs? A\&A 468, 25-28 (2007). doi:10.1051/0004-6361:20077294

K.M. Pitman, B.J. Buratti, J.A. Mosher, Disk-integrated bolometric Bond albedos and rotational light curves of saturnian satellites from Cassini Visual and Infrared Mapping Spectrometer. Icarus 206, 537-560 (2010). doi:10.1016/j.icarus.2009.12.001

O. Poch, A. Pommerol, B. Jost, N. Carrasco, C. Szopa, N. Thomas, Sublimation of icetholins mixtures: A morphological and spectro-photometric study. Icarus 266, 288-305 (2015). doi:10.1016/j.icarus.2015.12.017

O. Poch, A. Pommerol, B. Jost, N. Carrasco, C. Szopa, N. Thomas, Sublimation of water ice mixed with silicates and tholins: Evolution of surface texture and reflectance spectra, with implications for comets. Icarus 267, 154-173 (2016).

doi:10.1016/j.icarus.2015.12.017

O. Poch, J. Frey, I. Roditi, A. Pommerol, B. Jost, N. Thomas, Remote Sensing of Potential Biosignatures from Rocky, Liquid, or Icy (Exo)Planetary Surfaces. Astrobiology 17, 231-252 (2017). doi:10.1089/ast.2016.1523

A. Pommerol, B. Schmitt, P. Beck, O. Brissaud, Water sorption on martian regolith analogs: Thermodynamics and near-infrared reflectance spectroscopy. Icarus 204, 114 136 (2009). doi:10.1016/j.icarus.2009.06.013

A. Pommerol, G. Portyankina, N. Thomas, K.-M. Aye, C.J. Hansen, M. Vincendon, Y. Langevin, Evolution of south seasonal cap during Martian spring: Insights from highresolution observations by HiRISE and CRISM on Mars Reconnaissance Orbiter. Journal of Geophysical Research (Planets) 116, 08007 (2011a). doi:10.1029/2010JE003790

A. Pommerol, N. Thomas, M. Affolter, G. Portyankina, B. Jost, K. Seiferlin, K.-M. Aye, Photometry and bulk physical properties of Solar System surfaces icy analogs: The Planetary Ice Laboratory at University of Bern. Planet. Space Sci. 59, 1601-1612 (2011b). doi:10.1016/j.pss.2011.07.009

A. Pommerol, T. Appéré, G. Portyankina, K.-M. Aye, N. Thomas, C.J. Hansen, Observations of the northern seasonal polar cap on Mars III: CRISM/HiRISE observations of spring sublimation. Icarus 225, 911-922 (2013a). doi:10.1016/j.icarus.2012.08.039

A. Pommerol, N. Thomas, B. Jost, P. Beck, C. Okubo, A.S. McEwen, Photometric properties of Mars soils analogs. Journal of Geophysical Research (Planets) 118, 20452072 (2013b). doi:10.1002/jgre.20158 
A. Pommerol, N. Thomas, M.R. El-Maarry, M. Pajola, O. Groussin, A.-T. Auger, N. Oklay, S. Fornasier, C. Feller, B. Davidsson, A. Gracia-Berná, B. Jost, R. Marschall, O. Poch, M.A. Barucci, J.-L. Bertaux, F. La Forgia, H.U. Keller, E. Kührt, S.C. Lowry, S. Mottola, G. Naletto, H. Sierks, C. Barbieri, P.L. Lamy, R. Rodrigo, D. Koschny, H. Rickman, J. Agarwal, M.F. A'Hearn, I. Bertini, S. Boudreault, G. Cremonese, V. Da Deppo, M. De Cecco, S. Debei, C. Güttler, M. Fulle, P.J. Gutierrez, S.F. Hviid, W.-H. Ip, L. Jorda, J. Knollenberg, G. Kovacs, J.-R. Kramm, E. Küppers, L. Lara, M. Lazzarin, J.L. Lopez Moreno, F. Marzari, H. Michalik, F. Preusker, F. Scholten, C. Tubiana, J.-B. Vincent, OSIRIS observations of meter-sized exposures of $\mathrm{H} 2 \mathrm{O}$ ice at the surface of 67P/Churyumov-Gerasimenko and interpretation using laboratory experiments. A\&A 583, 25 (2015a). doi:10.1051/0004-6361/201525977

A. Pommerol, B. Jost, O. Poch, M.R. El-Maarry, B. Vuitel, N. Thomas, The SCITEAS experiment: Optical characterizations of sublimating icy planetary analogues. Planet. Space Sci. 109, 106-122 (2015b). doi:10.1016/j.pss.2015.02.004

R.M. Pope, E.S. Fry, Absorption spectrum (380 -700 nm) of pure water. II. Integrating cavity measurements. Applied Optics 36, 8710-8723 (1997). doi:10.1364/AO.36.008710

F. Postberg, S. Kempf, J. Schmidt, N. Brilliantov, A. Beinsen, B. Abel, U. Buck, R. Srama, Sodium salts in E-ring ice grains from an ocean below the surface of Enceladus. Nature 459, 1098-1101 (2009). doi:10.1038/nature08046

P. Prem, N.A. Artemieva, D.B. Goldstein, P.L. Varghese, L.M. Trafton, Transport of water in a transient impact-generated lunar atmosphere. Icarus 255, 148-158 (2015).

doi:10.1016/j.icarus.2014.10.017

N. Riviere, R. Ceolato, L. Hespel, Polarimetric and angular light-scattering from dense media: Comparison of a vectorial radiative transfer model with analytical, stochastic and experimental approaches. J. Quant. Spec. Radiat. Transf. 131, 88-94 (2013).

doi:10.1016/j.jqsrt.2013.04.019

A.S. Rivkin, J.P. Emery, Detection of ice and organics on an asteroidal surface. Nature 464, 1322-1323 (2010). doi:10.1038/nature09028

V. Roloff, A. Pommerol, L. Gambicorti, A. Servonet, N. Thomas, M. Brändli, A. Casciello, G. Cremonese, V. Da Deppo, M. Erismann, I. Ficai Veltroni, M. Gerber, M. Gruber, P. Gubler, T. Hausner, M. Johnson, P. Lochmatter, E. Pel'o, B. Sodor, S. Szalai, G. Troznai, D. Vernani, T. Weigel, R. Ziethe, C. Zimmermann, On-Ground Performance and Calibration of the ExoMars Trace Gas Orbiter CaSSIS Imager. Space Sci. Rev. 212, 1871-1896 (2017). doi:10.1007/s11214-017-0404-2

T.L. Roush, J.B. Pollack, F.C. Witteborn, J.D. Bregman, J.P. Simpson, Ice and minerals on Callisto - A reassessment of the reflectance spectra. Icarus 86, 355-382 (1990). doi:10.1016/0019-1035(90)90225-X

P. Sabouroux, D. Ba, EPSIMU, a tool for dielectric properties measurement of porous media: Application in wet granular materials characterization, Pr. Electromagn. Res., 29, 191-207, 2011.

N.J. Sack, R.E. Johnson, J.W. Boring, R.A. Baragiola, The effect of magnetospheric ion bombardment on the reflectance of Europa's surface. Icarus 100, 534-540 (1992).

doi:10.1016/0019-1035(92)90116-O 
R.S. Saunders, F.P. Fanale, T.J. Parker, J.B. Stephens, S. Sutton, Properties of filamentary sublimation residues from dispersions of clay in ice. Icarus 66, 94-104 (1986). doi:10.1016/0019-1035(86)90009-6

H.M. Schmid, J.L. Beuzit, D. Mouillet, R. Waters, E. Buenzli, A. Boccaletti, K. Dohlen, M. Feldt, SPHERE Consortium, Polarimetry of extra-solar planets and circumstellar disks with ZIMPOL / SPHERE, in In the Spirit of Lyot 2010, 2010

B. Schmitt, D. Albert, P. Bollard, L. Bonal, P. Volcke, The Solid Spectroscopy Data Model (SSDM) and the GhoSST database, in EPSC-DPS Joint Meeting 2011, 2011, p. 1475

B. Schmitt, E. Quirico, F. Trotta, W. M. Grundy, Optical Properties of Ices from UV to Infrared, in Solar system ices, Dordrecht Kluwer Academic Publishers, Astrophysics and space science library (ASSL) Series vol no 227. ISBN0792349024 (1998). doi: 10.1007/978-94-011-5252-5_9

B. Schmitt, D. Albert, P. Bollard, L. Bonal, M. Gorbacheva, L. Mercier, S. Consortium Partners, SSHADE in H2020: Development of an European Database Infrastructure in Solid Spectroscopy. European Planetary Science Congress 10, 2015-628 (2015)

N. Schorghofer, Temperature response of Mars to Milankovitch cycles. Geophys. Res. Lett. 35, 18201 (2008). doi:10.1029/2008GL034954

N. Schorghofer, Migration calculations for water in the exosphere of the Moon: Duskdawn asymmetry, heterogeneous trapping, and $\mathrm{D} / \mathrm{H}$ fractionation. Geophys. Res. Lett. 41, 4888-4893 (2014). doi:10.1002/2014GL060820

F. Scipioni, F. Tosi, K. Stephan, G. Filacchione, M. Ciarniello, F. Capaccioni, P. Cerroni, Spectroscopic classification of icy satellites of Saturn I: Identification of terrain units on Dione. Icarus 226, 1331-1349 (2013). doi:10.1016/j.icarus.2013.08.008

F. Scipioni, F. Tosi, K. Stephan, G. Filacchione, M. Ciarniello, F. Capaccioni, P. Cerroni, Spectroscopic classification of icy satellites of Saturn II: Identification of terrain units on Rhea. Icarus 234, 1-16 (2014). doi:10.1016/j.icarus.2014.02.010

F. Scipioni, P. Schenk, F. Tosi, E. D’Aversa, R. Clark, J.-P. Combe, C.M.D. Ore, Deciphering sub-micron ice particles on Enceladus surface. Icarus 290, 183-200 (2017). doi:10.1016/j.icarus.2017.02.012

M.K. Shepard, P. Helfenstein, A test of the Hapke photometric model. Journal of Geophysical Research (Planets) 112, 03001 (2007). doi:10.1029/2005JE002625

Y.G. Shkuratov, P. Helfenstein, The Opposition Effect and the Quasi-fractal Structure of Regolith: I. Theory. Icarus 152, 96-116 (2001). doi:10.1006/icar.2001.6630

Y. Shkuratov, A. Ovcharenko, E. Zubko, O. Miloslavskaya, K. Muinonen, J. Piironen, R. Nelson, W. Smythe, V. Rosenbush, P. Helfenstein, The Opposition Effect and Negative Polarization of Structural Analogs for Planetary Regoliths. Icarus 159, 396-416 (2002). doi:10.1006/icar.2002.6923

Y. Shkuratov, V. Kaydash, V. Korokhin, Y. Velikodsky, D. Petrov, E. Zubko, D. Stankevich, G. Videen, A critical assessment of the Hapke photometric model. J. Quant. Spec. Radiat. Transf. 113, 2431-2456 (2012). doi:10.1016/j.jqsrt.2012.04.010 
Y. Shkuratov, V. Kaydash, V. Korokhin, Y. Velikodsky, D. Petrov, E. Zubko, D. Stankevich, G. Videen, Response to the comment by B. Hapke on "A critical assessment of the Hapke photometric model". J. Quant. Spec. Radiat. Transf. 116, 191-195 (2013). doi:10.1016/j.jqsrt.2012.11.004

A.P. Showman, R. Malhotra, The Galilean satellites. Science 296, 77-84 (1999)

M.A. Slade, B.J. Butler, D.O. Muhleman, Mercury radar imaging - Evidence for polar ice. Science 258, 635-640 (1992). doi:10.1126/science.258.5082.635

P.H. Smith, L.K. Tamppari, R.E. Arvidson, D. Bass, D. Blaney, W.V. Boynton, A. Carswell, D.C. Catling, B.C. Clark, T. Duck, E. DeJong, D. Fisher, W. Goetz, H.P. Gunnlaugsson, M.H. Hecht, V. Hipkin, J. Hoffman, S.F. Hviid, H.U. Keller, S.P. Kounaves, C.F. Lange, M.T. Lemmon, M.B. Madsen, W.J. Markiewicz, J. Marshall, C.P. McKay, M.T. Mellon, D.W. Ming, R.V. Morris, W.T. Pike, N. Renno, U. Staufer, C. Stoker, P. Taylor, J.A. Whiteway, A.P. Zent, H2o at the phoenix landing site. Science 325(5936), 58-61 (2009). doi:10.1126/science.1172339.

V. L. Snoeyink, D. Jenkins, Water Chemistry, Wiley (1980).

W.B. Sparks, J. Hough, T.A. Germer, F. Chen, S. DasSarma, P. DasSarma, F.T. Robb, N. Manset, L. Kolokolova, N. Reid, F.D. Macchetto, W. Martin, Detection of circular polarization in light scattered from photosynthetic microbes. Proceedings of the National Academy of Science 106, 7816-7821 (2009). doi:10.1073/pnas.0810215106

W.B. Sparks, B.E. Schmidt, M.A. McGrath, K.P. Hand, J.R. Spencer, M. Cracraft, S. E Deustua, Active Cryovolcanism on Europa? The Astrophys Journal Letters 839, 18 (2017). doi:10.3847/2041-8213/aa67f8

J.R. Spencer, T. Denk, Formation of lapetus' Extreme Albedo Dichotomy by Exogenically Triggered Thermal Ice Migration. Science 327, 432 (2010). doi:10.1126/science.1177132

J.R. Spencer, J.C. Pearl, M. Segura, F.M. Flasar, A. Mamoutkine, P. Romani, B.J. Buratti, A.R. Hendrix, L.J. Spilker, R.M.C. Lopes, Cassini Encounters Enceladus: Background and the Discovery of a South Polar Hot Spot. Science 311, 1401-1405 (2006). doi:10.1126/science.1121661

J.N. Spitale, C.C. Porco, Association of the jets of Enceladus with the warmest regions on its south-polar fractures. Nature 449, 695-697 (2007). doi:10.1038/nature06217

P.D. Spudis, D.B.J. Bussey, S.M. Baloga, B.J. Butler, D. Carl, L.M. Carter, M. Chakraborty, R.C. Elphic, J.J. Gillis-Davis, J.N. Goswami, E. Heggy, M. Hillyard, R. Jensen, R.L. Kirk, D. LaVallee, P. McKerracher, C.D. Neish, S. Nozette, S. Nylund, M. Palsetia, W. Patterson, M.S. Robinson, R.K. Raney, R.C. Schulze, H. Sequeira, J. Skura, T.W. Thompson, B.J. Thomson, E.A. Ustinov, H.L. Winters, Initial results for the north pole of the Moon from Mini-SAR, Chandrayaan-1 mission. Geophys. Res. Lett. 37, 06204 (2010). doi:10.1029/2009GL042259

J.K. Steckloff, B.C. Johnson, T. Bowling, H. Jay Melosh, D. Minton, C.M. Lisse, K. Battams, Dynamic sublimation pressure and the catastrophic breakup of Comet ISON. Icarus 258, 430-437 (2015). doi:10.1016/j.icarus.2015.06.032 
G. Steinbrügge, A. Stark, H. Hussmann, F. Sohl, J. Oberst, Measuring tidal deformations by laser altimetry. A performance model for the Ganymede Laser Altimeter. Planet. Space Sci. 117, 184-191 (2015). doi:10.1016/j.pss.2015.06.013

J.R. Stephens, B.A.S. Gustafson, Laboratory reflectance measurements of analogues to 'dirty' ice surfaces on atmosphereless solar system bodies. Icarus 94, 209-217 (1991). doi:10.1016/0019-1035(91)90151-I

S.A. Stern, F. Bagenal, K. Ennico, G.R. Gladstone, W.M. Grundy, W.B. McKinnon, J.M. Moore, C.B. Olkin, J.R. Spencer, H.A. Weaver, L.A. Young, T. Andert, J. Andrews, M. Banks, B. Bauer, J. Bauman, O.S. Barnouin, P. Bedini, K. Beisser, R.A. Beyer, S. Bhaskaran, R.P. Binzel, E. Birath, M. Bird, D.J. Bogan, A. Bowman, V.J. Bray, M. Brozovic, C. Bryan, M.R. Buckley, M.W. Buie, B.J. Buratti, S.S. Bushman, A. Calloway, B. Carcich, A.F. Cheng, S. Conard, C.A. Conrad, J.C. Cook, D.P. Cruikshank, O.S. Custodio, C.M. Dalle Ore, C. Deboy, Z.J.B. Dischner, P. Dumont, A.M. Earle, H.A. Elliott, J. Ercol, C.M. Ernst, T. Finley, S.H. Flanigan, G. Fountain, M.J. Freeze, T. Greathouse, J.L. Green, Y. Guo, M. Hahn, D.P. Hamilton, S.A. Hamilton, J. Hanley, A. Harch, H.M. Hart, C.B. Hersman, A. Hill, M.E. Hill, D.P. Hinson, M.E. Holdridge, M. Horanyi, A.D. Howard, C.J.A. Howett, C. Jackman, R.A. Jacobson, D.E. Jennings, J.A. Kammer, H.K. Kang, D.E. Kaufmann, P. Kollmann, S.M. Krimigis, D. Kusnierkiewicz, T.R. Lauer, J.E. Lee, K.L. Lindstrom, I.R. Linscott, C.M. Lisse, A.W. Lunsford, V.A. Mallder, N. Martin, D.J. McComas, R.L. McNutt, D. Mehoke, T. Mehoke, E.D. Melin, M. Mutchler, D. Nelson, F. Nimmo, J.I. Nunez, A. Ocampo, W.M. Owen, M. Paetzold, B. Page, A.H. Parker, J.W. Parker, F. Pelletier, J. Peterson, N. Pinkine, M. Piquette, S.B. Porter, S. Protopapa, J. Redfern, H.J. Reitsema, D.C. Reuter, J.H. Roberts, S.J. Robbins, G. Rogers, D. Rose, K. Runyon, K.D. Retherford, M.G. Ryschkewitsch, P. Schenk, E. Schindhelm, B. Sepan, M.R. Showalter, K.N. Singer, M. Soluri, D. Stanbridge, A.J. Ste $\Leftrightarrow$, D.F. Strobel, T. Stryk, M.E. Summers, J.R. Szalay, M. Tapley, A. Taylor, H. Taylor, H.B. Throop, C.C.C. Tsang, G.L. Tyler, O.M. Umurhan, A.J. Verbiscer, M.H. Versteeg, M. Vincent, R. Webbert, S. Weidner, G.E. Weigle, O.L. White, K. Whittenburg, B.G. Williams, K. Williams, S. Williams, W.W. Woods, A.M. Zangari, E. Zirnstein, The Pluto system: Initial results from its exploration by New Horizons. Science 350, 1815 (2015). doi:10.1126/science.aad1815

C.R. Stoker, A. Zent, D.C. Catling, S. Douglas, J.R. Marshall, D. Archer, B. Clark, S.P. Kounaves, M.T. Lemmon, R. Quinn, N. Renno, P.H. Smith, S.M.M. Young, Habitability of the Phoenix landing site. Journal of Geophysical Research (Planets) 115, 00-20 (2010). doi:10.1029/2009JE003421

A.D. Storrs, F.P. Fanale, R.S. Saunders, J.B. Stephens, The formation of filamentary sublimate residues (FSR) from mineral grains. Icarus 76, 493-512 (1988).

doi:10.1016/0019-1035(88)90017-6

G. Strazzulla, G.A. Baratta, G. Leto, G. Foti, lon-beam-induced amorphization of crystalline water ice. EPL (Europhysics Letters) 18, 517 (1992). doi:10.1209/0295$5075 / 18 / 6 / 008$

G. Strazzulla, M. Garozzo, D. Fulvio, Z. Kanuchova, M.A. Palumbo, Cosmic ion induced chemistry in ices. Rendiconti Lincei 22(2), 145-152 (2011). doi:10.1007/s12210-0110121-9. http://dx.doi.org/10.1007/s12210-011-0121-9

C.M. Stuurman, G.R. Osinski, J.W. Holt, J.S. Levy, T.C. Brothers, M. Kerrigan, B.A. Campbell, SHARAD detection and characterization of subsurface water ice deposits in Utopia Planitia, Mars. Geophys. Res. Lett., 43, 9484-9491 (2016).

doi:10.1002/2016GL070138 
J.M. Sunshine, M.F. A'Hearn, O. Groussin, J.-Y. Li, M.J.S. Belton, W.A. Delamere, J. Kissel, K.P. Klaasen, L.A. McFadden, K.J. Meech, H.J. Melosh, P.H. Schultz, P.C. Thomas, J. Veverka, D.K. Yeomans, I.C. Busko, M. Desnoyer, T.L. Farnham, L.M. Feaga, D.L. Hampton, D.J. Lindler, C.M. Lisse, D.D. Wellnitz, Exposed Water Ice Deposits on the Surface of Comet 9P/Tempel 1. Science 311, 1453-1455 (2006). doi:10.1126/science.1123632

J.M. Sunshine, L.M. Feaga, O. Groussin, S. Protopapa, M.F. A'Hearn, T.L. Farnham, S. Besse, DIXI Team, The Distribution of Water Ice on Comet 103P/Hartley 2. LPI Contributions 1667, 6438 (2012)

C. Szopa, G. Cernogora, L. Boufendi, J.J. Correia, P. Coll, PAMPRE: A dusty plasma experiment for Titan's tholins production and study. Planet. Space Sci. 54, 394-404 (2006). doi:10.1016/j.pss.2005.12.012

D.J. Tholen, Asteroid taxonomy from cluster analysis of photometry, PhD thesis, University of Arizona, Tucson, 1984

N. Thomas, T. Spohn, J.-P. Barriot, W. Benz, G. Beutler, U. Christensen, V. Dehant, C. Fallnich, D. Giardini, O. Groussin, K. Gunderson, E. Hauber, M. Hilchenbach, L. less, P. Lamy, L.-M. Lara, P. Lognonné, J.J. Lopez-Moreno, H. Michaelis, J. Oberst, D.

Resendes, J.-L. Reynaud, R. Rodrigo, S. Sasaki, K. Seiferlin, M. Wieczorek, J. Whitby, The BepiColombo Laser Altimeter (BELA): Concept and baseline design. Planet. Space Sci. 55, 1398-1413 (2007). doi:10.1016/j.pss.2007.03.003

N. Thomas, B. Davidsson, M.R. El-Maarry, S. Fornasier, L. Giacomini, A.G. GraciaBerná, S.F. Hviid, W.-H. Ip, L. Jorda, H.U. Keller, J. Knollenberg, E. Kührt, F. La Forgia, I.L. Lai, Y. Liao, R. Marschall, M. Massironi, S. Mottola, M. Pajola, O. Poch, A. Pommerol, F. Preusker, F. Scholten, C.C. Su, J.S. Wu, J.-B. Vincent, H. Sierks, C. Barbieri, P.L. Lamy, R. Rodrigo, D. Koschny, H. Rickman, M.F. A'Hearn, M.A. Barucci, J.-L. Bertaux, I. Bertini, G. Cremonese, V. Da Deppo, S. Debei, M. de Cecco, M. Fulle, O. Groussin, P.J. Gutierrez, J.-R. Kramm, M. Küppers, L.M. Lara, M. Lazzarin, J.J. Lopez Moreno, F. Marzari, H. Michalik, G. Naletto, J. Agarwal, C. Güttler, N. Oklay, C. Tubiana, Redistribution of particles across the nucleus of comet $67 \mathrm{P} /$ Churyumov-Gerasimenko. A\&A 583, 17 (2015). doi:10.1051/0004-6361/201526049

N. Thomas, G. Cremonese, R. Ziethe, M. Gerber, M. Brändli, G. Bruno, M. Erismann, L. Gambicorti, T. Gerber, K. Ghose, M. Gruber, P. Gubler, H. Mischler, J. Jost, D. Piazza, A. Pommerol, M. Rieder, V. Roloff, A. Servonet, W. Trottmann, T. Uthaicharoenpong, C. Zimmermann, D. Vernani, M. Johnson, E. Pel’o, T. Weigel, J. Viertl, N. De Roux, P. Lochmatter, G. Sutter, A. Casciello, T. Hausner, I. Ficai Veltroni, V. Da Deppo, P. Orleanski, W. Nowosielski, T. Zawistowski, S. Szalai, B. Sodor, S. Tulyakov, G. Troznai, M. Banaskiewicz, J.C. Bridges, S. Byrne, S. Debei, M.R. El-Maarry, E. Hauber, C.J. Hansen, A. Ivanov, L. Keszthelyi, R. Kirk, R. Kuzmin, N. Mangold, L. Marinangeli, W.J. Markiewicz, M. Massironi, A.S. McEwen, C. Okubo, L.L. Tornabene, P. Wajer, J.J. Wray, The Colour and Stereo Surface Imaging System (CaSSIS) for the ExoMars Trace Gas Orbiter. Space Sci. Rev. 212, 1897-1944 (2017). doi:10.1007/s11214-017-0421-1

P.C. Thomas, R. Tajeddine, M.S. Tiscareno, J.A. Burns, J. Joseph, T.J. Loredo, P. Helfenstein, C. Porco, Enceladus's measured physical libration requires a global subsurface ocean. Icarus 264, 37-47 (2016). doi:10.1016/j.icarus.2015.08.037 
T.W. Thompson, E.A. Ustinov, E. Heggy, Modeling radar scattering from icy lunar regoliths at $13 \mathrm{~cm}$ and $4 \mathrm{~cm}$ wavelengths. Journal of Geophysical Research (Planets) 116, 01006 (2011). doi:10.1029/2009JE003368

L.L. Tornabene, F.P. Seelos, A. Pommerol, K.T. Hansen, N. Segal, N. Thomas, G. Cremonese, A.S. McEwen, S. Sutton, M. Chojnacki, Analysis of Colour and Stereo Surface Imaging System (CaSSIS) Colour Capabilities and Simulated Images Generated from MRO Datasets, in Lunar and Planetary Science Conference. Lunar and Planetary Science Conference, vol. 47, 2016, p. 2695

B.J. Travis, G. Schubert, Keeping Enceladus warm. Icarus 250, 32-42 (2015). doi:10.1016/j.icarus.2014.11.017

S.A. Twomey, C.F. Bohren, J.L. Mergenthaler, Reflectance and albedo differences between wet and dry surfaces. Applied Optics 25, 431-437 (1986).

doi:10.1364/AO.25.000431

F.T. Ulaby, D.G. Long, Microwave Radar and Radiometric Remote Sensing (Univ. Michigan Press, 2014)

A. Virkki, K. Muinonen, Radar scattering by planetary surfaces modeled with laboratorycharacterized particles. Icarus 269, 38-49 (2016). doi:10.1016/j.icarus.2016.01.011

A.J. Verbiscer, J. Veverka, Scattering properties of natural snow and frost - Comparison with icy satellite photometry. Icarus 88, 418-428 (1990). doi:10.1016/00191035(90)90092-N

A. Vorburger, P. Wurz, H. Lammer, S. Barabash, O. Mousis, Monte-Carlo simulation of Callisto's exosphere. Icarus 262, 14-29 (2015). doi:10.1016/j.icarus.2015.07.035

W. Wagner, A. Pruß, The IAPWS Formulation 1995 for the Thermodynamic Properties of Ordinary Water Substance for General and Scientific Use. Journal of Physical and Chemical Reference Data 31, 387-535 (2002). doi:10.1063/1.1461829

S.D. Wall, S.L. McConnell, C.E. Leff, R.S. Austin, K.K. Beratan, M.J. Rokey, User guide to the Magellan synthetic aperture radar images 1995

F. Westall, A. Brack, The Importance of Water for Life. Space Science Reviews 214(50) (2018)

O.L. White, O.M. Umurhan, J.M. Moore, A.D. Howard, Modeling of ice pinnacle formation on Callisto. Journal of Geophysical Research (Planets) 121, 21-45 (2016).

doi:10.1002/2015JE004846

O. Wilkman, K. Muinonen, J. Peltoniemi, Photometry of dark atmosphereless planetary bodies: an efficient numerical model. Planet. Space Sci. 118, 250-255 (2015).

doi:10.1016/j.pss.2015.06.004

X. Wu, W. Dai, X. Shan, W. Wang, L. Tang, Visual and theoretical analyses of the early stage of frost formation on cold surfaces. Journal of Enhanced Heat Transfer 14, 1-12 (2007)

M.T. Zuber, J.W. Head, D.E. Smith, G.A. Neumann, E. Mazarico, M.H. Torrence, O. Aharonson, A.R. Tye, C.I. Fassett, M.A. Rosenburg, H.J. Melosh, Constraints on the 
volatile distribution within Shackleton crater at the lunar south pole. Nature 486, 378-381 (2012). doi:10.1038/nature11216 


\section{Acknowledgements}

Most of this work was financed by the Swiss National Science Foundation, in particular under the NCCR PlanetS. Significant contributions to the funding of this project by the University of Bern and especially its Centre for Space and Habitability are also acknowledged. O. P. acknowledges a postdoctoral fellowship from CNES.

We thank two anonymous reviewers for their careful reading of the manuscript and their useful and constructive reviews. We are also grateful to the organizers of the Ices in the Solar System conference in Madrid for the organisation of a stimulating and fruitful meeting. 


\section{Table captions}

Table 1: A summary of different remote sensing techniques based on the interaction between the regolith and electromagnetic field at different wavelengths over the entire spectrum.

Table 2: Parameters of the Hapke model. See Figure 1 for illustrations of the definition and role of the parameter.

Table 3: Instruments and facilities developed and/or used in the Ice Laboratory and by collaborators.

Table 4: Parameters and properties of the different ice production setups developed and their products.

Table 5: Summary of ice-dust mixtures whose spectro-photometric properties were measured in the LOSSy and published

Table 6: Summary of samples properties and measurements of the real part of the permittivity, $\varepsilon$ ', in the $0.05-2 \mathrm{GHz}$ range (from Brouet et al., 2015, 2016a, 2016b).

Table 7: Objectives and technical solutions for the new version of the SCITEAS simulation chamber (SCITEAS-2) 


\section{Figure captions}

Figure 1: Illustration of the Hapke model with identification of the key parameters that control the calculated reflectance of the surface. See Table 2 for definitions of the parameters.

Figure 2: Schematics of the Setup for the Preparation of Icy Planetary Analogues - A (SPIPA-A). This setup uses an ultrasonic nebuliser to produce liquid droplets a few micrometres in size. The droplets are made of water in which various molecules can be dissolved. The droplets freeze in cold air and sediment into a metallic bowl kept at low temperature by liquid nitrogen. The icy particles produced are spherical and have a mean diameter of about 5 micrometres.

Figure 3: Schematics of the Setup for the Preparation of Icy Planetary Analogues - B (SPIPA-B). Droplets of a solution of dissolved particles or suspension of solid particles in water are spread into liquid nitrogen through the sonotrode of a ultrasonic nebuliser. The icy particles produced are spherical and have a mean diameter of about 70 micrometres.

Figure 4: Schematics of the Setup for the Preparation of Icy Planetary Analogues - C (SPIPA-C). Droplets of a solution of dissolved particles or suspension of solid particles in water are spread into liquid nitrogen using a nebuliser nozzle and compressed air. The icy particles produced are spherical and have very broad size distribution with particles ranging between a few micrometres and hundreds of micrometes.

Figure 5: Production of an inter-mixture of ice and dust by mechanical mixing on a vortex mixer. The ice and dust are kept at low temperature in an aluminium bottle that is regularly plunged into liquid nitrogen.

Figure 6: CAD drawings of the sample holder built for the deposition of frost on substrates made of sand and dust. For the production of frost, the blue cylinder is filled with liquid nitrogen to cool down the violet piece made of copper. The orange part is covered by a thick piece of insulating foam to ensure that the coldest point is the surface of the dust layer, on which frost condenses.

Figure 7: (left) Optical microscope picture of a sample of water frost growing on a substrate of JSC Mars-1. Note that the orange grains of the substrate made of the JSC Mars- 
1 soil analogue are still visible through the needles of the frost layer. (right) 3D-rendering made from an Optical Coherence Tomography image (See Section 3.2.2) of the same sample. The volume rendered is $2 \times 2 \times 2$ millimetres in size.

Figure 8: Optical microscope pictures of three different samples of frozen soils. Each image is 6 millimetres wide. Sample A was obtained by simply leaving a wet sample of the JSC Mars-1 soil simulant in a freezer at 243K overnight. The grains of JSC Mars-1 are surrounded by a matrix of ice in the bulk of the sample but their upper parts at the surface remain free of ice. To produce samples B and C, we sprayed various amounts of liquid water over the surface of the cold sample A to form a thin conformal layer of ice that follows the topography of the grains (B) or a much thicker layer of ice that hides the topography of the underlying grains $(\mathrm{C})$.

Figure 9: Comparison of optical microscope images at the same scale of an inter-mixture (left) and an intra-mixture (right) of SPIPA-B water ice and tholins. Each image is 2.5 millimetres wide and shows the spherical particles of SPIPA-B with mean diametres of 70 micrometres. In the case of the inter-mixture on the left, yellowish agglomerates of tholins coat parts of the surfaces of some of the large particles of ice. In the case of the intramixture on the right, the tholins are enclosed inside the particles of ice. The reflectance spectra of these samples are presented in Poch et al. 2016b.

Figure 10: Scanning Electron Microscope (SEM) images of inter-mixtures of SPIPA-A water ice particles and basaltic particles of the JSC1-AF lunar dust analogue. The two typers of particles are easily distinguishable by their shapes: the ice particles are roundish and largely affected by sintering whereas the dust particles are angular and overall larger.

Figure 11: Example of a 3D rendering of the surface of a sample generated from Optical Coherence Tomography (OCT) data. In this case, the sample was composed of the smectite SWy-2, which was initially mixed (1 wt. \%) with SPIPA-B water ice. Even after complete desiccation of the upper layers of the sample, the structure of the spheres of SPIPA-B ice can still be clearly seen at the surface. The rendered volume is $6 \times 3 \times 1 \mathrm{~mm}$ in size $(\mathrm{L}, \mathrm{w}, \mathrm{h})$

Figure 12: Optical (a,b,c,f) and Scanning Electron Microscope (d,e) images of sublimation residues of different composition. Left (a,d): tholins; Center (b,e): smectite SWy-2; Right $(c, f)$ : olivine. The SEM images are modified from Figure 4a,d in Poch et al. 2016b, Copyright (2016), with permission from Elsevier. 
Figure 13: Interpretative scenarios for the sublimation of (left) intra-mixture and (right) inter-mixture of SPIPA-B ice and dust. Reprinted from Figure 8 in Poch et al. 2016b, Copyright (2016), with permission from Elsevier.

Figure 14: Reflectance spectra measured on four different sublimation residues (red curves) compared to the reflection spectra of the pure refractory material prior to mixing (grey curves) and the initial icy mixture (black curve).

Figure 15: Schematic representation of comet sublimation experiment \#3 from Jost et al. 2017a. The original sample is made of $98 \%$ (wt.) of water ice, $1.8 \%$ of activated charcoal and $0.2 \%$ of titan tholins. The material is prepared once as inter-mixture (left) and one as intra-mixture (right). Subfigures (a)-(d) show the samples at various points during the sublimation and insolation process. (e) represents the VIS-NIR spectral shortly after start and before the end of the experiment. (f) shows the spectral ratio between two regions of interest of the intra-mixture inside and outside the illuminated area. The spectral ratio clearly shows the higher water ice content inside the illuminated area where resurfacing took place and thickness of the residue layer is reduced. (g) and (h) show the reflectance phase curves before and after some hours of sublimation at $\mathrm{i}=60^{\circ}$ in absolute units of reflectance factor and normalized at $\backslash a l p h a=5^{\circ}$. The decreased fraction of forward scattered light indicates an increase macroscopic roughness of the samples. For more details see discussion on Jost et al. 2017a.

Figure 16: Measured phase curves (a-d) and reflectance spectra (e-f) of binary mixtures of the JSC1-AF simulant for fine lunar dust with SPIPA-A (top) and SPIPA-B (bottom) ices in variable concentrations.

Figure 17: Several analogues of Martian soils and their corresponding reflectance spectra. a) JSCM-1 and SPIPA-A intimate mixtures. The concentration of water ice is indicated next to each sample b) Reflectance spectra of JSCM-1 and SPIPA-A intimate mixtures. Each color corresponds to a water-ice concentration, as indicated in the legend c) Frost cold trapped onto four size-distributions of JSCM-1 d) Reflectance spectra of three frost depositions of frost onto the fine size-distribution of JSCM-1 e) Frozen soils of JSCM-1 after $25 \mathrm{~h}$ of sublimation. On the bottom left the substrate has been exposed. The orange rectangle indicates the region of interest used to measure the reflectance spectra $f$ ) Reflectance spectra of the exposed sample of JSCM-1 during 25 hours g) RED-PAN-BLU CaSSIS composite of the sample shown in e) h) NIR-RED-PAN CaSSIS composite of the sample shown in e). 
Figure 18: Ternary diagram of the real part of the permittivity as a function of the volume fraction of silicate-rich refractory material (JSC-1A lunar simulant), vacuum, and water ice, valid for 0.05-2 GHz and at a temperature of $243 \mathrm{~K}$ using equation (2) in Brouet et al., $2016 b$.

Figure 19: Comparison of the effects of macroscopic roughness on the phase curves of a surface modelled with the Hapke (1993) model (lines) and the ray-tracing model of GraciaBerná et al. (2016) using fractal meshes (+ signs) for average slope angles varying between $0^{\circ}$ (smooth) and $50^{\circ}$ by steps of $10^{\circ}$. All simulations and calculations were performed for an incidence angle: $\mathrm{i}=30^{\circ}$. Both models use as inputs the reflectance data measured on a smooth surface made of a fine powder of the Allende meteorite.

Figure 20: Optical microscope picture and interpretative schematics of a sample produced by spraying a solution of $7.4 \mathrm{wt} . \%$ of $\mathrm{MgSO}_{4}$ prepared at $20^{\circ} \mathrm{C}$ with the SPIPA-B setup. As spheres of water ice cristallize with diameters of few tens to hundreds of $\mu \mathrm{m}$, smaller (a few $\mu \mathrm{m}$ to tens of $\mu \mathrm{m}$ ) and angular crystals of $\mathrm{MgSO}_{4}, \mathrm{X}-\mathrm{H}_{2} \mathrm{O}$ (X is unknown, but could be as high as 11; McCarthy et al., 2011) cristallize around the ice spheres. 


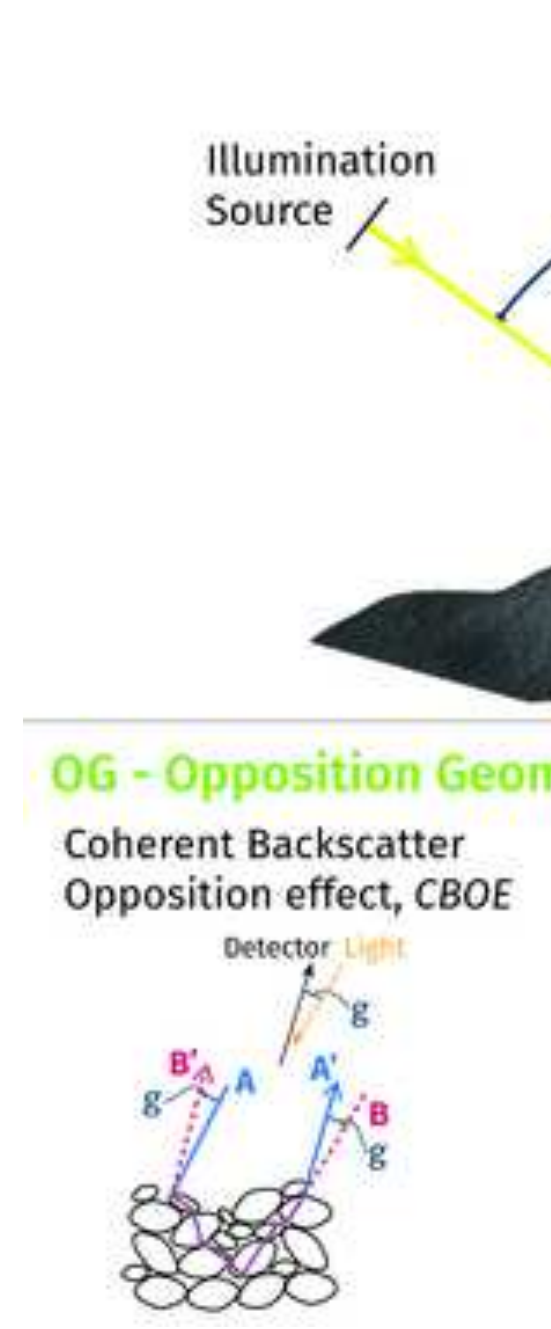

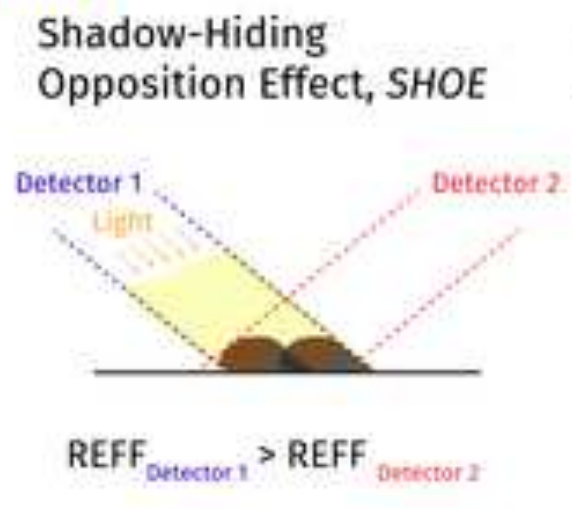

\section{Z1 - Intimate mixtures.}

Mixing formulas.

$\leftarrow$ Relative concentration.

$\llcorner$ Sphere-equivalent diameter, $d$.

Porosity, $p$

$\mathrm{H}$-functions, $\mathrm{H}$

Mean slope angle, $\dot{\theta}[0]$

$\theta_{s}$

21
Isotropic multi scattering approx., IMSA

\section{2 - Single particle}

Angular width parameter of CBOE and SHOE, $h_{c}, h_{s}$

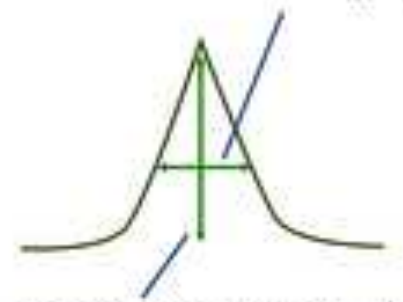

Amplitude of $\mathrm{CBOE}, \mathrm{B}_{\mathrm{co}}$ Amplitude of SHOE, $B_{50}$

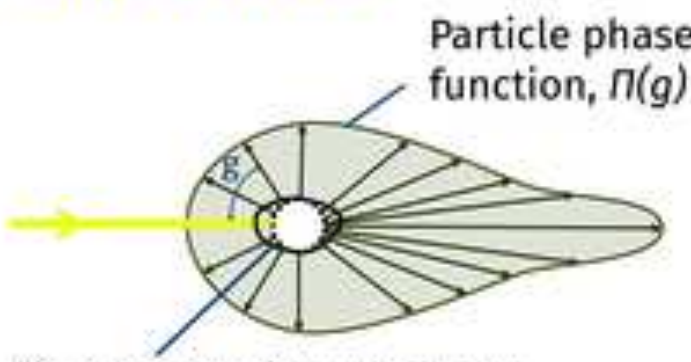

Single scattering albedo, $\omega$ 。 $\leftarrow$ Sphere-equivalent diameter, $d$

- Refraction index, $n, k$ 


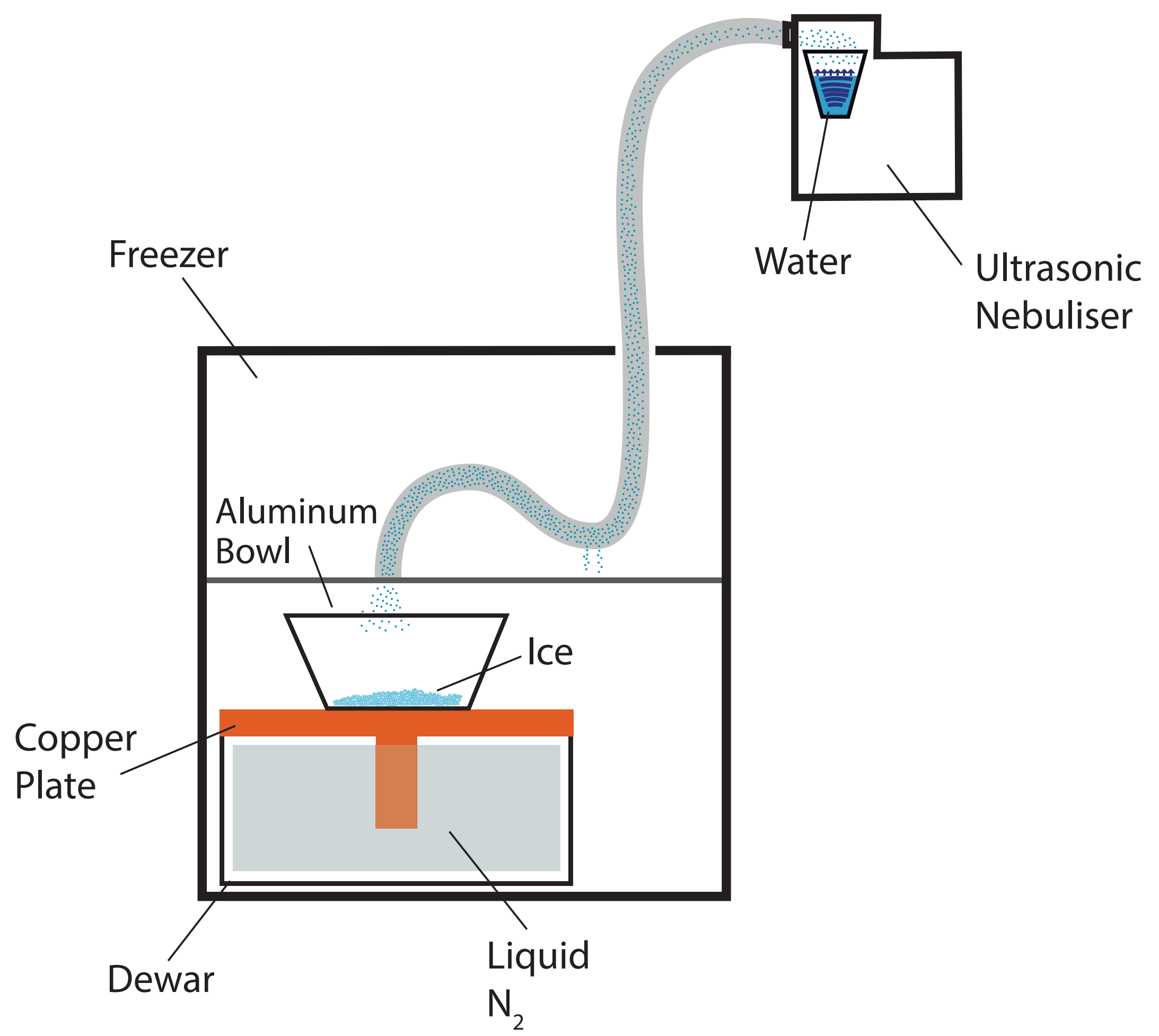


Click here to access/download;figure;Figure3.eps $\underline{\underline{ \pm}}$

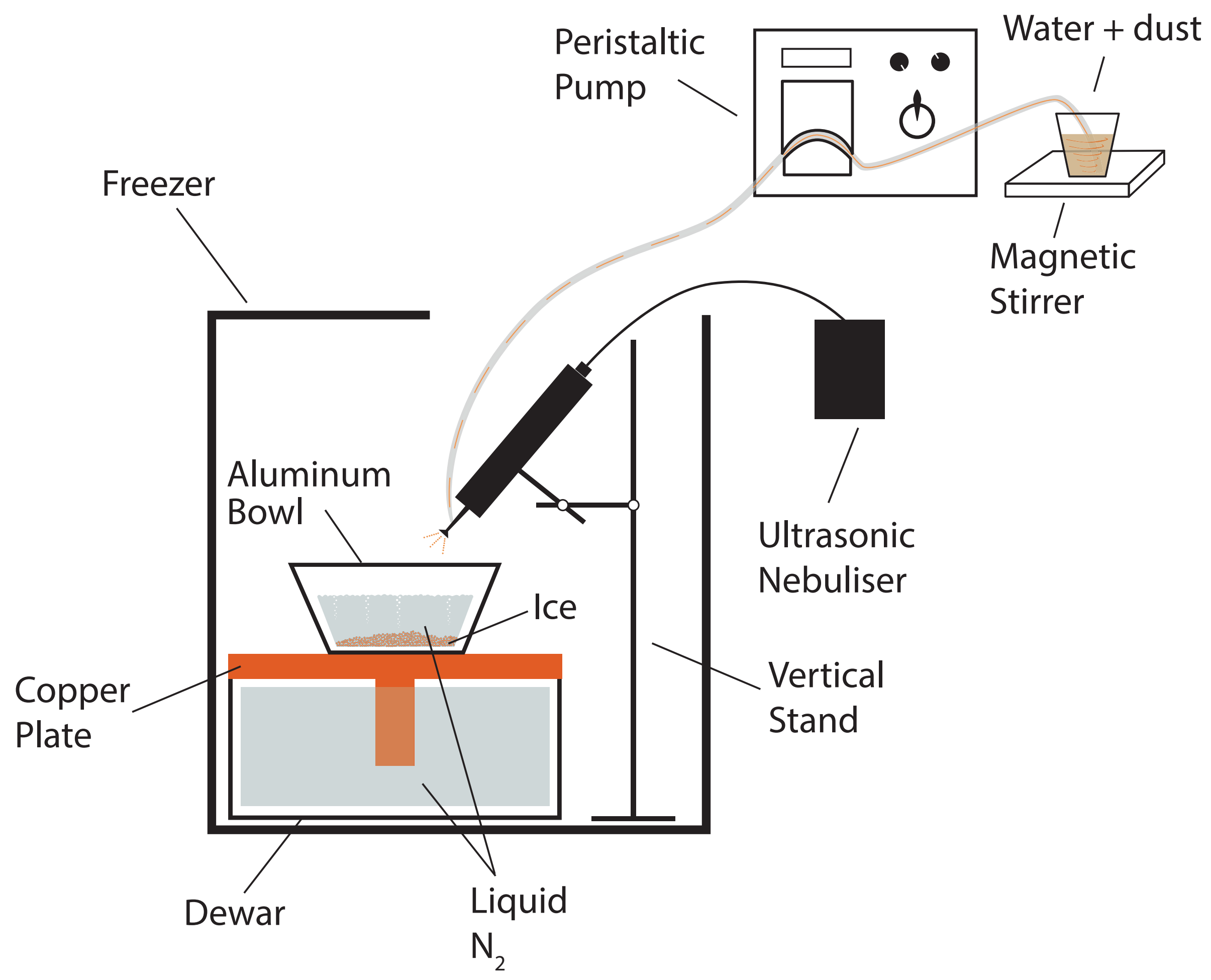




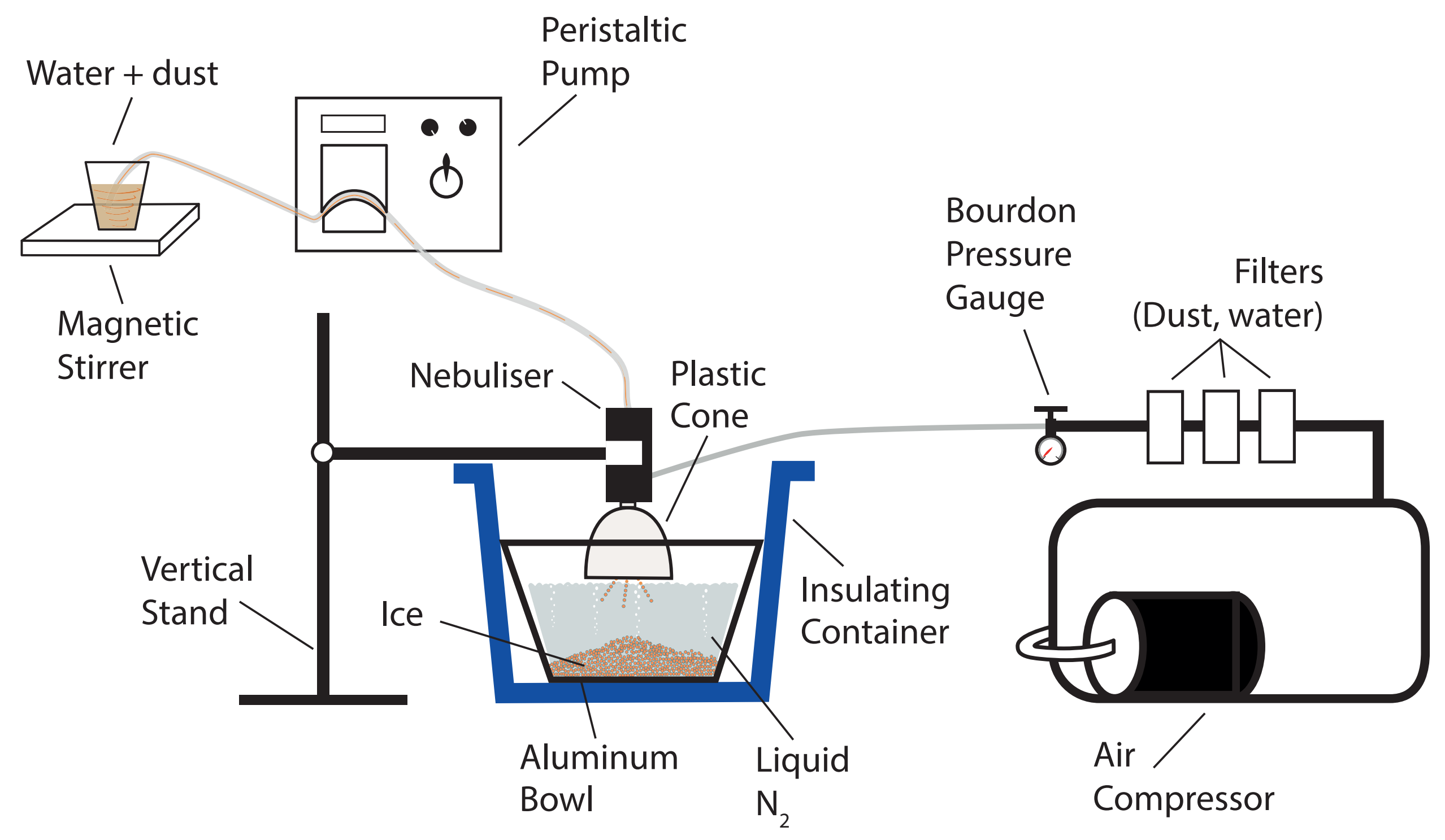




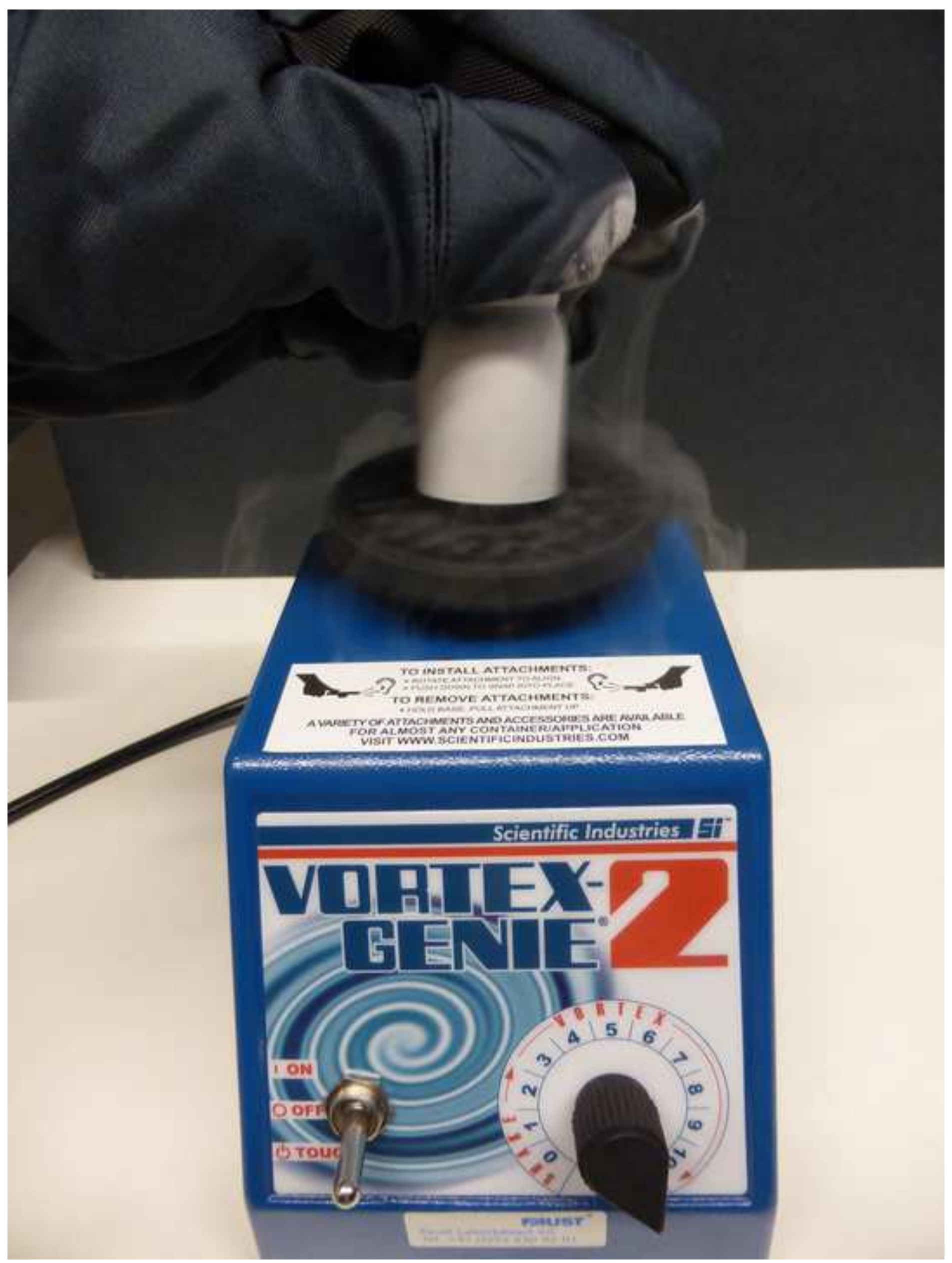




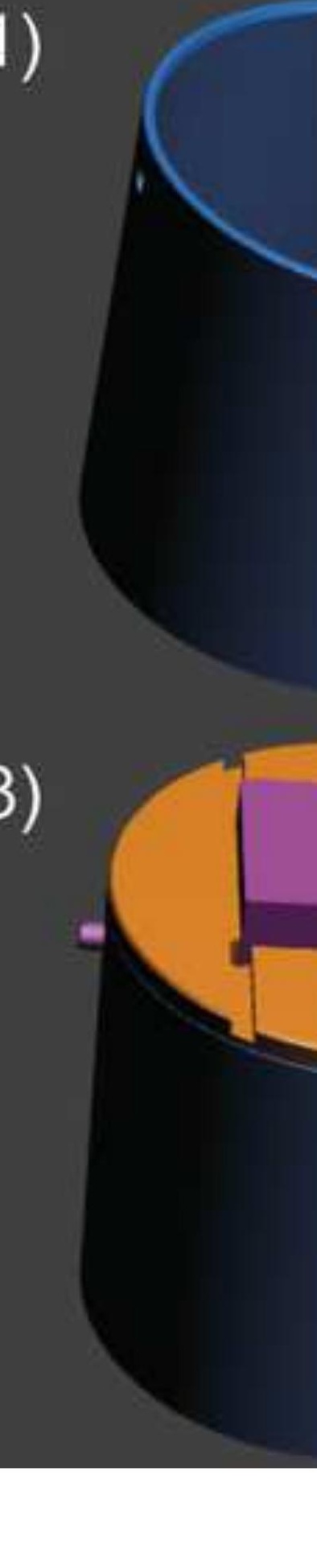

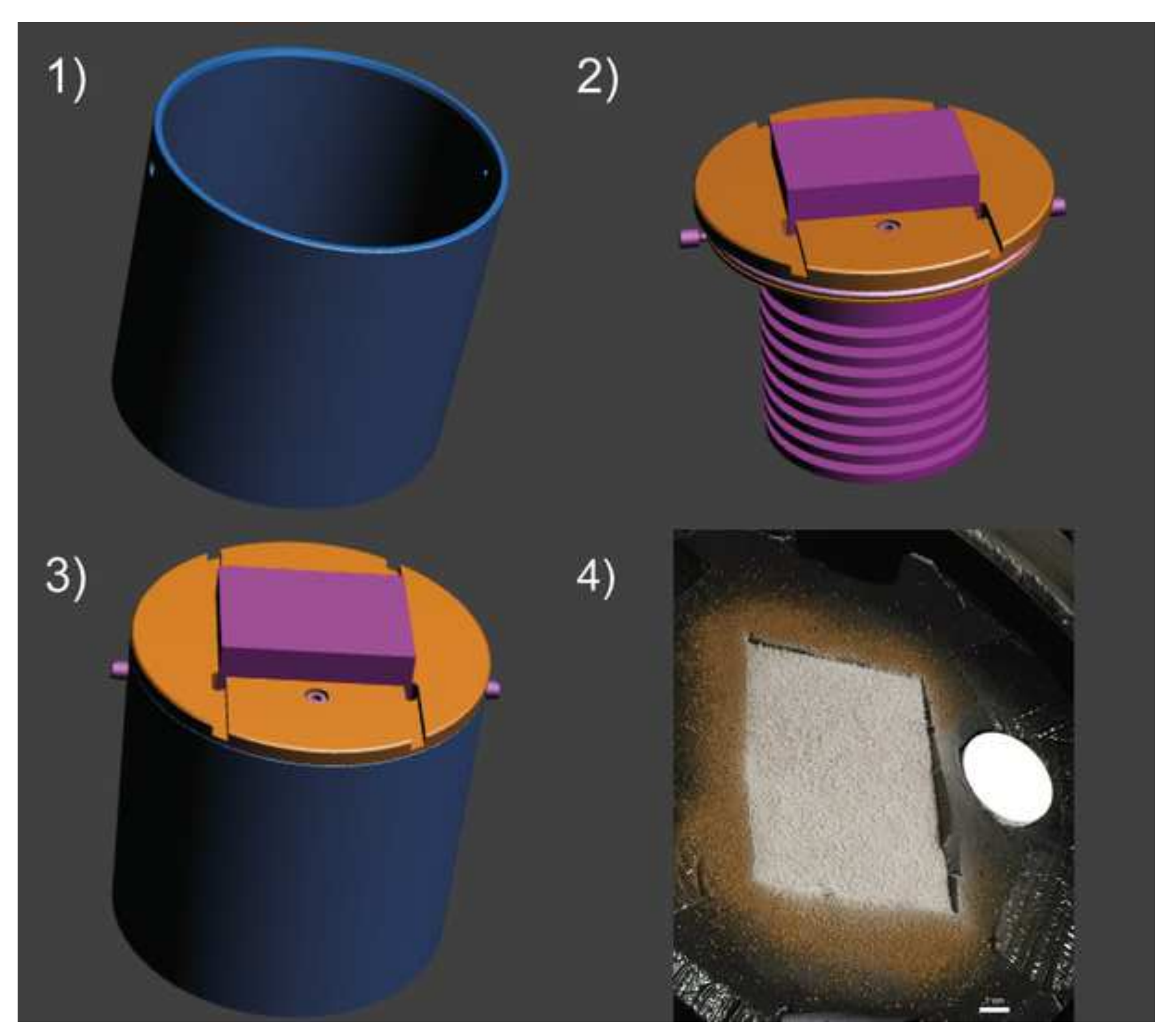

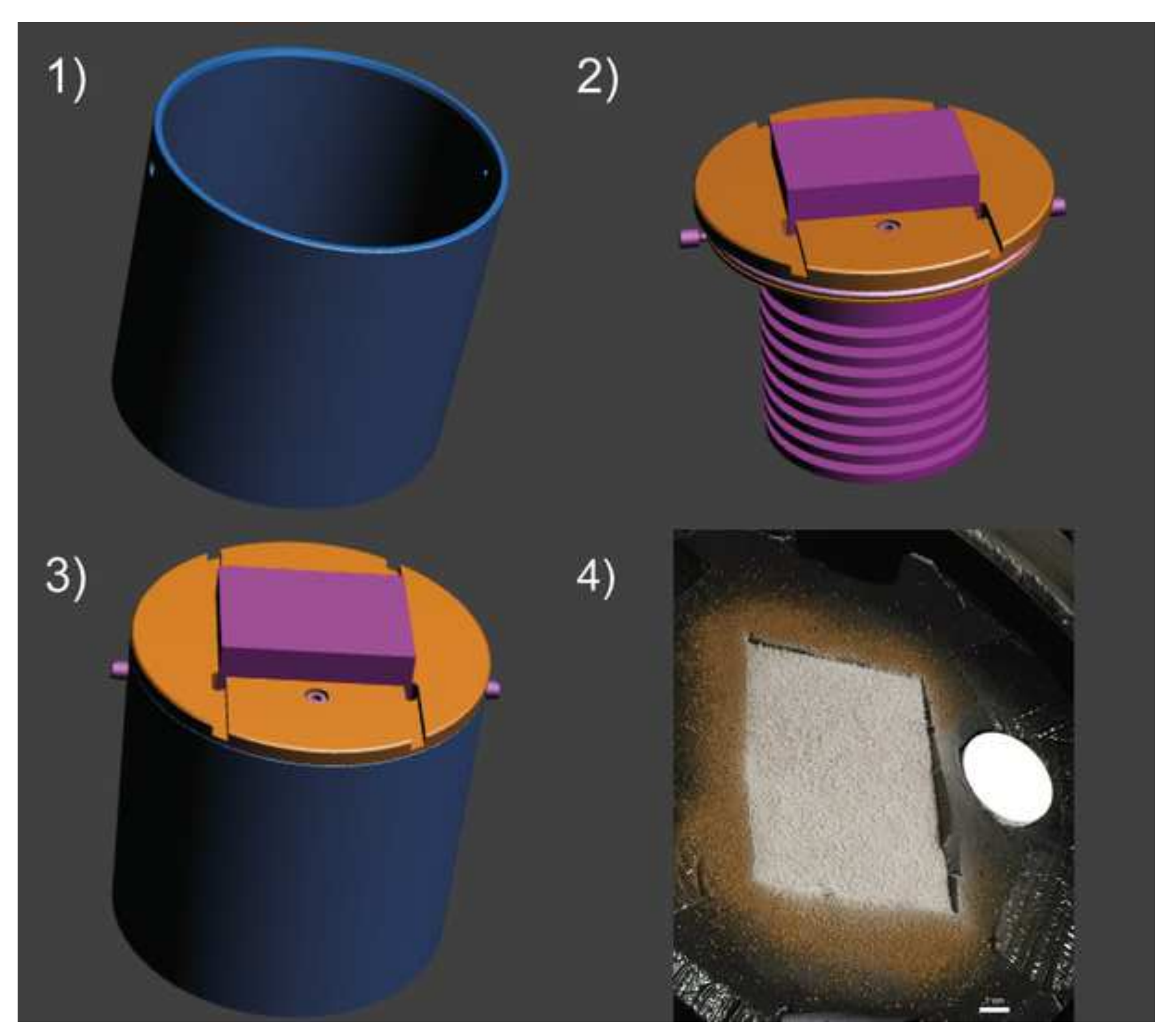

2)

4)

\section{2)}

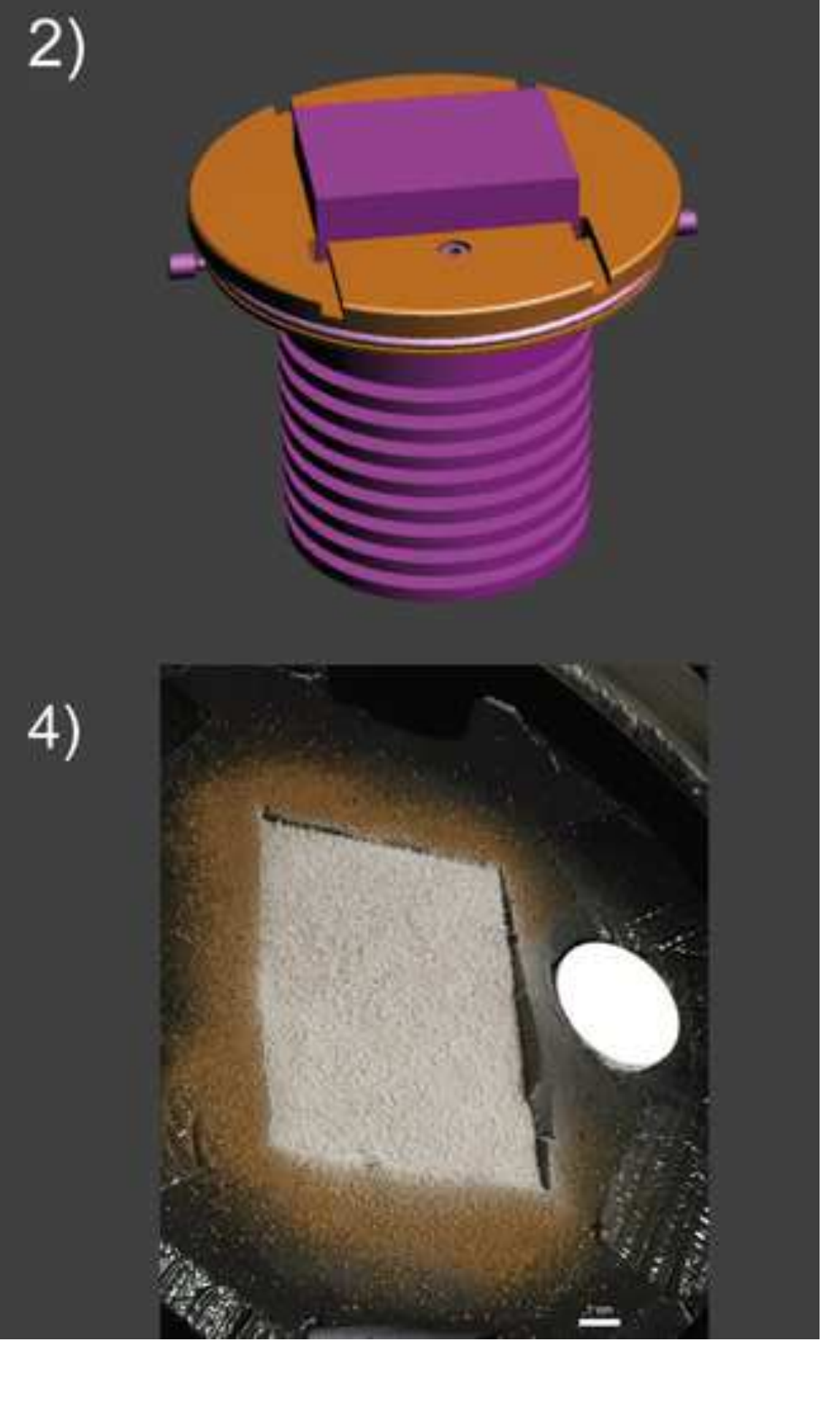

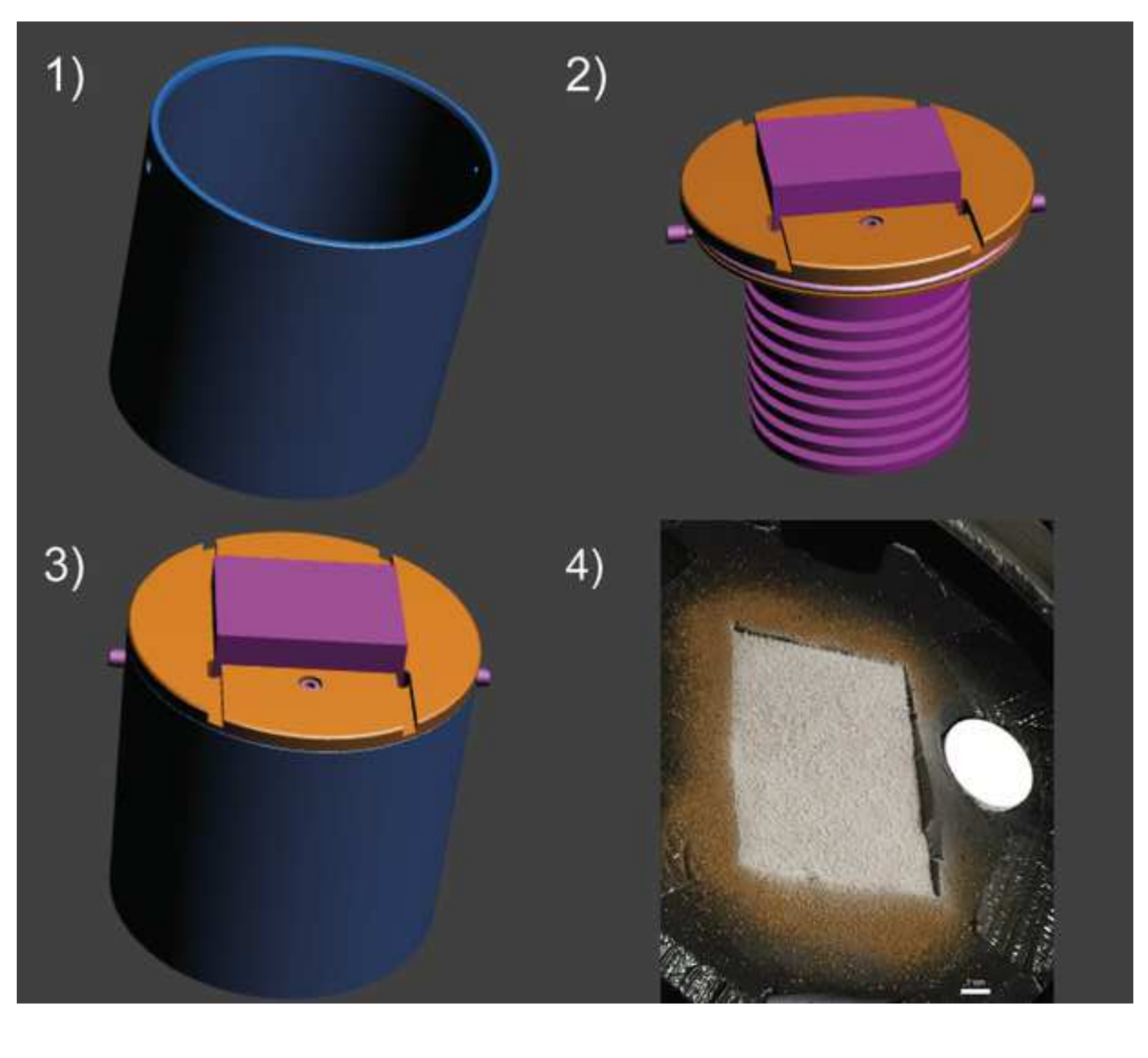

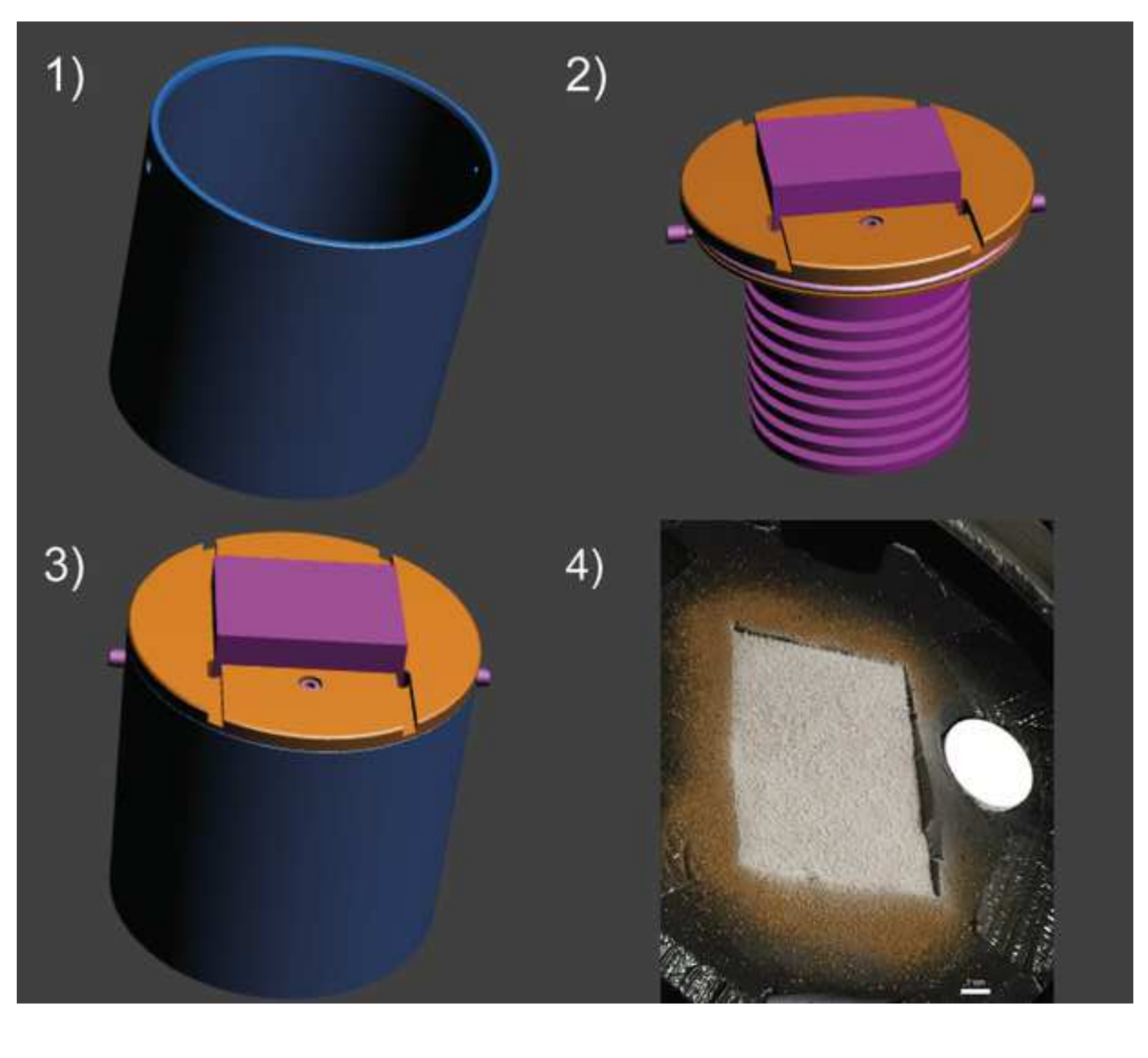
3)

\section{1)}

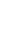

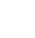
t
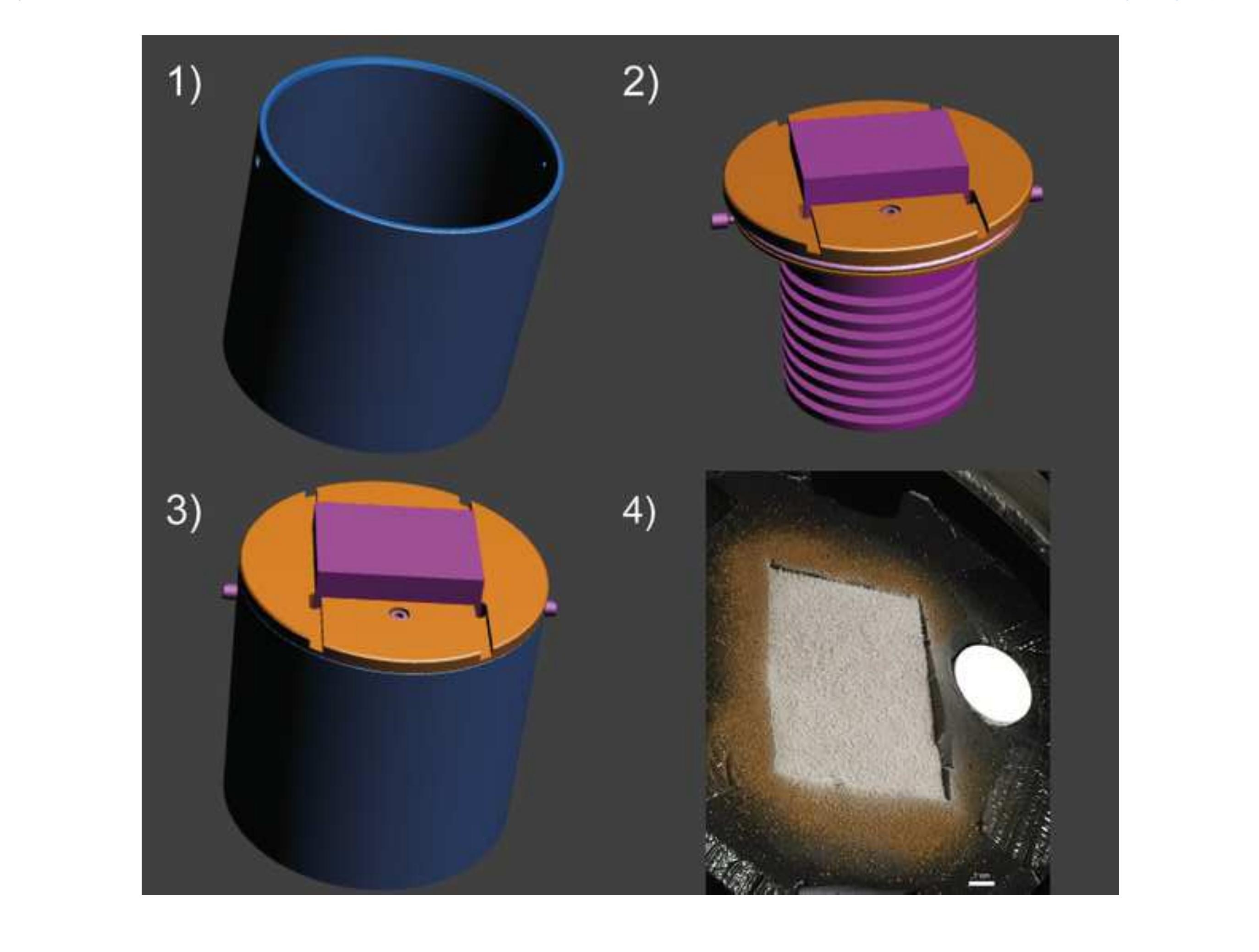

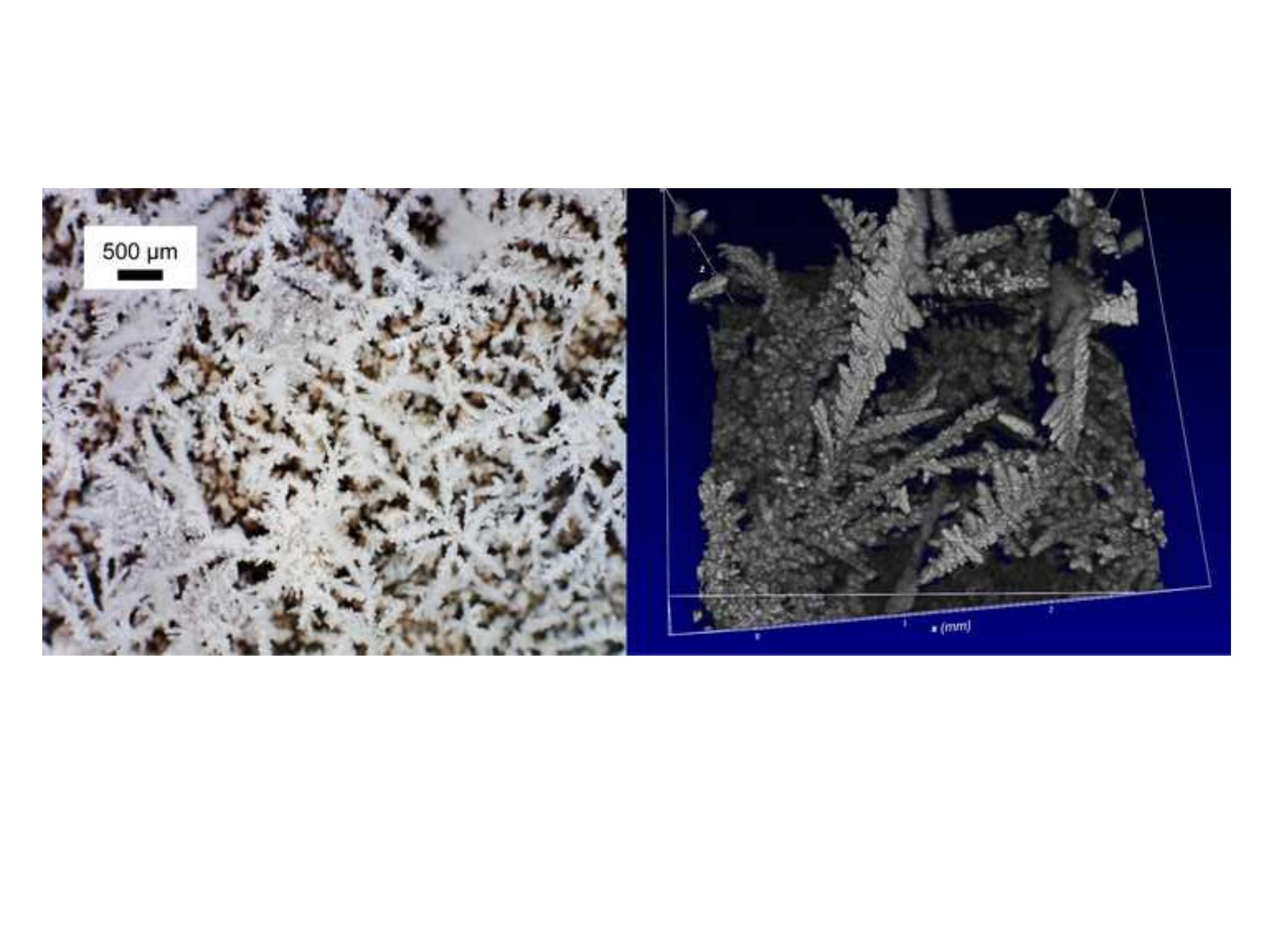

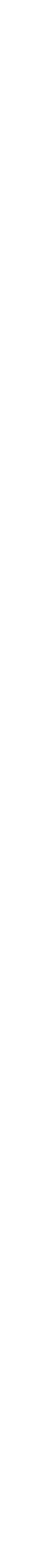




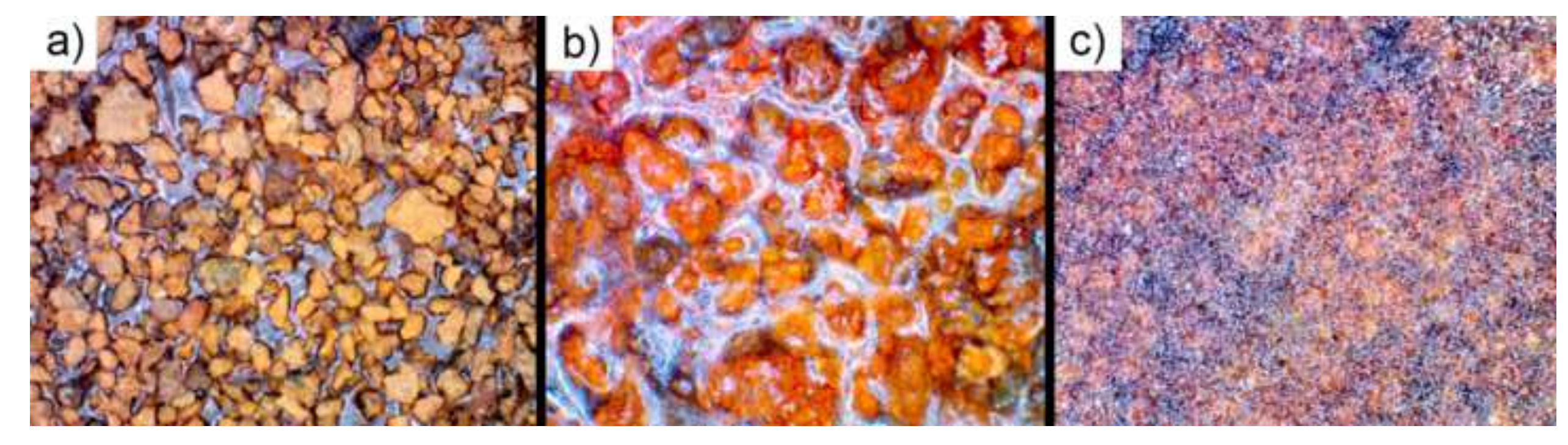



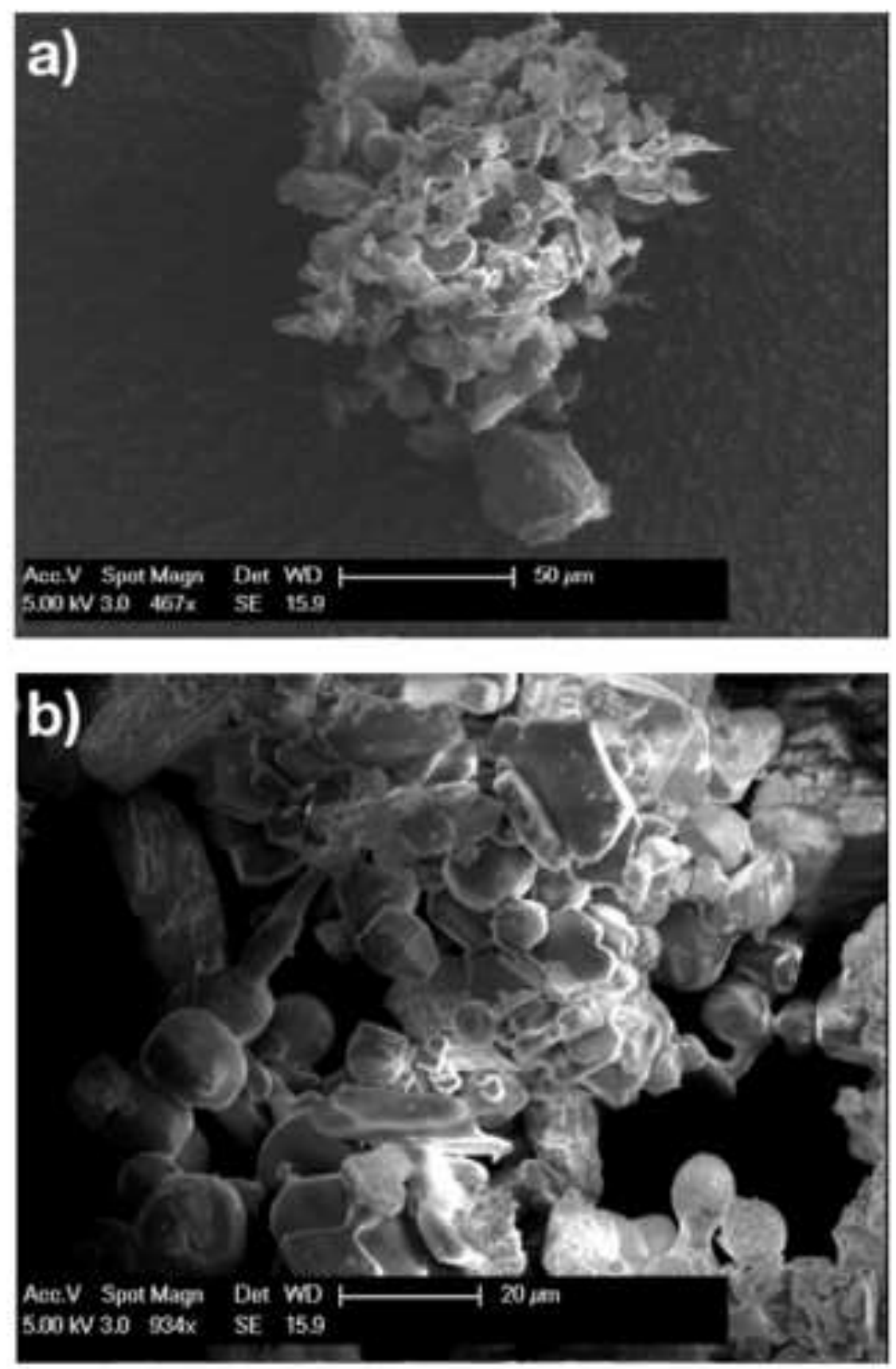

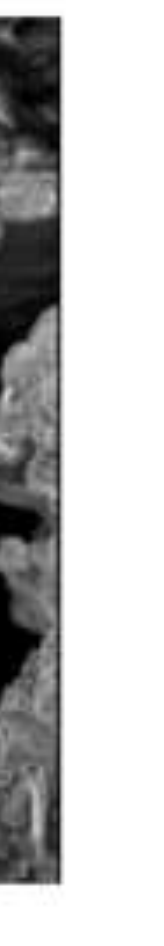

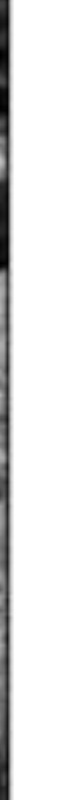



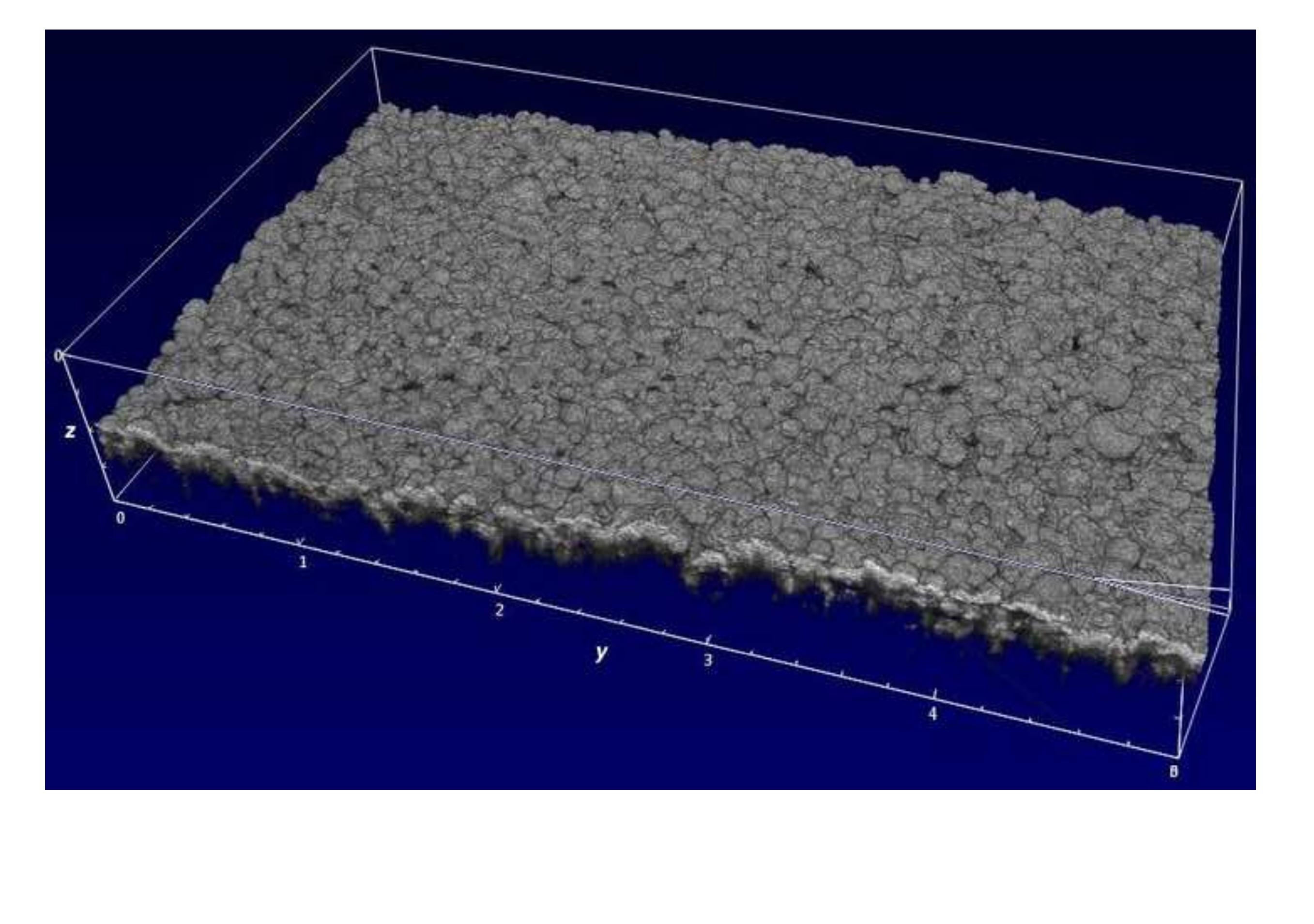

2010)



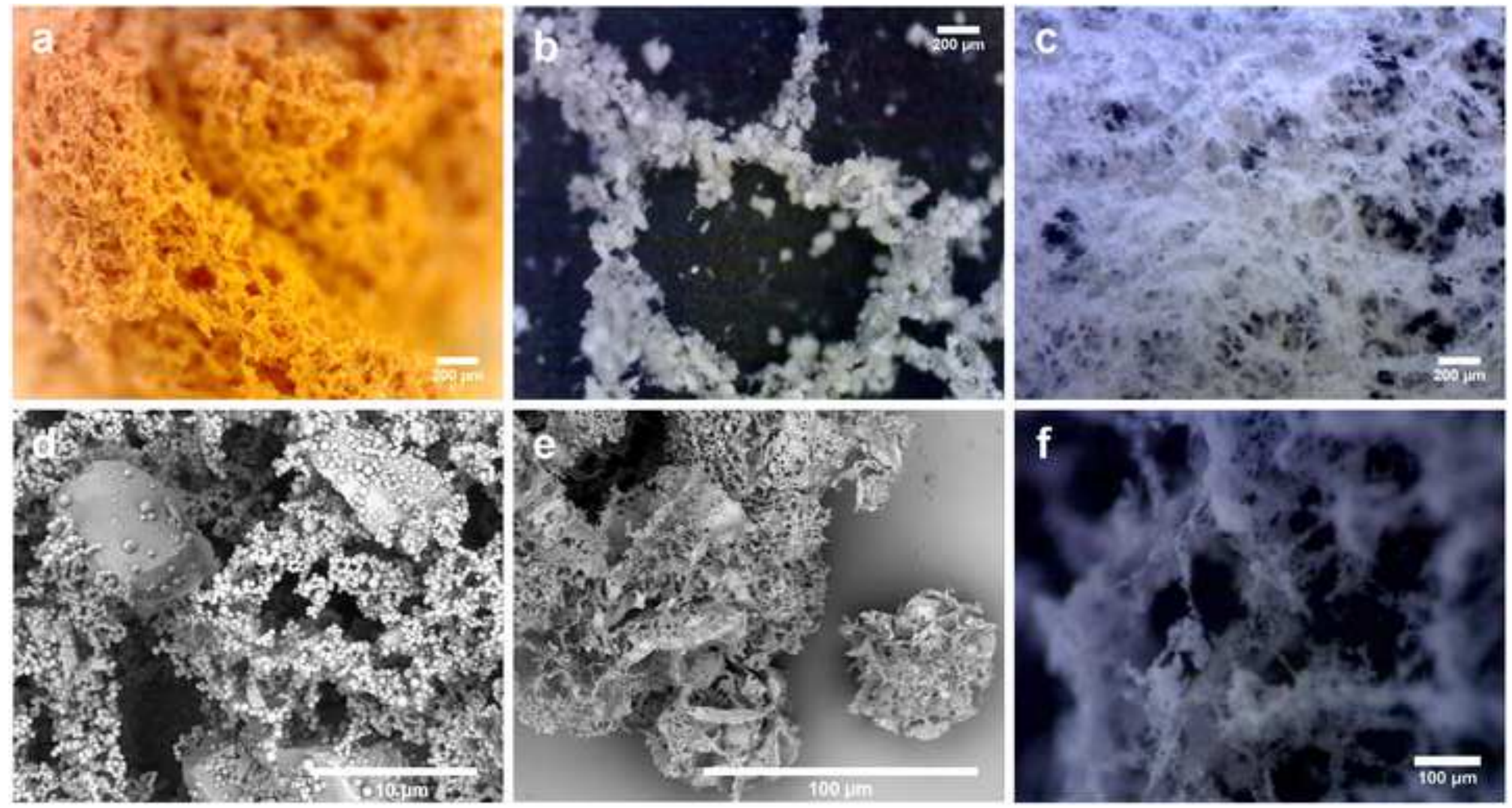


\section{Sublimation of ice-dust (organics and minerals)}

\section{intra-mixture}

(1)

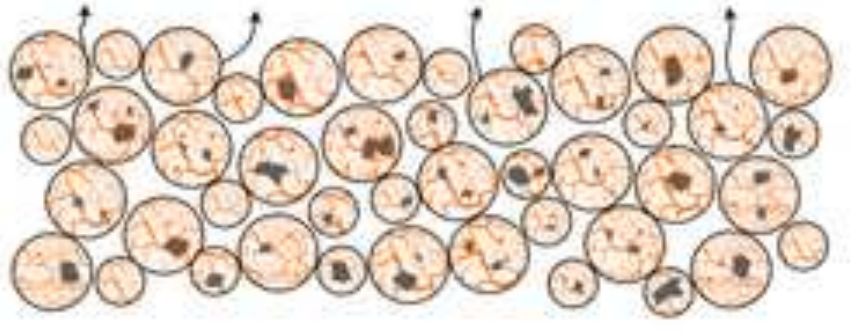

2

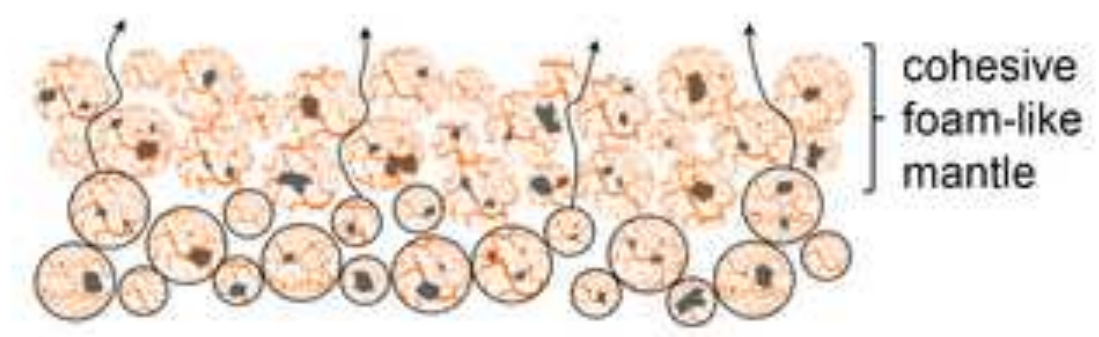

inter-mixture

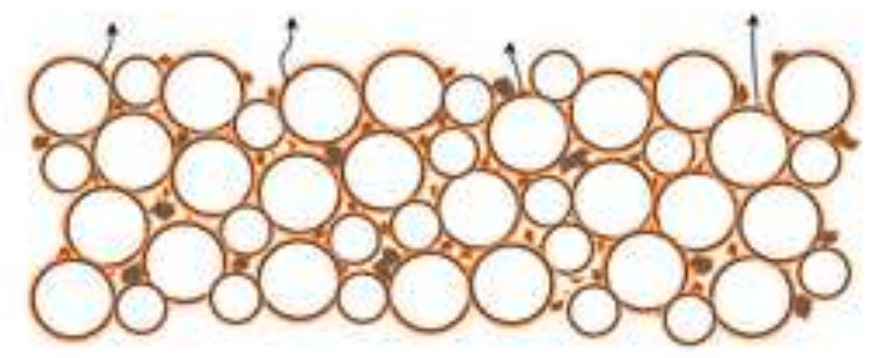

pressure build-up below the mantles

more restricted outward gas flow

$\rightarrow$ enhanced inward gas flow

$\rightarrow$ recondensation/sintering

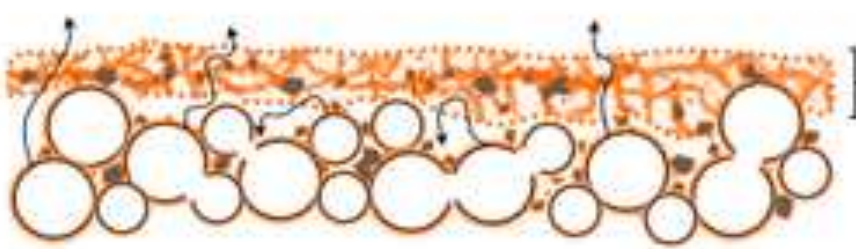

more

compact

\& less cohesive mantle

3

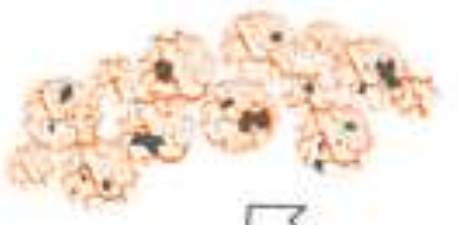

$\longleftarrow$ ejection of mantle fragments

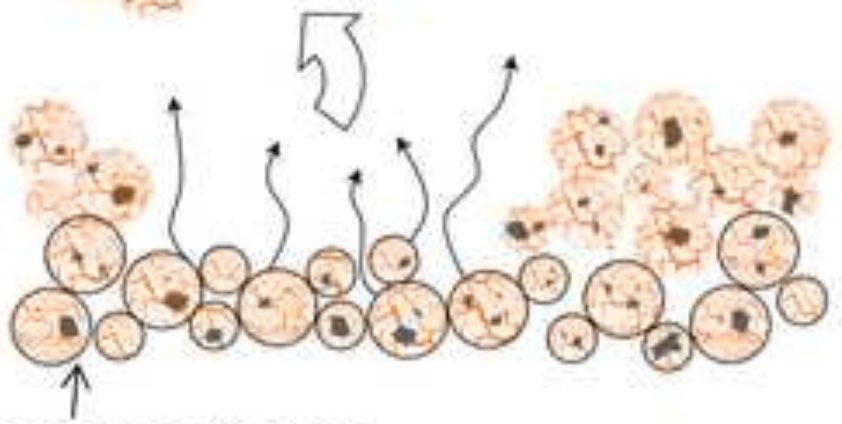

loose subsurface ice
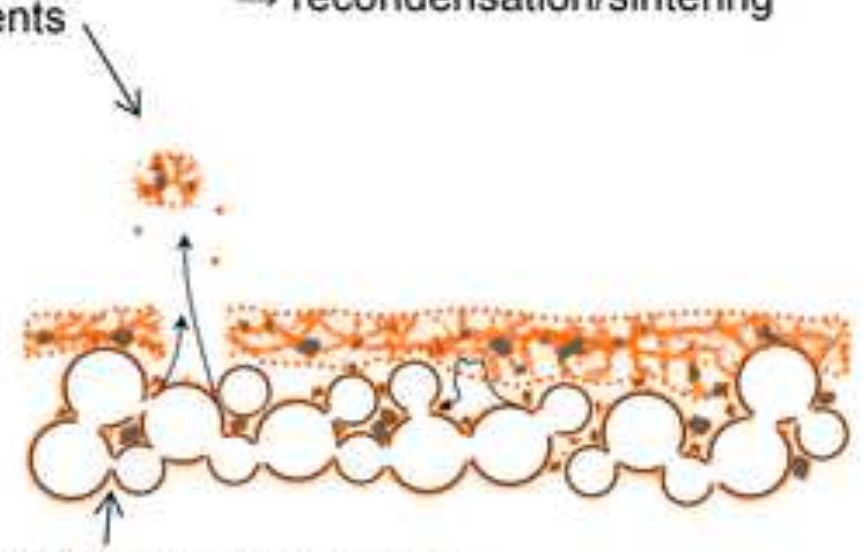

solidified subsurface ice 

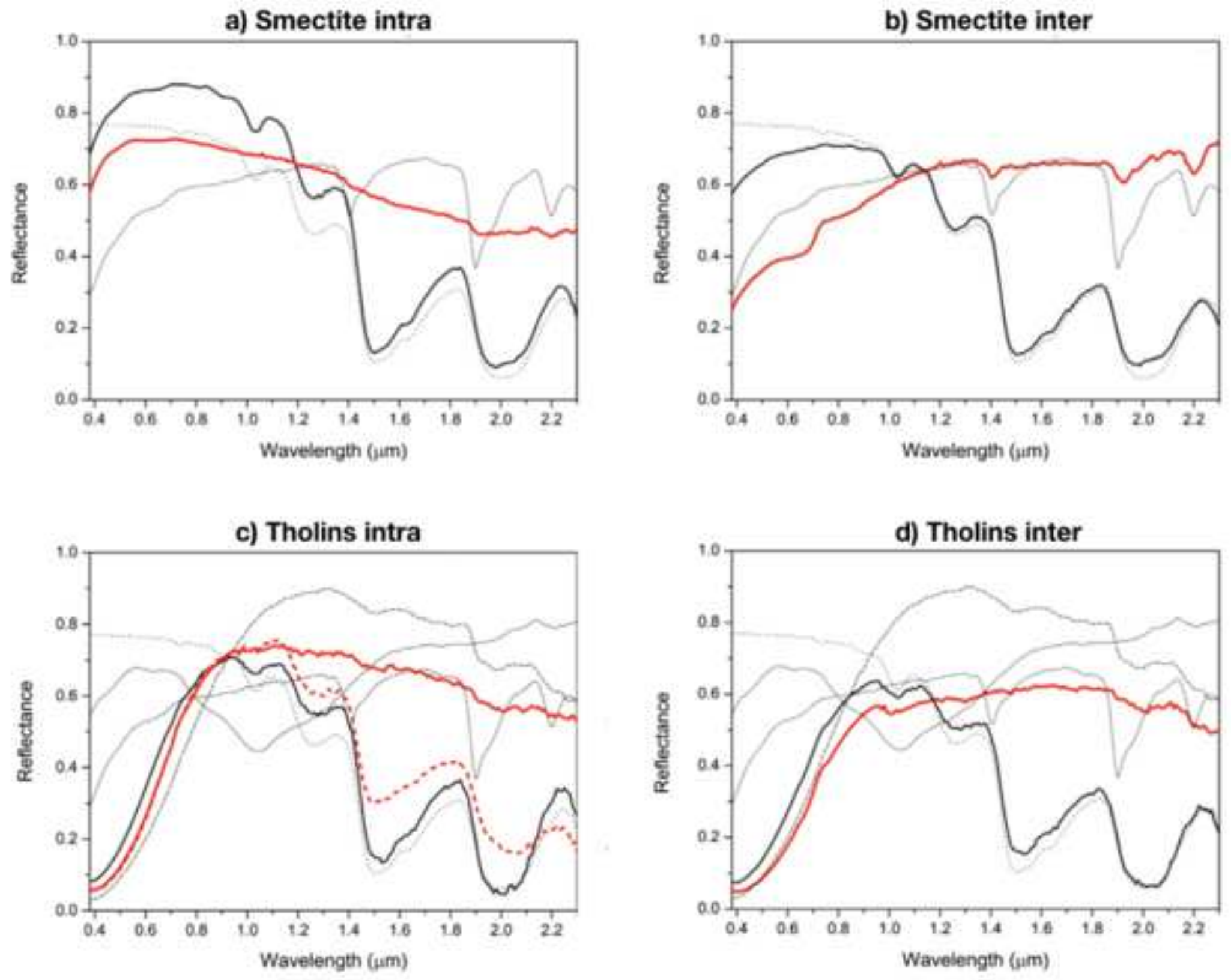
$\square \mathrm{cm}$
5
$\square$

a)

th

b)

\subsection{0h}

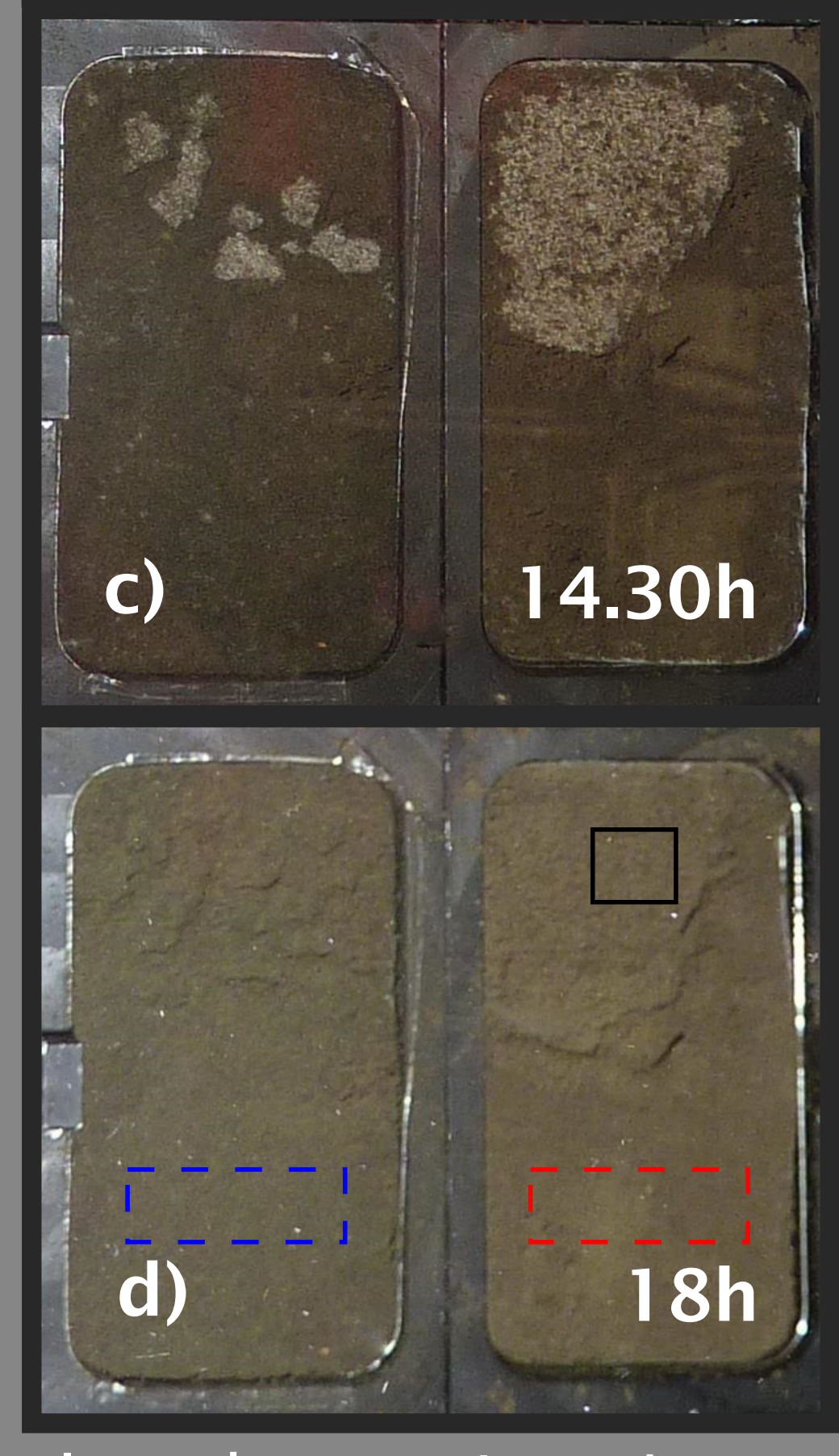

Inter-mixture Intra-mixture Experiment \#3 98\% water ice $1.8 \%$ charcoa

\section{Tholins}
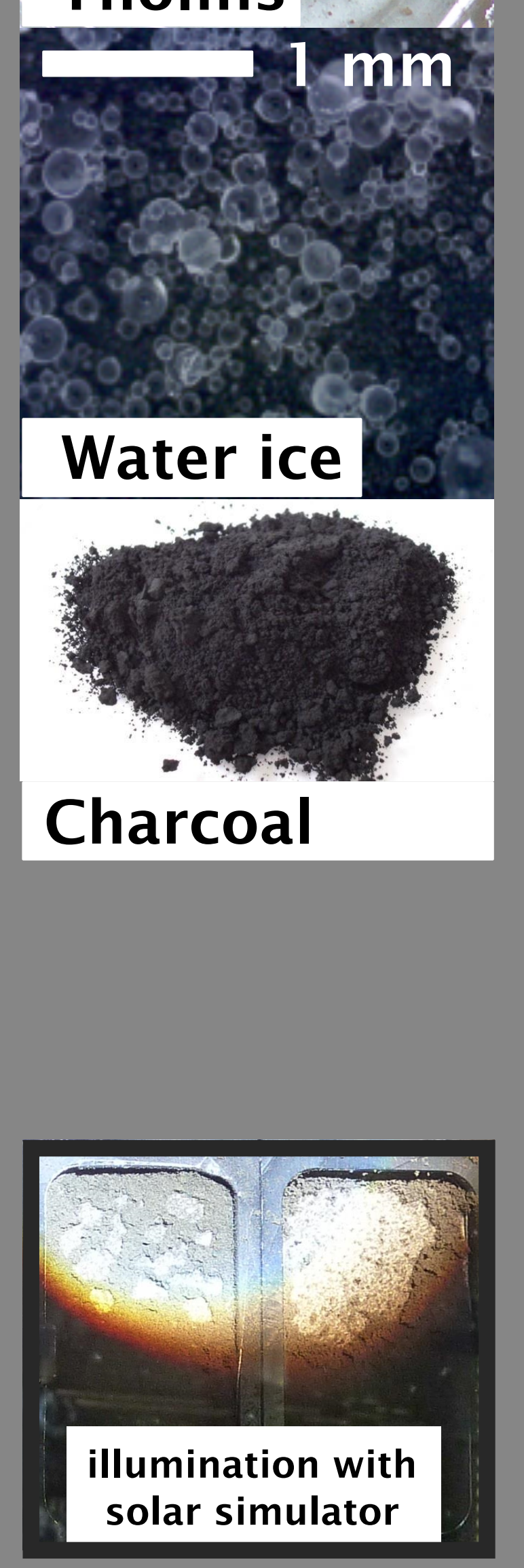

\section{Charcoal}

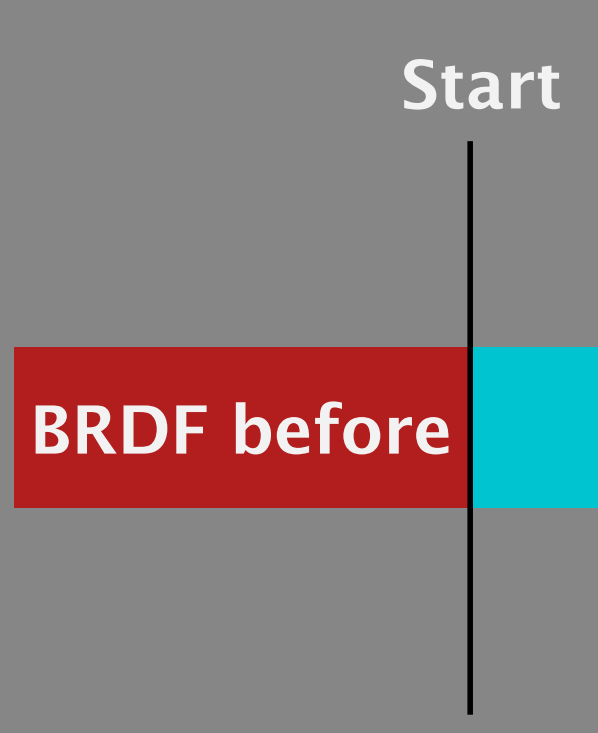

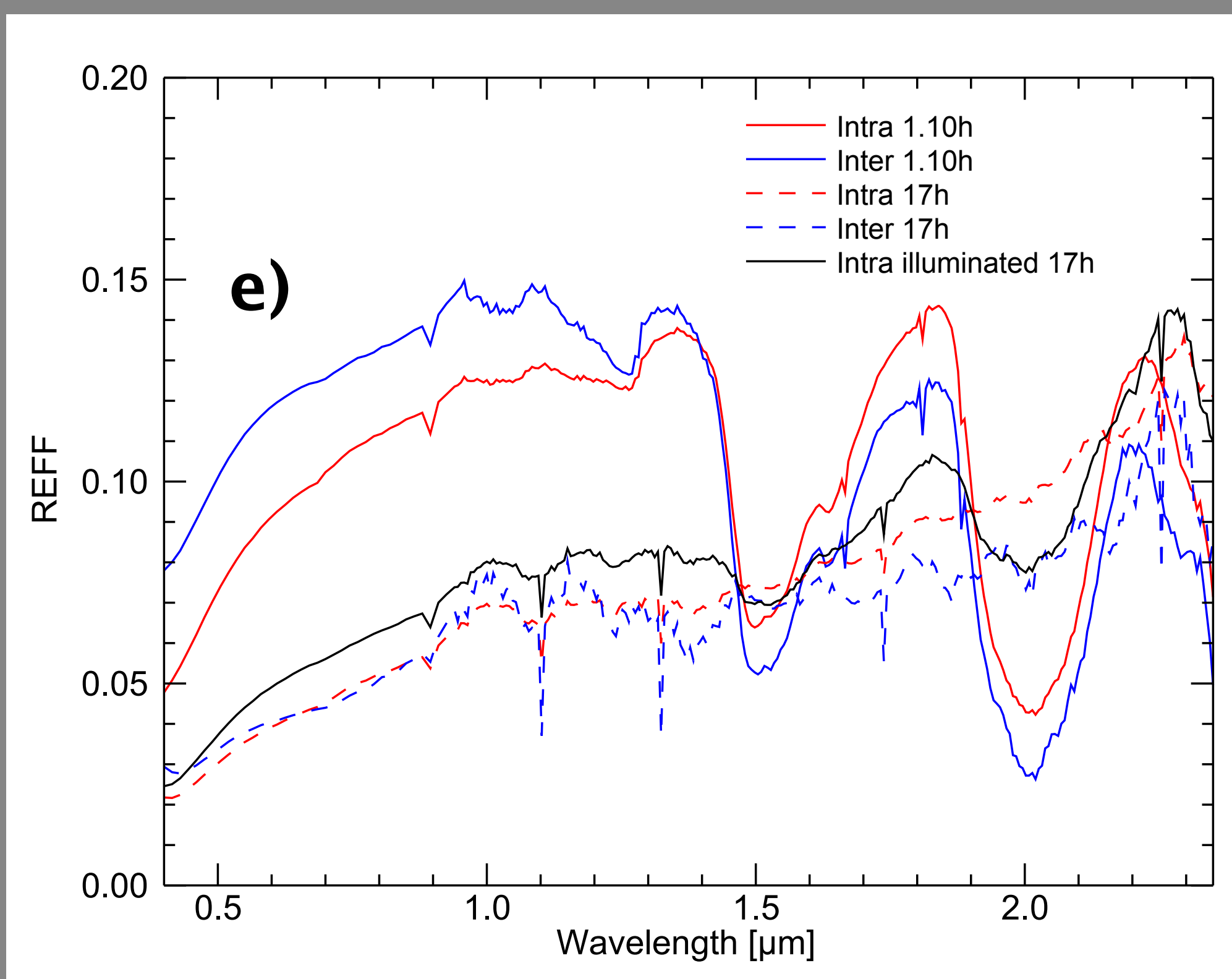
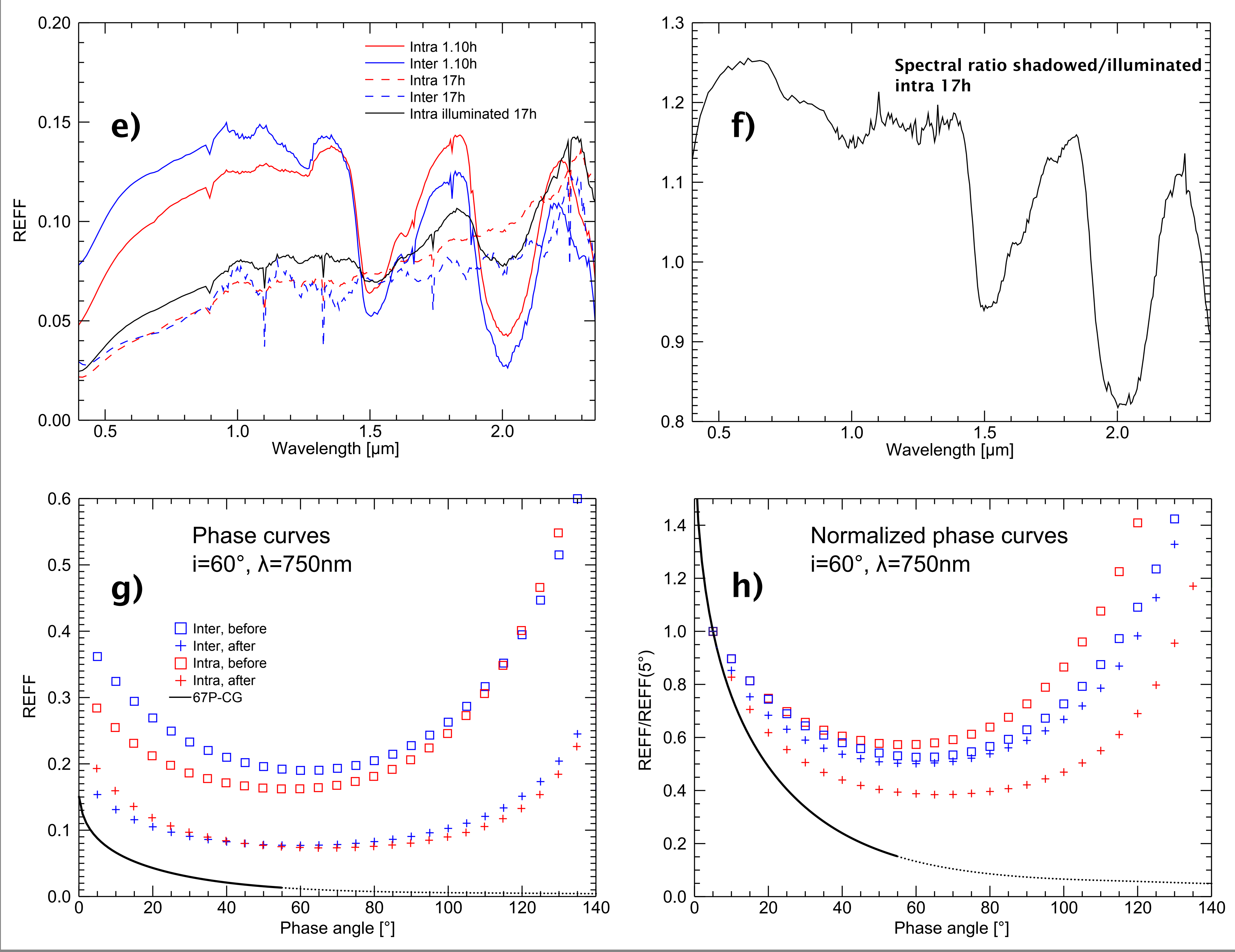

spectrum

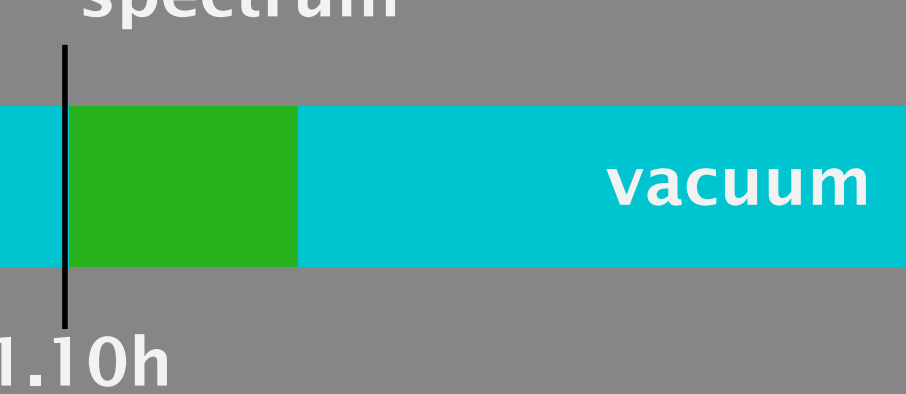

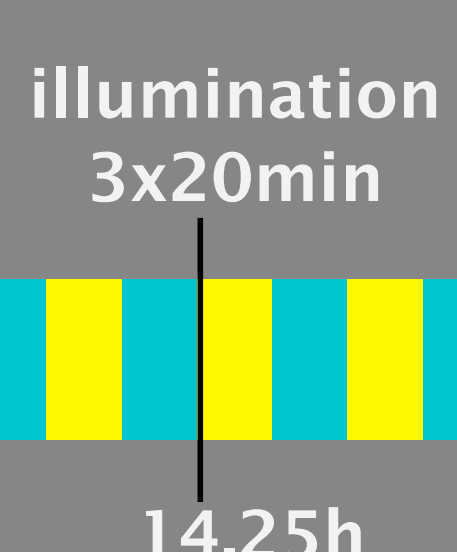

spectrum
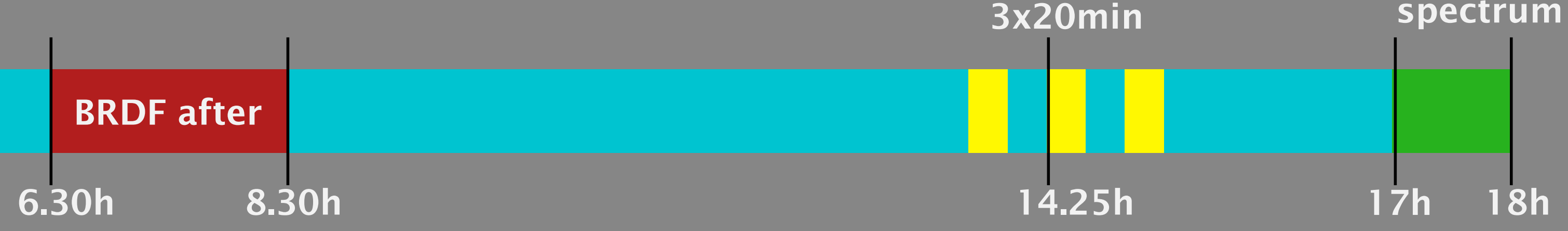


\section{Reflectance of JSC1-AF and water ice intimate mixtures.}
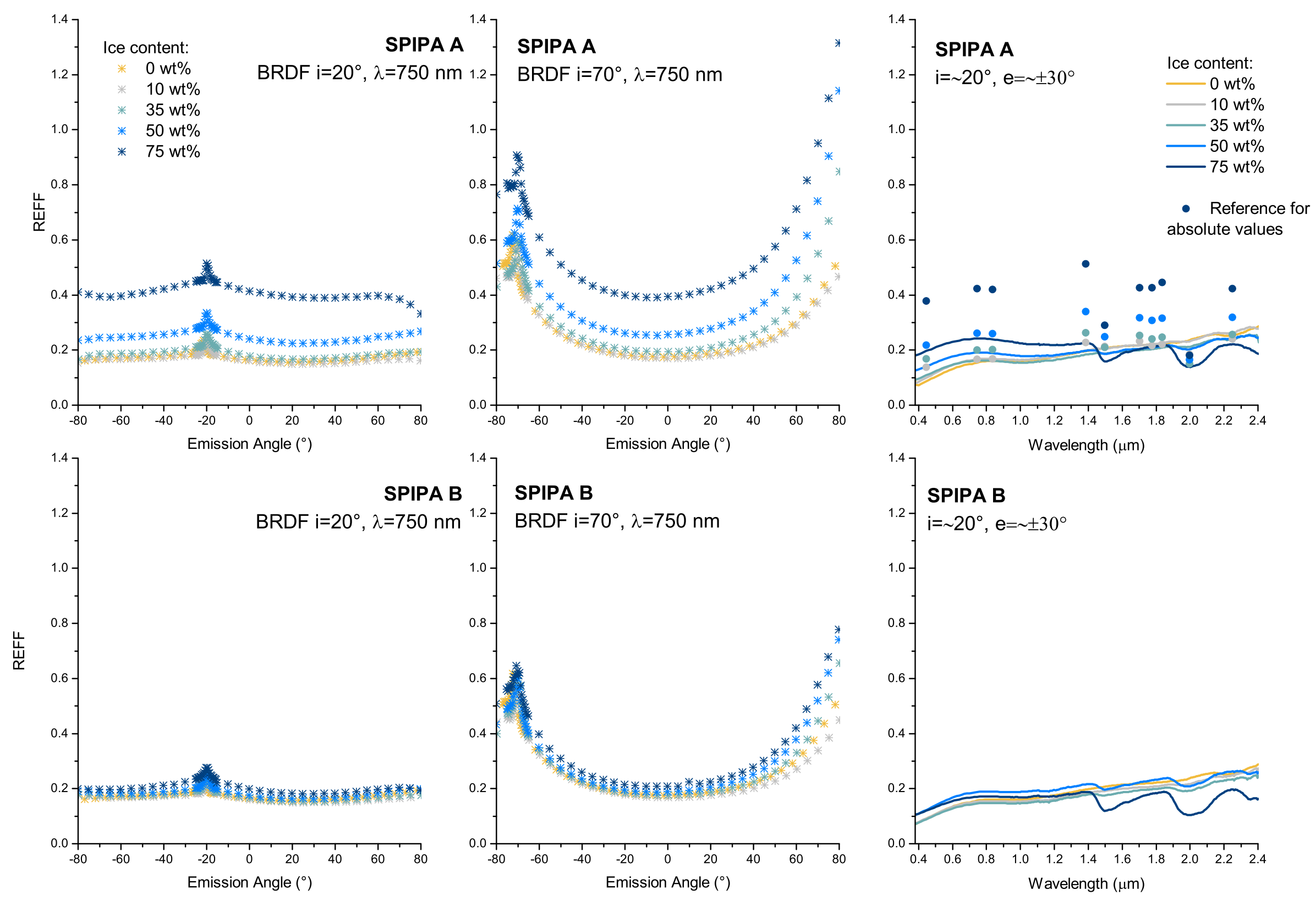
a)

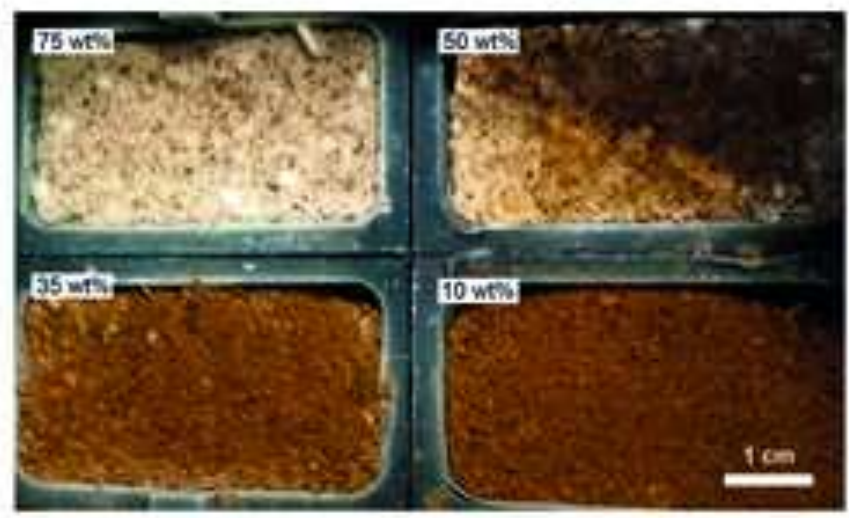

c)

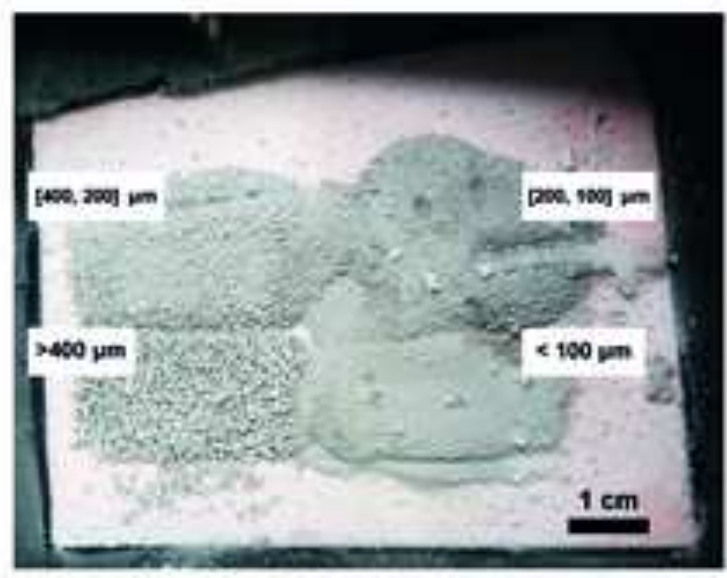

e)

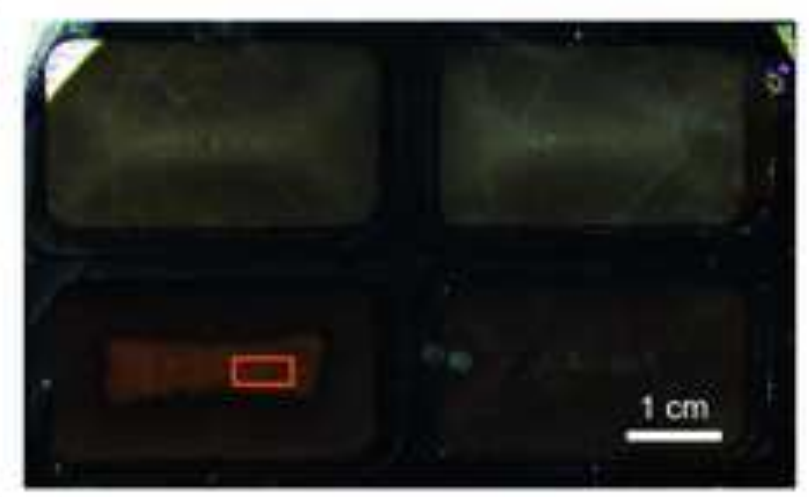

g)

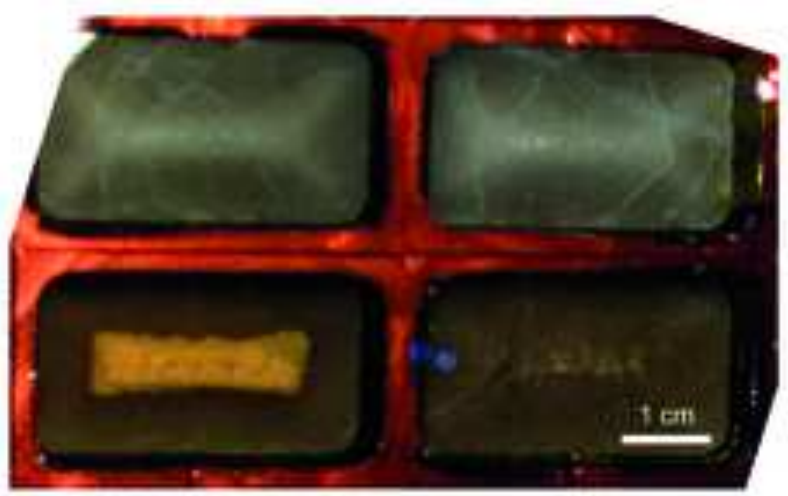

b)

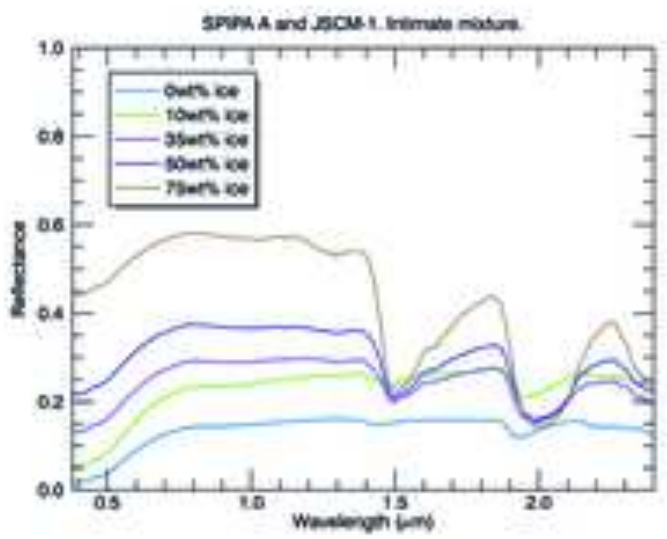

d)

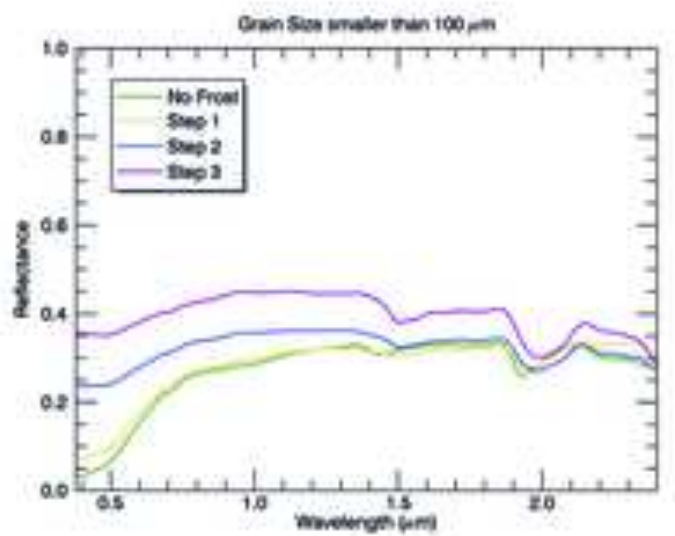

f)

h)
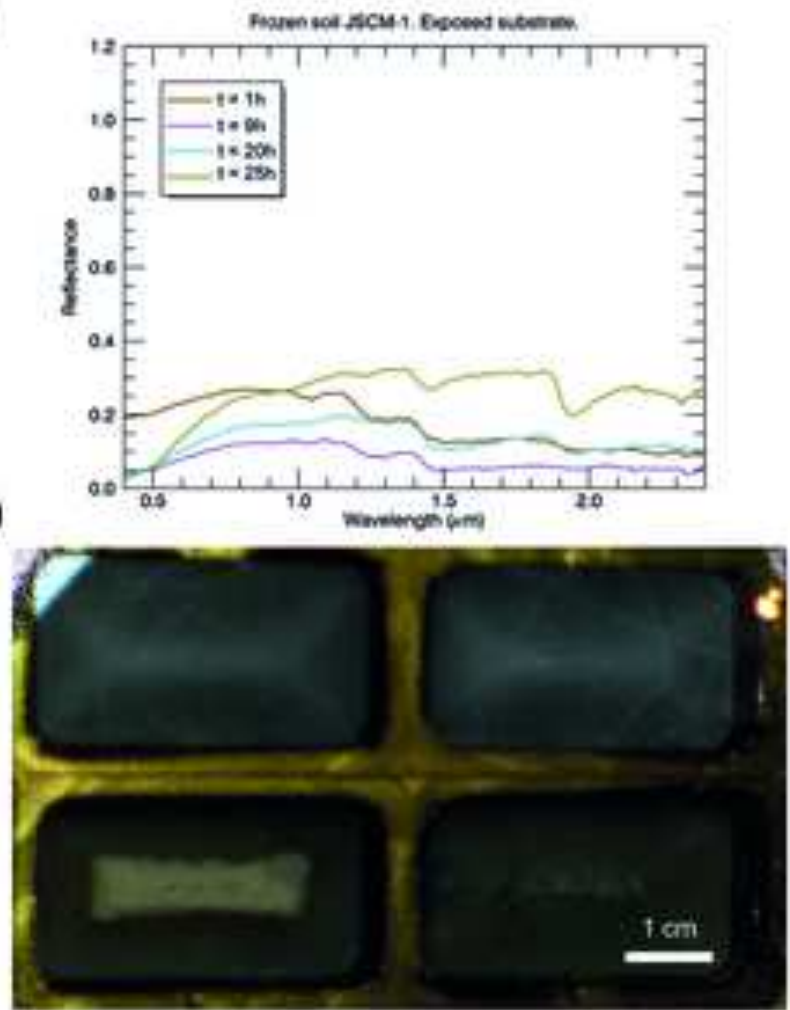


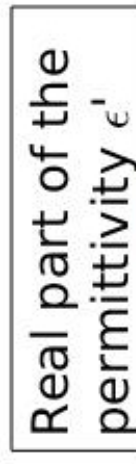
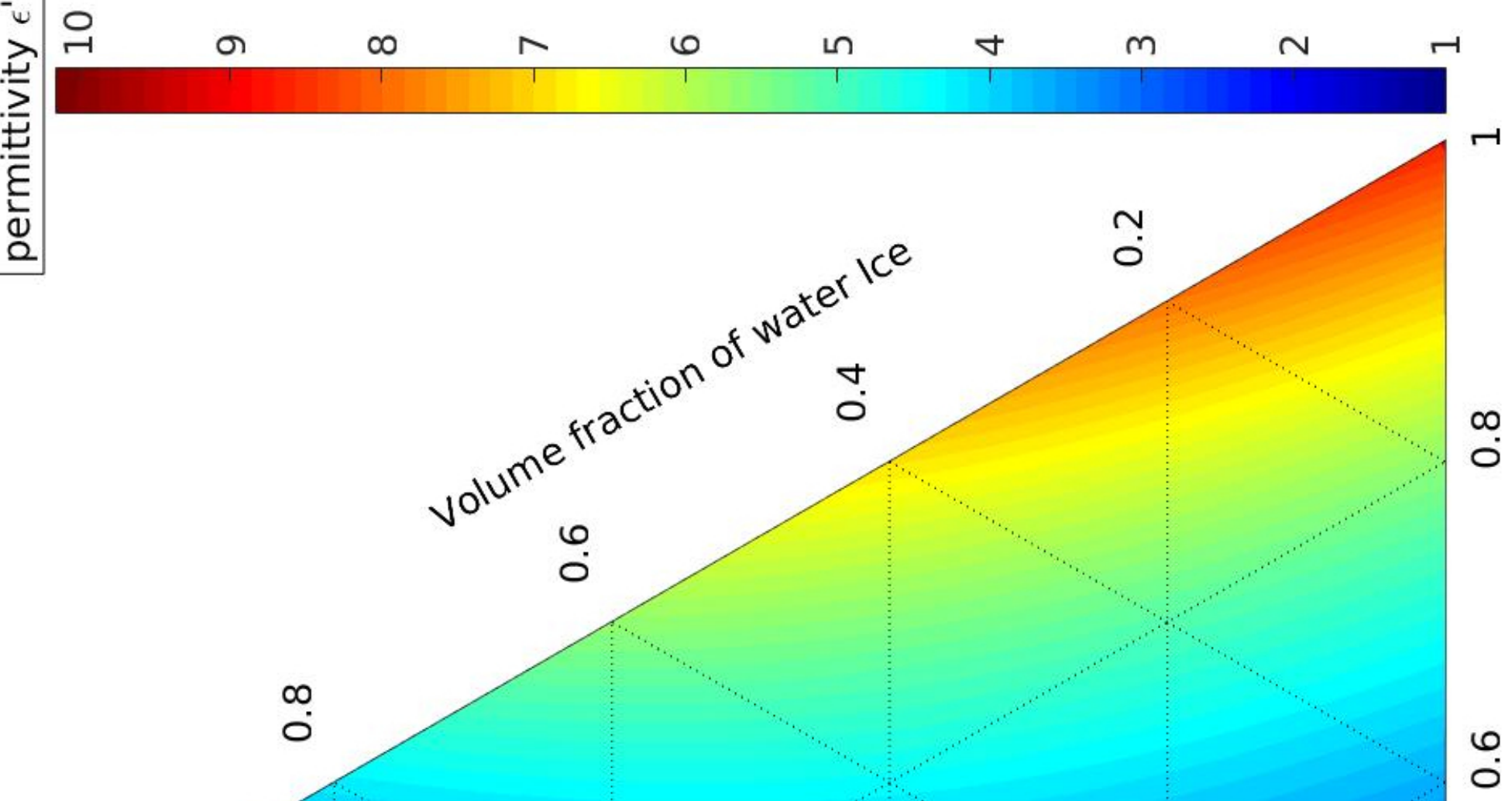

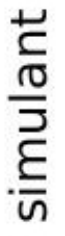

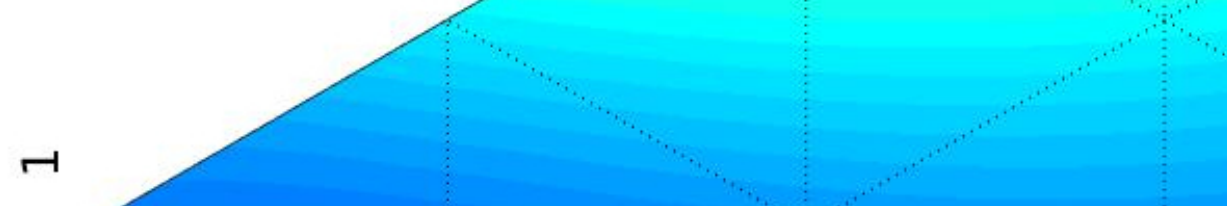

○
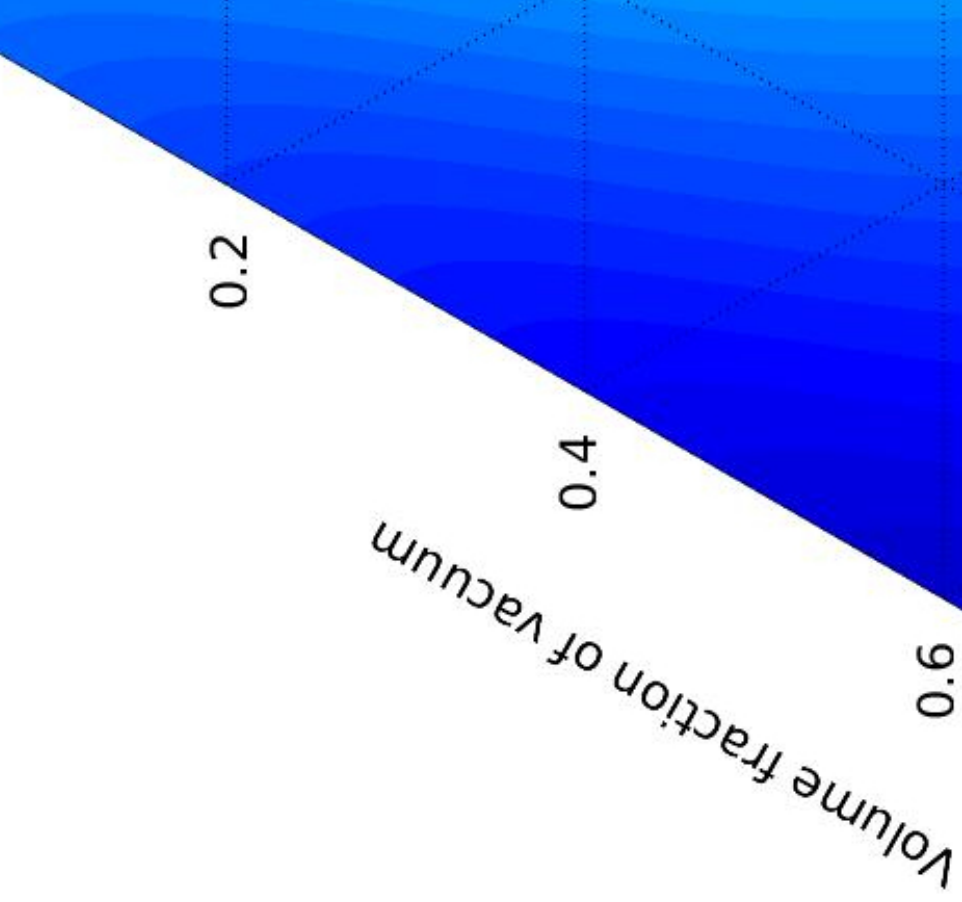

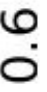

$\frac{1}{2}$

$\leqq$

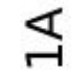

ن

系

4

$\dot{0}$

등 


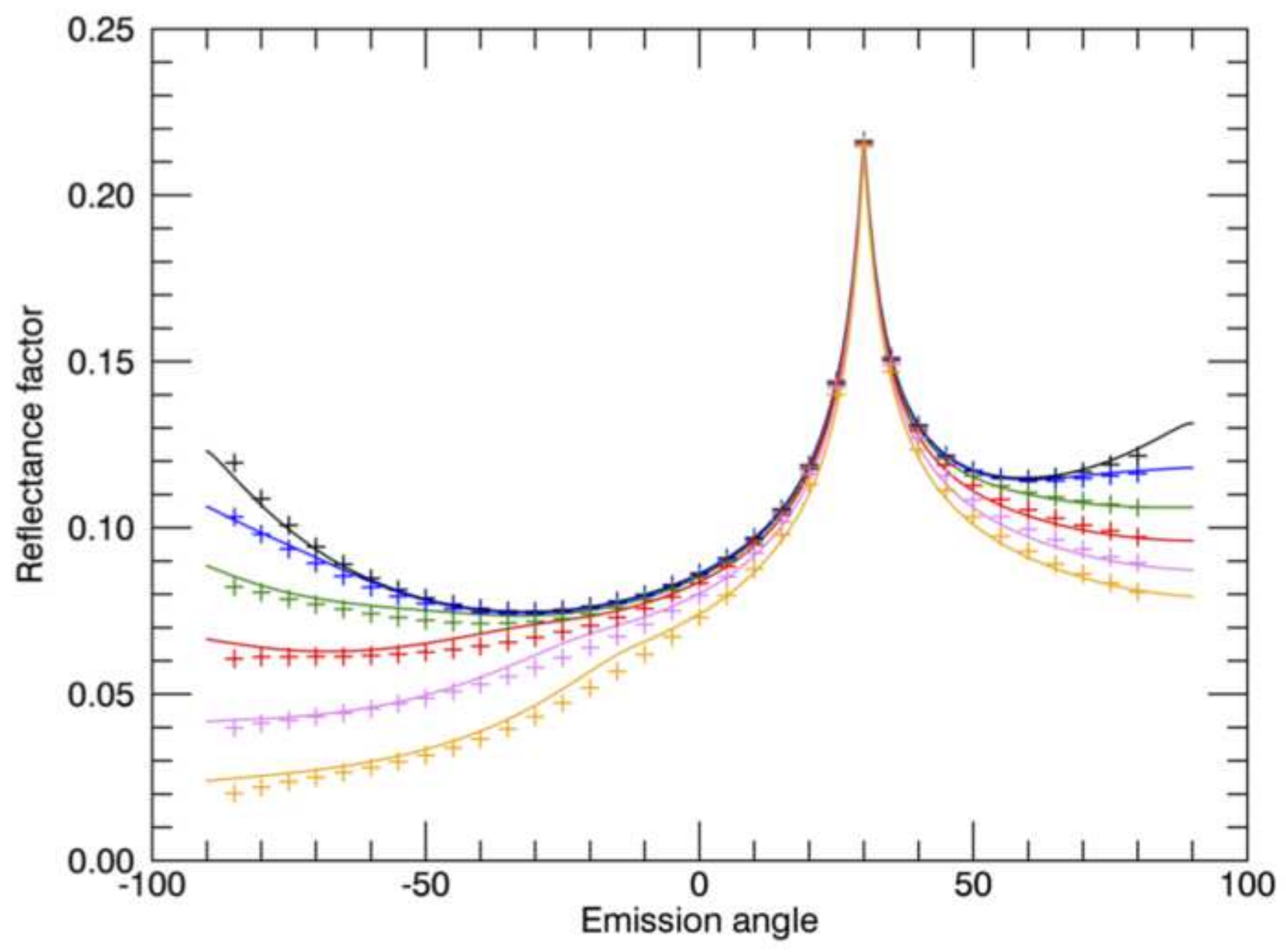




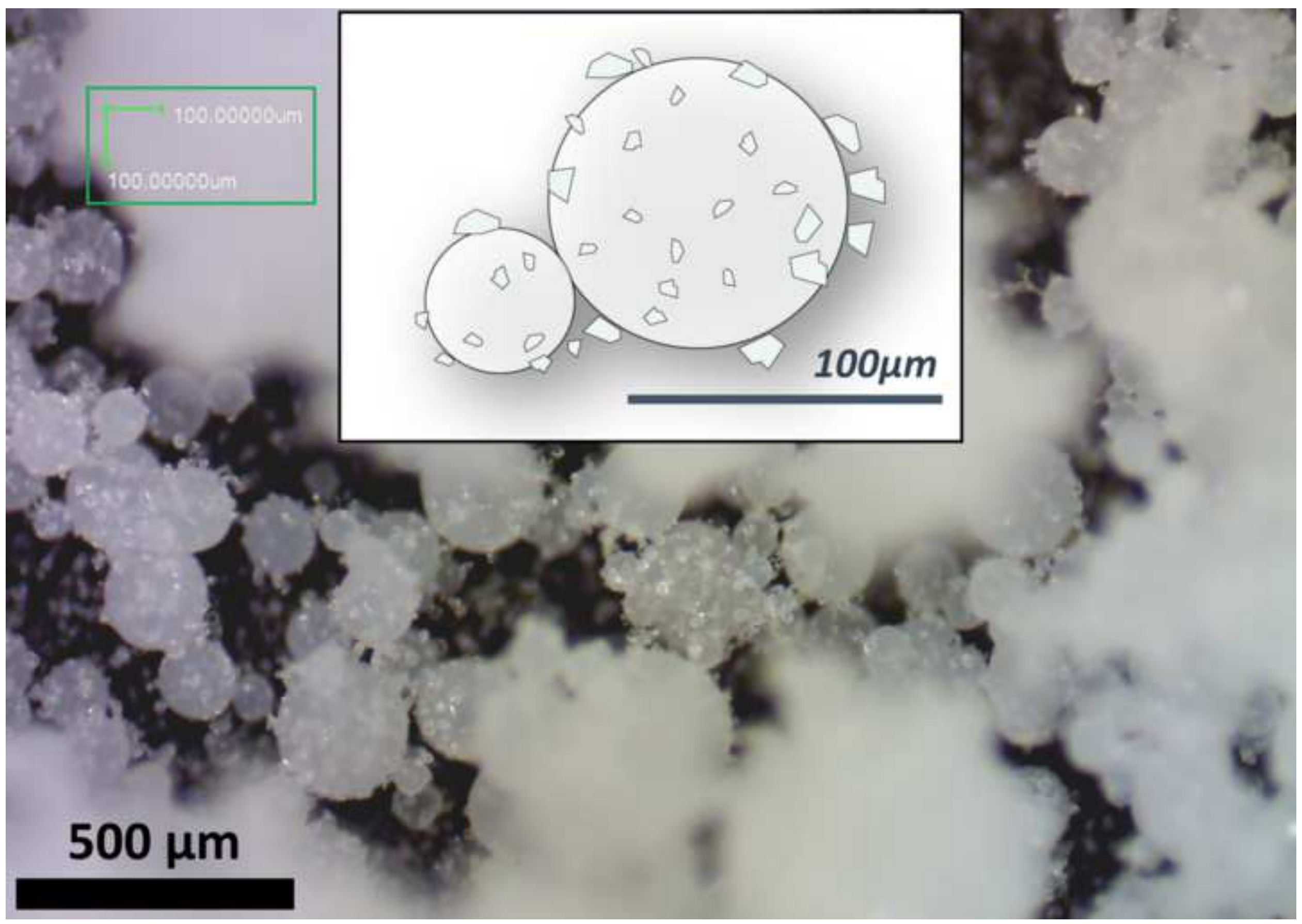




\begin{tabular}{|c|c|c|c|c|c|c|c|c|c|}
\hline Technique & $\begin{array}{l}\text { gamma- } \\
\text { ray / } \\
\text { Neutron }\end{array}$ & $\begin{array}{l}\text { UV } \\
\text { imaging }\end{array}$ & $\begin{array}{l}\text { Color } \\
\text { visible } \\
\text { imaging }\end{array}$ & $\begin{array}{l}\text { Laser al- } \\
\text { timetry }\end{array}$ & $\begin{array}{l}\text { VIS-NIR } \\
\text { spectral } \\
\text { imaging }\end{array}$ & $\begin{array}{l}\text { Thermal } \\
\text { infrared }\end{array}$ & $\begin{array}{l}\text { Micro- } \\
\text { wave }\end{array}$ & $\begin{array}{l}\text { Imaging } \\
\text { radar }\end{array}$ & $\begin{array}{l}\text { Sounding } \\
\text { radar }\end{array}$ \\
\hline $\begin{array}{l}\text { Wave- } \\
\text { length }\end{array}$ & $<10 \mathrm{pm}$ & $\begin{array}{l}10-400 \\
n m\end{array}$ & $\begin{array}{l}400- \\
1100 \mathrm{~nm}\end{array}$ & $1064 \mathrm{~nm}$ & $\begin{array}{l}0.4-5 \\
\mu \mathrm{m}\end{array}$ & $\begin{array}{l}5 \mu \mathrm{m}-1 \\
\mathrm{~mm}\end{array}$ & $\mathrm{~mm}-\mathrm{cm}$ & $\mathrm{cm}-\mathrm{m}$ & $\begin{array}{l}10 \mathrm{~m}-1 \\
\mathrm{~km}\end{array}$ \\
\hline $\begin{array}{l}\text { Pene- } \\
\text { tration }\end{array}$ & $\mathrm{m}$ & $\mu \mathrm{m}$ & $\mu \mathrm{m}-\mathrm{mm}$ & $\mu \mathrm{m}-\mathrm{mm}$ & $\mu \mathrm{m}-\mathrm{mm}$ & $\mathrm{cm}$ & $\mathrm{cm}$ & $\mathrm{m}$ & $\mathrm{km}$ \\
\hline $\begin{array}{l}\text { Typical } \\
\text { resolu- } \\
\text { tion from } \\
\text { orbit }\end{array}$ & $\begin{array}{l}10- \\
100 \mathrm{~km}\end{array}$ & $\mathrm{~km}$ & $<\mathrm{m}$ & $10 \mathrm{~m}$ & $10 \mathrm{~m}$ & $10 \mathrm{~m}$ & $10 \mathrm{~m}$ & $\mathrm{~m}$ & $\mathrm{~km}$ \\
\hline $\begin{array}{l}\text { Active / } \\
\text { passive }\end{array}$ & passive & passive & passive & active & passive & passive & $\begin{array}{l}\text { active / } \\
\text { passive }\end{array}$ & active & active \\
\hline $\begin{array}{l}\text { Main } \\
\text { advan- } \\
\text { tages }\end{array}$ & $\begin{array}{l}\text { Quanti- } \\
\text { fication } \\
\text { of hy- } \\
\text { drogen } \\
\text { in the } \\
\text { shallow } \\
\text { subsur- } \\
\text { face }\end{array}$ & $\begin{array}{l}\text { Detection } \\
\text { of low } \\
\text { amounts } \\
\text { of water }\end{array}$ & $\begin{array}{l}\text { Detection } \\
\text { of water } \\
\text { and ice at } \\
\text { highest } \\
\text { resolu- } \\
\text { tion }\end{array}$ & $\begin{array}{l}\text { Observa- } \\
\text { tion } \\
\text { possible } \\
\text { in } \\
\text { perma- } \\
\text { nently } \\
\text { shad- } \\
\text { owed } \\
\text { areas }\end{array}$ & $\begin{array}{l}\text { Physical } \\
\text { state of } \\
\text { water, } \\
\text { particle } \\
\text { size, } \\
\text { temper- } \\
\text { ature of } \\
\text { ice. } \\
\text { Quan- } \\
\text { tifica- } \\
\text { tion } \\
\text { possible }\end{array}$ & $\begin{array}{l}\text { Very } \\
\text { sensitive } \\
\text { to low } \\
\text { amounts } \\
\text { of water. } \\
\text { Quan- } \\
\text { tifica- } \\
\text { tion } \\
\text { possible }\end{array}$ & $\begin{array}{l}\text { Sensitive } \\
\text { to ice } \\
\text { and } \\
\text { liquid } \\
\text { water, } \\
\text { texture } \\
\text { and } \\
\text { rough- } \\
\text { ness }\end{array}$ & $\begin{array}{l}\text { Imaging } \\
\text { of the } \\
\text { shallow } \\
\text { subsur- } \\
\text { face. } \\
\text { Not } \\
\text { a dected } \\
\text { by the } \\
\text { atmo- } \\
\text { sphere }\end{array}$ & $\begin{array}{l}\text { Very } \\
\text { deep } \\
\text { sound- } \\
\text { ing in } \\
\text { ice. Ex- } \\
\text { tremely } \\
\text { high } \\
\text { sensitiv- } \\
\text { ity to } \\
\text { liquid } \\
\text { water }\end{array}$ \\
\hline $\begin{array}{l}\text { Recent } \\
\text { example }\end{array}$ & $\begin{array}{l}\text { GRS/ } \\
\text { Odyssey }\end{array}$ & $\begin{array}{l}\text { Alice/ } \\
\text { Rosetta }\end{array}$ & $\begin{array}{l}\text { HiRISE/ } \\
\text { MRO }\end{array}$ & $\begin{array}{l}\text { MLA/ } \\
\text { Messen- } \\
\text { ger }\end{array}$ & $\begin{array}{l}\text { OMEGA/ } \\
\text { Mex }\end{array}$ & $\begin{array}{l}\text { THEMIS } \\
\text { /Odyssey }\end{array}$ & $\begin{array}{l}\text { MIRO } \\
\text { /Rosetta }\end{array}$ & $\begin{array}{l}\text { Radar } \\
\text { /Cassini }\end{array}$ & $\begin{array}{l}\text { MARSIS } \\
\text { /Mex }\end{array}$ \\
\hline
\end{tabular}

Table 1: A summary of different remote-sensing techniques based on the interaction between the regolith and electromagnetic field at different wavelengths over the entire spectrum. 


\begin{tabular}{|c|c|c|c|}
\hline Parameter & Symbol & Description & Comment \\
\hline Single scattering albedo & $\omega$ & $\begin{array}{l}\text { Ratio between the total } \\
\text { scattered radiation and the total } \\
\text { absorbed radiation by one } \\
\text { particle }\end{array}$ & \\
\hline $\begin{array}{l}\text { Single scattering phase } \\
\text { function }\end{array}$ & $(b, c)$ & $\begin{array}{l}\text { Angular distribution of the } \\
\text { light scattered by one isolated } \\
\text { particle }\end{array}$ & $\begin{array}{l}\text { Various formulations can be } \\
\text { used. A popular one is the 2- } \\
\text { terms Henyey-Greenstein } \\
\text { function. }\end{array}$ \\
\hline Mean slope angle & $\bar{\theta}$ & $\begin{array}{l}\text { Quantifies the roughness of the } \\
\text { surface. }\end{array}$ & $\begin{array}{l}\text { The slope distribution is } \\
\text { assumed to be isotropic }\end{array}$ \\
\hline Porosity & $\mathrm{p}$ & $\begin{array}{l}\text { The bulk porosity of the } \\
\text { sample }\end{array}$ & Introduced in 2008 \\
\hline $\begin{array}{l}\text { Shadow Hiding } \\
\text { Opposition Effect } \\
\text { (SHOE) }\end{array}$ & $\mathrm{B}_{\mathrm{s}}, \mathrm{h}_{\mathrm{s}}$ & $\begin{array}{l}\text { Opposition effect due to the } \\
\text { self-shadowing of particles in } \\
\text { the sample. Described by the } \\
\text { amplitude and width of the } \\
\text { peak. }\end{array}$ & No dependence on wavelength \\
\hline $\begin{array}{l}\text { Coherent Backscattering } \\
\text { Opposition Effect } \\
\text { (CBOE) }\end{array}$ & $\mathrm{B}_{\mathrm{c}}, \mathrm{h}_{\mathrm{c}}$ & $\begin{array}{l}\text { Opposition effect due to } \\
\text { coherent interactions between } \\
\text { photons scattered along the } \\
\text { same path in opposite } \\
\text { directions. Described by the } \\
\text { amplitude and width of the } \\
\text { peak. }\end{array}$ & $\begin{array}{c}\text { Dependent on wavelength. See } \\
\text { Hapke, } 2002 .\end{array}$ \\
\hline
\end{tabular}

Table 2: Parameters of the Hapke model. See Figure 1 for illustrations of the definition and role of the parameter. 


\begin{tabular}{|c|c|c|c|c|c|c|}
\hline Acronym & Full Name & Description & $\begin{array}{l}\text { Wavelength } \\
\text { / Spectral } \\
\text { range }\end{array}$ & $\begin{array}{l}\text { Tempera } \\
\text { ture } \\
\text { range }\end{array}$ & $\begin{array}{l}\text { Pressure } \\
\text { conditions }\end{array}$ & $\begin{array}{l}\text { Notable } \\
\text { capabilities }\end{array}$ \\
\hline PHIRE-2 & $\begin{array}{l}\text { PHysikalisches Institut } \\
\text { Reflectance Experiment } \\
-2\end{array}$ & $\begin{array}{l}\text { A radio-goniometer used } \\
\text { to measure the } \\
\text { bidirectional reflectance } \\
\text { of icy samples as a } \\
\text { function of the } \\
\text { incidence, emission and } \\
\text { phase angle }\end{array}$ & $\begin{array}{c}400-1100 \\
\mathrm{~nm}\end{array}$ & $\begin{array}{l}240 \mathrm{~K}- \\
\text { ambient }\end{array}$ & $\begin{array}{l}\text { Ambient } \\
\text { pressure }\end{array}$ & $\begin{array}{c}\text { Optional setup } \\
\text { for measurements } \\
\text { at low phase } \\
\text { angle }\end{array}$ \\
\hline SCITEAS & $\begin{array}{c}\text { Simulation Chamber for } \\
\text { Imaging the Temporal } \\
\text { Evolution of Analogue } \\
\text { Samples }\end{array}$ & $\begin{array}{l}\text { Simulation chamber } \\
\text { equipped with a } \\
\text { hyperspectral imaging } \\
\text { system }\end{array}$ & $\begin{array}{c}380-2400 \\
\mathrm{~nm}\end{array}$ & $\begin{array}{l}100 \mathrm{~K}- \\
\text { ambient }\end{array}$ & $\begin{array}{c}10^{-6} \mathrm{mbar}-1 \\
\mathrm{bar}\end{array}$ & $\begin{array}{l}\text { Designed for long } \\
\text { sublimation } \\
\text { experiments }\end{array}$ \\
\hline POLICES & $\begin{array}{l}\text { POLarimeter for ICE } \\
\text { Samples }\end{array}$ & $\begin{array}{l}\text { Full-stokes polarimetry } \\
\text { with variable incidence } \\
\text { angle }\end{array}$ & $400-800 \mathrm{~nm}$ & $\begin{array}{c}77 \mathrm{~K}- \\
\text { ambient }\end{array}$ & $\begin{array}{l}\text { Ambient } \\
\text { pressure }\end{array}$ & $\begin{array}{l}\text { An integrating } \\
\text { sphere is used for } \\
\text { high-sensitivity } \\
\text { characterizations } \\
\text { of the circular } \\
\text { polarization }\end{array}$ \\
\hline OCTOPUS & $\begin{array}{l}\text { Optical Coherence } \\
\text { Tomography Of } \\
\text { Planetary Ultracold } \\
\text { Samples }\end{array}$ & $\begin{array}{l}\text { Commercial OCT } \\
\text { instrument (Thorlabs } \\
\text { Ganymede) operated } \\
\text { with icy samples } \\
\text { through a quartz window }\end{array}$ & $930 \mathrm{~nm}$ & $\begin{array}{c}77 \mathrm{~K}- \\
\text { ambient }\end{array}$ & $\begin{array}{l}\text { Ambient } \\
\text { pressure }\end{array}$ & $\begin{array}{c}\text { 3D imaging of } \\
\text { the surface and } \\
\text { subsurface of the } \\
\text { samples }\end{array}$ \\
\hline Dielectric & Epsimu & $\begin{array}{c}\text { Coaxial cell to measure } \\
\text { the real and imaginary } \\
\text { part of the dielectric } \\
\text { constant }\end{array}$ & $\begin{array}{l}50 \mathrm{MHz}-\& \\
\mathrm{GHz}\end{array}$ & $\begin{array}{l}240 \mathrm{~K}- \\
\text { ambient }\end{array}$ & $\begin{array}{l}\text { Ambient } \\
\text { pressure }\end{array}$ & $\begin{array}{l}\text { Measurements of } \\
\text { porous ice/dust } \\
\text { mixtures at low } \\
\text { temperature }\end{array}$ \\
\hline MEFISTO & $\begin{array}{l}\text { MEsskammer für } \\
\text { FlugzeitInStrumente und } \\
\text { Time-Of-Flight }\end{array}$ & $\begin{array}{c}\text { Simulation chamber } \\
\text { equipped with a } \\
\text { hyperspectral imaging } \\
\text { system }\end{array}$ & $\begin{array}{c}380-2400 \\
\mathrm{~nm}\end{array}$ & $\begin{array}{l}100 \mathrm{~K}- \\
\text { ambient }\end{array}$ & $\begin{array}{c}10^{-7} \mathrm{mbar}-1 \\
\mathrm{bar}\end{array}$ & $\begin{array}{l}\text { Bombardment of } \\
\text { the sample } \\
\text { surface by } \\
\text { electrons and ions }\end{array}$ \\
\hline
\end{tabular}

Table 3: Instruments and facilities developed and/or used in the Ice Laboratory and by collaborators. 


\begin{tabular}{|c|c|c|c|c|c|c|c|c|}
\hline Setup & $\begin{array}{l}\text { Particl } \\
\text { es } \\
\text { produc } \\
\text { ed by }\end{array}$ & $\begin{array}{l}\text { Particl } \\
\text { es } \\
\text { freezin } \\
\mathrm{g} \text { in }\end{array}$ & $\begin{array}{l}\text { Condensa } \\
\text { tion } \\
\text { temperatu } \\
\text { re }\end{array}$ & $\begin{array}{l}\text { Particle } \\
\text { shape }\end{array}$ & Porosity & $\begin{array}{l}\text { Particle } \\
\text { size }\end{array}$ & $\begin{array}{l}\text { Contaminants } \\
\text { in intra- } \\
\text { mixtures }\end{array}$ & $\begin{array}{l}\text { Productio } \\
\text { n rate }\end{array}$ \\
\hline SPIPA-A & $\begin{array}{l}\text { ultraso } \\
\text { unds }\end{array}$ & air & $\sim 200 \mathrm{~K}$ & spherical & $\begin{array}{c}80.6 \pm 0.9 \\
\%\end{array}$ & $\begin{array}{l}4.54 \pm 2.54 \\
\mu \text { mean m } \\
\text { diameter }\end{array}$ & $\begin{array}{c}\text { Soluble } \\
\text { contaminants } \\
\text { only }\end{array}$ & $50 \mathrm{~g} /$ hour \\
\hline SPIPA-B & $\begin{array}{l}\text { ultraso } \\
\text { unds }\end{array}$ & LN2 & $77 \mathrm{~K}$ & spherical & $\begin{array}{c}49.3 \pm 1.4 \\
\%\end{array}$ & $\begin{array}{l}67+/-31 \\
\text { mean } \mu \mathrm{m} \\
\text { diameter }\end{array}$ & $\begin{array}{c}\text { Soluble } \\
\text { contaminants }+ \\
1 \% \text { insoluble } \\
\text { in suspension }\end{array}$ & $200 \mathrm{~g} /$ hour \\
\hline SPIPA-C & $\begin{array}{c}\text { compre } \\
\text { ssor }\end{array}$ & LN2 & $77 \mathrm{~K}$ & spherical & $\begin{array}{c}\text { Not } \\
\text { measured }\end{array}$ & $\begin{array}{l}2 \text { to } 100 \\
\mu \mathrm{m} \\
\text { diameter }\end{array}$ & $\begin{array}{c}\text { Soluble } \\
\text { contaminants }+ \\
\text { up to } 50 \mathrm{wt} . \% \\
\text { insoluble in } \\
\text { suspension }\end{array}$ & $>\mathrm{kg} /$ hour \\
\hline $\begin{array}{c}\text { Frost } \\
\text { cold-trap }\end{array}$ & $\begin{array}{l}\text { Vapour } \\
\text { conden } \\
\text { sation }\end{array}$ & $\begin{array}{l}\text { Onto } \\
\text { the } \\
\text { cold } \\
\text { surface } \\
\text { of the } \\
\text { sample }\end{array}$ & $\begin{array}{c}100-200 \\
K\end{array}$ & $\begin{array}{l}\text { Hemisphe } \\
\text { rical, then } \\
\text { dendritic } \\
\text { with } \\
\text { needles } \\
\text { (Wu et } \\
\text { al., 2017) }\end{array}$ & $\begin{array}{c}\text { Not } \\
\text { measured }\end{array}$ & $\begin{array}{l}\text { From } \\
\mu \text { m's to } \\
\text { millimetri } \\
\text { c needles }\end{array}$ & no & $\begin{array}{l}\text { Variable } \\
\text { upon T of } \\
\text { the } \\
\text { sample, } \\
\text { relative } \\
\text { humidity, } \\
\text { etc. }\end{array}$ \\
\hline
\end{tabular}

Table 4: Parameters and properties of the different ice production setups developed and their products. 


\begin{tabular}{|c|c|c|}
\hline Sample composition /evolution & Measurements & Publication \\
\hline 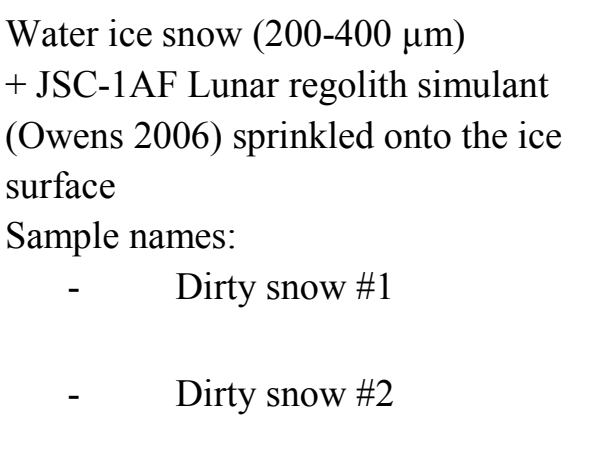 & $\begin{array}{l}\text { Multi-angular } \\
\text { bidirectional } \\
\text { reflectance - } 650 \mathrm{~nm} \\
\text { (70nm FWHM) } \\
\text { Data available in } \\
\text { DACE } \\
\text { https://dace.unige.ch/ }\end{array}$ & Pommerol et al., 2011 \\
\hline $\begin{array}{l}\text { JSC Mars-1 regolith simulant + water } \\
\text { ice } \\
\text { - "frozen A": matrix of ice } \\
\text { between individual grains in the } \\
\text { bulk, grains completely free of ice } \\
\text { at the surface } \\
\text { - "frozen B": very thin ice } \\
\text { film on top of the grains } \\
\text { - "frozen C": thicker film of } \\
\text { water ice filling the gaps between } \\
\text { grains and hiding the topography } \\
\text { of the underlying surface" } \\
\text { - fine frost deposited on the } \\
\text { surface of the grains }\end{array}$ & $\begin{array}{l}\text { Multi-angular } \\
\text { bidirectional } \\
\text { reflectance - } 650 \mathrm{~nm} \\
\text { (70nm FWHM) } \\
\text { Data available in } \\
\text { DACE } \\
\text { https://dace.unige.ch/ }\end{array}$ & Pommerol et al., 2013 \\
\hline $\begin{array}{l}\text { Carbon black }(1 \mathrm{wt} \%)+\mathrm{JSC} 1-\mathrm{AF} \\
\text { lunar regolith simulant }(5 \mathrm{wt} \%)+ \\
\text { SPIPA-A water ice particles } \\
\text { (mixing in liquid nitrogen, inter- } \\
\text { mixture) } \\
\text { Sublimation, formation of a crust of } \\
\text { dust on top of the ice }\end{array}$ & $\begin{array}{l}\text { Hyperspectral } \\
\text { reflectance data from } \\
0.4 \text { to } 2.3 \mu \mathrm{m} \\
\text { Data available in } \\
\text { ByPASS/SSHADE } \\
\text { https://www.sshade.eu/ } \\
\text { db/bypass }\end{array}$ & Pommerol et al., 2015 \\
\hline
\end{tabular}




\begin{tabular}{|c|c|c|}
\hline $\begin{array}{l}\text { JSC1-AF }+10,20,35,50,75 \mathrm{wt} \% \\
\text { SPIPA-A water ice particles ( } 5 \\
\text { samples) } \\
\text { JSC1-AF + 10, 35, 50, } 75 \mathrm{wt} \% \\
\text { SPIPA-B water ice particles ( } 4 \\
\text { samples) }\end{array}$ & $\begin{array}{l}\text { Multi-angular } \\
\text { bidirectional } \\
\text { reflectance } \\
\left(750 \mathrm{~nm}, \mathrm{i}=20^{\circ}, 70^{\circ}\right) \\
\text { Data available in } \\
\text { DACE } \\
\text { https://dace.unige.ch/ }\end{array}$ & Yoldi et al., 2015 \\
\hline $\begin{array}{l}\text { SPIPA-A water ice particles }+0.1 \% \\
\text { tholins inter-mixture } \\
\text { SPIPA-B water ice particles }+0.1 \% \\
\text { tholins inter-mixture } \\
\text { SPIPA-B water ice particles }+0.1 \% \\
\text { tholins intra-mixture } \\
\text { Sublimation, formation of crusts of } \\
\text { dust on top of the ice }\end{array}$ & $\begin{array}{l}\text { Hyperspectral } \\
\text { reflectance data from } \\
0.4 \text { to } 2.3 \mu \mathrm{m} \\
\text { Data available in } \\
\text { ByPASS/SSHADE } \\
\underline{\text { https://www.sshade.eu/ }} \\
\underline{\mathrm{db} / \text { bypass }}\end{array}$ & Poch et al., 2016a \\
\hline $\begin{array}{l}\text { SPIPA-B water ice particles + } \\
\text { - } 0.1 \%, 1 \% \text { forsterite intra-, } \\
\text { inter-mixture ( } 4 \text { samples) } \\
\text { - } 0.1 \%, 1 \% \text { montmorillonite } \\
\text { intra-, inter-mixture ( } 4 \\
\text { samples) } \\
\text { - } 0.1 \% \text { forsterite, } 0.05 \% \text { tholins, } \\
0.01 \% \text { montmorillonite inter-, } \\
\text { intra-mixture ( } 2 \text { samples) } \\
\text { - } 1 \% \text { forsterite, } 0.5 \% \text { tholins, } \\
0.1 \% \text { montmorillonite inter-, } \\
\text { intra-mixture ( } 2 \text { samples) } \\
\text { Sublimation, formation of crusts of } \\
\text { dust on top of the ice }\end{array}$ & $\begin{array}{l}\text { Hyperspectral } \\
\text { reflectance data from } \\
0.4 \text { to } 2.3 \mu \mathrm{m} \\
\text { Data available in } \\
\text { ByPASS/SSHADE } \\
\text { https://www.sshade.eu/ } \\
\underline{\mathrm{db} / \text { bypass }} \\
\text { Multi-angular } \\
\text { bidirectional } \\
\text { reflectance of the } \\
\text { sublimate residue of } \\
\text { montmorillonite } 1 \% \\
\text { intra-mixture } \\
\left(750 \text { nm, i }=0^{\circ}, 60^{\circ}\right) \\
\text { Data available in } \\
\text { DACE } \\
\text { https://dace.unige.ch/ }\end{array}$ & Poch et al., 2016b \\
\hline 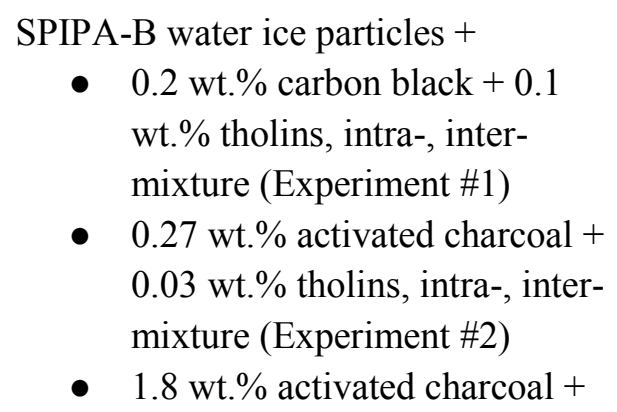 & $\begin{array}{l}\text { Hyperspectral } \\
\text { reflectance data from } \\
0.4 \text { to } 2.3 \mu \mathrm{m} \\
\text { Data available in } \\
\text { ByPASS/SSHADE } \\
\underline{\mathrm{https}: / / \text { www.sshade.eu/ }} \\
\text { db/bypass }\end{array}$ & Jost et al., 2017a \\
\hline
\end{tabular}




\begin{tabular}{|c|c|c|}
\hline $\begin{array}{l}\quad 0.2 \mathrm{wt} . \% \text { tholins, intra-, inter- } \\
\text { mixture (Experiment \#3) } \\
\text { Coarser fraction of intra-mixture ice } \\
\text { particles }(>800 \mu \mathrm{m} \text { ) containing } 1.8 \\
\text { wt. } \% \text { activated charcoal }+0.2 \mathrm{wt} . \% \\
\text { tholins (Experiment \#3) } \\
\text { Sublimation, formation of crusts of } \\
\text { dust on top of the ice }\end{array}$ & $\begin{array}{l}\text { Multi-angular } \\
\text { bidirectional } \\
\text { reflectance } \\
\left(750 \mathrm{~nm}, \mathrm{i}=0^{\circ}, 60^{\circ}\right) \\
\text { Data available in } \\
\text { DACE } \\
\text { https://dace.unige.ch/ }\end{array}$ & \\
\hline $\begin{array}{l}\text { SPIPA-A water ice particles }+0.1 \% \text {, } \\
1 \% \text { tholins, inter-mixture } \\
\text { SPIPA-B water ice particles }+0.1 \% \text {, } \\
1 \% \text { tholins, inter-, intra-mixture } \\
\text { SPIPA-A water ice particles }+5 \% \\
\text { carbon black, inter-mixture } \\
\text { SPIPA-B water ice particles }+2 \% \\
\text { carbon black, inter-mixture } \\
\text { SPIPA-A water ice particles }+10 \% \text {, } \\
20 \%, 33 \%, 43 \%, 50 \% \text { charcoal, inter- } \\
\text { mixture } \\
\text { SPIPA-B water ice particles }+20 \% \text {, } \\
29 \% \text { charcoal, inter-mixture }\end{array}$ & $\begin{array}{l}\text { Multi-angular } \\
\text { bidirectional } \\
\text { reflectance } \\
(450 / 550 / 650 / 750 / 905 / \\
1064 \mathrm{~nm}) \\
\text { Data available in } \\
\text { DACE } \\
\underline{\text { https://dace.unige.ch/ }}\end{array}$ & Jost et al., 2017b \\
\hline $\begin{array}{l}\text { SPIPA-B water ice particles }+ \\
\text { Deinococcus radiodurans }\left(10^{9}, 10^{5}\right. \\
\text { org./mL), intra-mixture } \\
\text { Sublimation, formation of a desiccated } \\
\text { layer of bacteria on top of the ice }\end{array}$ & $\begin{array}{l}\text { Hyperspectral } \\
\text { reflectance data from } \\
0.4 \text { to } 2.3 \mu \mathrm{m} \\
\text { Data available in } \\
\text { ByPASS/SSHADE } \\
\text { https://www.sshade.eu/ } \\
\underline{\text { db/bypass }} \\
\text { Multi-angular } \\
\text { bidirectional } \\
\text { reflectance } \\
\left(450,750 \text { nm, i }=0^{\circ},\right. \\
\left.60^{\circ}\right) \\
\text { Data available in } \\
\text { DACE } \\
\text { https://dace.unige.ch/ }\end{array}$ & Poch et al., 2017 \\
\hline
\end{tabular}

Table 2: Summary of ice-dust mixtures whose spectro-photometric properties were measured in the LOSSy and published 


\begin{tabular}{|c|c|c|c|c|}
\hline Samples & $\begin{array}{c}\text { Dust-ice } \\
\text { volumetric } \\
\text { ratio values }\end{array}$ & $\begin{array}{c}\text { Porosity range } \\
(\%)\end{array}$ & $\begin{array}{c}\text { Temperature } \\
(\mathrm{K})\end{array}$ & $\varepsilon^{\prime}$ 'range \\
\hline $\begin{array}{c}\text { Etna volcanic } \\
\text { ash }\end{array}$ & $/$ & $52-59$ & 298 & $2.90-3.50$ \\
\hline $\begin{array}{c}\text { JSC-1A lunar } \\
\text { simulant }\end{array}$ & $/$ & $48-58$ & 298 & $3.00-3.40$ \\
\hline SPIPA-A & $/$ & $49-86$ & 243 & $1.20-1.90$ \\
\hline SPIPA-B & $/$ & $31-50$ & 243 & $1.70-2.20$ \\
\hline Tholins & $/$ & $70-95$ & 243 & $1.04-1.60$ \\
\hline $\begin{array}{c}\text { Mixture JSC- } \\
\text { 1A/SPIPA-A }\end{array}$ & $0.10,0.31$, & $51-91$ & 243 & $1.10-2.70$ \\
\hline $\begin{array}{c}\text { Mixture } \\
\text { JSC-1A/SPIPA- } \\
\text { B }\end{array}$ & 0.31 & $42-50$ & 243 & $2.00-2.30$ \\
\hline $\begin{array}{c}\text { Mixture } \\
\text { Tholins/SPIPA- } \\
\text { A }\end{array}$ & 0.60 & $50-80$ & 243 & $1.30-2.10$ \\
\hline
\end{tabular}

Table 3: summarizing samples properties and measurements of the real part of the permittivity, $\varepsilon^{\prime}$, in the $0.05-2 \mathrm{GHz}$ range (from Brouet et al., 2015, 2016a, 2016b). 


\begin{tabular}{|c|c|}
\hline Objective & Technical solution \\
\hline $\begin{array}{c}\text { A chamber easier to clean as } \\
\text { large amounts of dust are } \\
\text { regularly emitted and } \\
\text { deposited in various places of } \\
\text { the chamber. }\end{array}$ & $\begin{array}{c}\text { The new chamber will be equipped with a large } \\
\text { door so that the interior can be easily accessed. }\end{array}$ \\
\hline $\begin{array}{c}\text { Controlled temperature at the } \\
\text { base of the sample holder }\end{array}$ & $\begin{array}{c}\text { A plate with a regulated temperature at the base of } \\
\text { the sample holder, using a 1-stage helium cryostat } \\
\text { to reach low temperatures. }\end{array}$ \\
\hline More versatile facility & $\begin{array}{c}\text { An interchangeable upper flange above the sample } \\
\text { to permit the installation of different types of } \\
\text { setups. }\end{array}$ \\
\hline OCTOPUS & $\begin{array}{c}\text { Vertical viewports on the sides of the chamber and } \\
\text { movable mirrors above the sample to observe its } \\
\text { surface from beside the chamber. }\end{array}$ \\
\hline Dielectric & $\begin{array}{c}\text { Holders for optical fibres all around the samples to } \\
\text { illuminate the surface of the samples at various } \\
\text { wavelengths and collect light for optical } \\
\text { measurement devices. }\end{array}$ \\
\hline
\end{tabular}

Table 7: Objectives and technical solutions for the new version of the SCITEAS simulation chamber (SCITEAS-2). 
Electronic Supplementary Materials

\section{Click here to access/download \\ Electronic Supplementary Materials ESM1.docx}

